\title{
QUELQUES ASPECTS DIOPHANTIENS DES VARIÉTÉS TORIQUES PROJECTIVES
}

\author{
par \\ Patrice Philippon \& Martín Sombra
}

An Wolfgang Schmidt

Der oft und schick

'nen Edelsatz

Geschmiedet hat

\begin{abstract}
Résumé. - On présente plusieurs facettes des variétés toriques projectives, intéressantes du point de vue de la géométrie diophantienne. On montre comment la théorie s'explicite sur un certain nombre d'exemples significatifs et on établit également un théorème de type Bézout pour les poids de Chow des variétés projectives.
\end{abstract}

\begin{abstract}
Some diophantine aspects of projective toric varieties. We present several facets of projective toric varieties, of interest from the point of view of Diophantine geometry. We make explicit the theory in a number of meaningful examples and we also prove a Bézout type theorem for Chow weight of projective varieties.
\end{abstract}

\section{Introduction et résultats}

Les variétés toriques jouent un rôle important au carrefour de l'algèbre, la géométrie et la combinatoire. Elles constituent une classe de variétés suffisamment rigide pour que beaucoup des invariants s'explicitent en termes combinatoires, et en même temps suffisamment riche pour permettre de tester et illustrer diverses conjectures et théories abstraites. Elle trouve application dans de nombreuses branches des mathématiques : géométrie algébrique bien sûr, algèbre commutative, combinatoire, calcul formel, géométries symplectique et kählerienne, topologie et physique mathématique, voir par exemple [Ful93, GKZ94, Stu96], Cox01, Aud91, Don02.

Par définition, les variétés toriques projectives sont les compactifications équivariantes des translatés de sous-tores des tores multiplicatifs $\mathbf{G}_{m}^{N}$. Du point de vue de la géométrie diophantienne, ces variétés se trouvent à la croisée des problèmes de Lehmer et de Bogomolov

Classification mathématique par sujets (2000). — Primaire: 11G50; Secondaire: 14M25, $14 \mathrm{G} 40$.

Mots clefs. - Variété torique, hauteur normalisée, multihauteurs, fonction de Hilbert arithmétique, poids de Chow, volume mixte, indice d'obstruction, minimums successifs.

P. Philippon a été partiellement financé par une allocation de recherche de la Fondation Alexander von Humboldt pendant la réalisation de ce travail. M. Sombra a été financé par le Programme Ramón y Cajal du Ministerio de Educación y Ciencia, Espagne. 
généralisés sur les tores. En effet, on sait que lorsqu'elles ne sont pas de torsion, les minorations pour la hauteur normalisée de ces variétés sont de nature fondamentalement arithmétique, dépendant essentiellement du corps de définition de la variété. Au contraire, pour les sousvariétés de $\mathbf{G}_{m}^{N}$ qui ne sont pas des translatées de sous-tores on dispose de minorations ne dépendant que de leur géométrie, voir [AD03, AD04, Dav03. D'un autre côté, R. Ferretti Fer03 a exploité les variétés toriques afin de trouver des exemples concrets de l'extension du théorème du sous-espace aux variétés projectives, qu'il a obtenue avec J.-H. Evertse [EF02].

Dans [PS04] (voir aussi PS05]) nous avons étudié l'un des invariants arithmétiques les plus significatifs des variétés dans le cas torique, à savoir leur hauteur normalisée. Cet invariant est l'analogue arithmétique du degré, il mesure la complexité binaire d'une représentation de la variété et contrôle aussi la distribution des points algébriques de petite hauteur sur la variété. Dans PS04, on a donné une expression explicite pour la hauteur normalisée d'une variété torique et plus généralement pour la multihauteur d'un tore par rapport à plusieurs plongements monomiaux.

Ces résultats sont en parfait parallèle avec la théorie géométrique. En fait, on construit un objet adélique $\Theta_{X}$ associé à une variété torique $X$ (constitué par une famille finie de fonctions concaves et affines par morceaux) qui est le pendant arithmétique du polytope classiquement associé à l'action du tore et dont l'intégrale donne la hauteur. Grâce à cette approche, il est possible de calculer explicitement cette quantité pour n'importe qu'elle variété torique particulière et de tester utilement des conjectures et résultats.

Le présent texte a le double propos d'introduire le lecteur à l'étude des variétés toriques

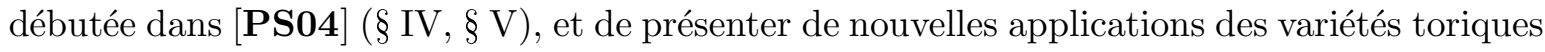
à des problèmes diophantiens ou d'origine diophantienne $(\S$ II $\S$ III $\S$ VI, $\S$ VII).

Dans le $\S \amalg$ on s'intéresse aux indices d'obstruction successifs des variétés toriques définies sur un corps algébriquement clos K. Il s'agit des plus petits degrés de formes d'une suite sécante (soit globalement, soit dans un ouvert) découpant un ensemble algébrique ayant la variété comme composante. Différentes variantes de ces indices jouent un rôle important dans les généralisations des problèmes de Lehmer et de Bogomolov par exemple, voir AD03.

Les sous-variétés toriques de $\mathbf{P}^{N}(\mathbf{K})$ correspondent à des idéaux binomiaux premiers et homogènes de l'anneau $\mathbf{K}\left[x_{0}, \ldots, x_{N}\right]$. De plus, les binômes engendrant l'idéal d'une variété torique $X$ s'explicitent en termes d'un certain $\mathbf{Z}$-module $\Gamma_{X} \subset \mathbf{Z}^{N+1}$ naturellement associé à $X$, voir ES96] ou $\S$ [1]

On montre que le premier indice d'obstruction d'une variété torique $X$ est égal au premier minimum de $\Gamma_{X}$ par rapport à une métrique convenable; et plus généralement, que les indices d'obstruction successifs $\omega_{i}\left(X ;\left(\mathbf{P}^{N}\right)^{\circ}\right)$ de cette variété relatifs à l'ouvert $\left(\mathbf{P}^{N}\right)^{\circ}$ coïncident avec les minimums successifs de $\Gamma_{X}$ et qu'ils se réalisent par des équations binomiales (Proposition (II.4). Via ce résultat, le deuxième théorème de Minkowski se traduit en des estimations pour le produit des indices d'obstruction successifs, qui précisent dans le cas torique les estimations de M. Chardin Cha89 et de Chardin et P. Philippon CP99:

Proposition 0.1. - Soit $X \subset \mathbf{P}^{N}$ une variété torique de dimension n, alors

$$
\operatorname{deg}(X) \leq \omega_{1}\left(X ;\left(\mathbf{P}^{N}\right)^{\circ}\right) \cdots \omega_{N-n}\left(X ;\left(\mathbf{P}^{N}\right)^{\circ}\right) \leq(N+1)^{N-n} \operatorname{deg}(X) .
$$

En outre, le réseau $\Gamma_{X}$ s'identifie au réseau des périodes de l'application exponentielle restreinte à l'espace tangent en l'origine de $X^{\circ}$. On retrouve ainsi au $\S \amalg$ certains résultats de [BP88 reliant degré et multi-degrés d'un sous-groupe algébrique d'un tore multiplicatif au volume de son réseau des périodes et à la hauteur de son espace tangent. 
Dans l'article [PS06 on poursuit une étude approfondie des indices d'obstruction des variétés toriques et de son application à la minoration de la hauteur des points dans ces variétés.

Soit maintenant $X \subset \mathbf{P}^{N}$ une variété quelconque de dimension $n$ et $\tau=\left(\tau_{0}, \ldots, \tau_{N}\right) \in$ $\mathbf{R}^{N+1}$ un vecteur poids. Soient $n+1$ groupes $U_{0}, \ldots, U_{n}$ de $N+1$ variables chacun et considérons la forme de Chow de $X$

$$
C h_{X}=\sum_{a \in \mathbf{N}^{(n+1)(N+1)}} c_{a} U_{0}^{a_{0}} \cdots U_{n}^{a_{n}} \in \mathbf{K}\left[U_{0}, \ldots, U_{n}\right]
$$

Le poids de Chow relatif à $\tau$ (ou $\tau$-poids de Chow) de $X$ est défini comme le poids de sa forme de Chow par rapport au vecteur $(\tau, \ldots, \tau) \in \mathbf{R}^{(n+1)(N+1)}$, c'est-à-dire

$$
e_{\tau}(X):=\max \left\{\left\langle a_{0}, \tau\right\rangle+\cdots+\left\langle a_{n}, \tau\right\rangle: a \in \mathbf{N}^{(n+1)(N+1)} \text { tel que } c_{a} \neq 0\right\},
$$

où $\langle\cdot, \cdot\rangle$ désigne le produit scalaire ordinaire.

Le poids de Chow a été introduit par D. Mumford Mum77 en relation avec la stabilité des variétés projectives. On le retrouve (et en particulier l'énoncé IV.3 du présent texte) dans un travail de S.K. Donaldson Don02 montrant la relation entre stabilité des variétés toriques et existence de métrique kählerienne à courbure constante. Il apparaît également en géométrie diophantienne au travers des travaux de Ferretti Fer03, Evertse et Ferretti [EF02 et dans notre formule pour la hauteur d'une variété torique [PS04.

Pour $\tau \in \mathbf{Z}^{N+1}$, considérons l'action du sous-groupe à un paramètre

$$
*_{\tau}: \mathbf{G}_{m} \times \mathbf{P}^{N} \rightarrow \mathbf{P}^{N}, \quad\left(t,\left(x_{0}: \cdots: x_{N}\right)\right) \mapsto\left(t^{\tau_{0}} x_{0}: \cdots: t^{\tau_{N}} x_{N}\right)
$$

et la déformation torique $X_{\tau}$ de $X$ associée, définie comme l'adhérence de Zariski de l'ensemble

$$
\left\{\left((1: t), t *_{\tau} x\right): t \in \mathbf{G}_{m}, x \in X\right\} \subset \mathbf{P}^{1} \times \mathbf{P}^{N} .
$$

La variété initiale de $X$ relative au poids $\tau \in \mathbf{Z}^{N+1}$ est par définition

$$
\operatorname{init}_{\tau}(X):=\iota^{*}\left(X_{\tau} \cdot\left(\{(0: 1)\} \times \mathbf{P}^{N}\right)\right) \in Z_{n}\left(\mathbf{P}^{N}\right),
$$

où $\iota: \mathbf{P}^{N} \rightarrow \mathbf{P}^{1} \times \mathbf{P}^{N}$ désigne l'inclusion $\left(x_{0}: \cdots: x_{N}\right) \mapsto\left((0: 1),\left(x_{0}: \cdots: x_{N}\right)\right)$. Autrementdit, $\operatorname{init}_{\tau}(X)$ est le cycle limite $\lim _{t \rightarrow \infty} t *_{\tau} X$ de $X$ sous l'action $*_{\tau}$. C'est un cycle de même dimension et degré que $X$.

On montre au $\S \llbracket$ que lorsque $\tau \in \mathbf{N}^{N+1}$, le poids de Chow relatif à $\tau$ s'interprète comme un bi-degré d'une variante de la déformation torique ci-dessus et se comporte donc comme une hauteur. Comme conséquence de cette interprétation, on démontre un théorème de Bézout pour le poids de Chow du cycle intersection $X \cdot H$, qui précise la majoration obtenue par Ferretti [Fer03 Prop. 4.3].

Théorème 0.2. - Soit $X \subset \mathbf{P}^{N}$ une variété projective et $H \in \operatorname{Div}\left(\mathbf{P}^{N}\right)$ un diviseur ne contenant pas $X$, alors pour $\tau \in \mathbf{Z}^{N+1}$ on a

$$
\begin{aligned}
e_{\tau}(X \cdot H)=e_{\tau}(X) \operatorname{deg}(H)+e_{\tau}(H) \operatorname{deg}(X) & -\left(\tau_{0}+\cdots+\tau_{N}\right) \operatorname{deg}(H) \operatorname{deg}(X) \\
& -\sum_{Y \in \operatorname{Irr}_{\left.\operatorname{init}_{\tau}(X)\right)}} m\left(X_{\tau} \cdot H_{\tau} ; \iota(Y)\right) \operatorname{deg}(Y)
\end{aligned}
$$

où la somme porte sur les composantes irréductibles de $\operatorname{init}_{\tau}(X)$.

En particulier, si $H$ est effectif on a pour tout $\tau \in \mathbf{R}^{N+1}$

$$
e_{\tau}(X \cdot H) \leq e_{\tau}(X) \operatorname{deg}(H)+e_{\tau}(H) \operatorname{deg}(X)-\left(\tau_{0}+\cdots+\tau_{N}\right) \operatorname{deg}(H) \operatorname{deg}(X),
$$

avec égalité si et seulement si les variétés initiales de $X$ et $H$ s'intersectent proprement. 
Remarque 0.3. - Lorsque $H$ est effectif, le terme correctif $\sum_{Y} m\left(X_{\tau} \cdot H_{\tau} ; \iota(Y)\right) \operatorname{deg}(Y)$ est le degré la partie du cycle intersection $X_{\tau} \cdot H_{\tau}$, contenue dans la variété initiale de $X$.

Suivant l'attitude générale adoptée dans ce texte nous écrivons également au $\S$ VII ce théorème en termes combinatoires pour l'intersection d'une variété torique avec un diviseur monomial. Comme autre application de la formule de la hauteur d'une variété torique, on en déduit dans ce cas un théorème de Bézout exact pour la hauteur normalisée du cycle intersection (voir Corollaire VII.6).

L'étude des points algébriques de petite hauteur (ou petits points) a reçu une attention considérable au cours des dernières années. La taille des petits points dans une variété est quantifié par ses minimums algébriques successifs.

Soit $X \subset \mathbf{P}^{N}(\overline{\mathbf{Q}})$ une variété quasi-projective quelconque, de dimension $n$. Pour $\theta \geq 0$ on pose $X(\theta)$ pour l'ensemble des points de $X$ de hauteur normalisée $\widehat{h}$ majorée par $\theta$. Pour $i=1, \ldots, n+1$, le $i$-ème minimum algébrique de $X$ par rapport à la hauteur normalisée est

$$
\widehat{\mu}_{i}(X):=\inf \{\theta: \operatorname{dim}(\overline{X(\theta)}) \geq n-i+1\},
$$

où $\overline{X(\theta)}$ désigne l'adhérence de Zariski de $X(\theta)$. On écrit $\widehat{\mu}^{\text {ess }}(X):=\widehat{\mu}_{1}(X)$ et $\widehat{\mu}^{\text {abs }}(X):=$ $\widehat{\mu}_{n+1}(X)$ pour les minimums essentiel et absolu respectivement; on a $\widehat{\mu}_{1}(X) \geq \cdots \geq \widehat{\mu}_{n+1}(X)$ $\geq 0$.

La répartition de la hauteur des points algébriques d'une variété projective fermée $X$ est en relation avec sa hauteur, le lien est donné par le théorème des minimums successifs $\mathbf{Z h a 9 5}$ Thm. 5.2 et Lem. 6.5]:

$$
\widehat{\mu}_{1}(X)+\cdots+\widehat{\mu}_{n+1}(X) \leq \frac{\widehat{h}(X)}{\operatorname{deg}(X)} \leq(n+1) \widehat{\mu}_{1}(X) .
$$

Comme application de la formule pour la hauteur d'une variété torique de [PS04], on construit au $\S 6$ des exemples montrant que toute configuration possible des minimums successifs est arbitrairement approchable et que le quotient $\widehat{h}(X) / \operatorname{deg}(X)$ peut atteindre n'importe quelle valeur dans l'intervalle autorisé par l'encadrement ci-dessus:

Théorème 0.4. - Soient $n, N \in \mathbf{N}$ tels que $N \geq 3 n+1$ et $\mu_{1}, \ldots, \mu_{n+1}, \nu \in \mathbf{R}$ tels que

$$
\mu_{1} \geq \cdots \geq \mu_{n+1} \geq 0 \quad \text { et } \quad \mu_{1}+\cdots+\mu_{n+1} \leq \nu<(n+1) \mu_{1} .
$$

Alors pour $0<\varepsilon_{1} \leq(n+1) \mu_{1}-\nu, \varepsilon_{2}>0$ arbitraires, il existe une variété torique $X \subset \mathbf{P}^{N}$ de dimension $n$ telle que

$$
0<\mu_{i}-\widehat{\mu}_{i}(X) \leq \varepsilon_{1} \quad \text { pour } i=1, \ldots, n+1 \text { et }\left|\frac{\widehat{h}(X)}{\operatorname{deg}(X)}-\nu\right|<\varepsilon_{2} \mu_{1} .
$$

De plus, la variété $X$ peut être choisie de degré $\leq\left(4 n^{2} \varepsilon_{2}^{-1}\right)^{n}$ et définie sur une extension kummerienne $K=\mathbf{Q}\left(2^{1 / \ell}\right)$ de degré $\leq\left\lfloor\log (2) \varepsilon_{1}^{-1}\right\rfloor+1$.

Les exemples construits présentent une codimension minimale $N-n=2 n+1$ de l'ordre de la dimension de la variété produite. La question se pose donc de savoir ce qu'il en est pour les variétés de petite codimension ( $c f$. Proposition VI.3).

Pour faciliter la lecture, nous avons tressé dans le texte plusieurs paragraphes d'introduction aux variétés toriques $(\S$ I) , aux poids de Chow $(\S$ IV] et à la théorie de l'intersection multiprojective $(\S \llbracket$. Les paragraphes $\S \amalg$ à $\S \mathbb{V I}$ doivent pouvoir être lus de manière essentiellement indépendante. Dans les paragraphes 【à IV nous prenons un corps de base $\mathbf{K}$ algébriquement 
clos quelconque ou $\mathbf{C}$ pour le $\S$ III L'arithmétique apparaît à partir de la fin du $\S$ IV où nous posons nos conventions sur les places et valeurs absolues des corps de nombres (Convention IV.4).

\section{Plan de l'article}

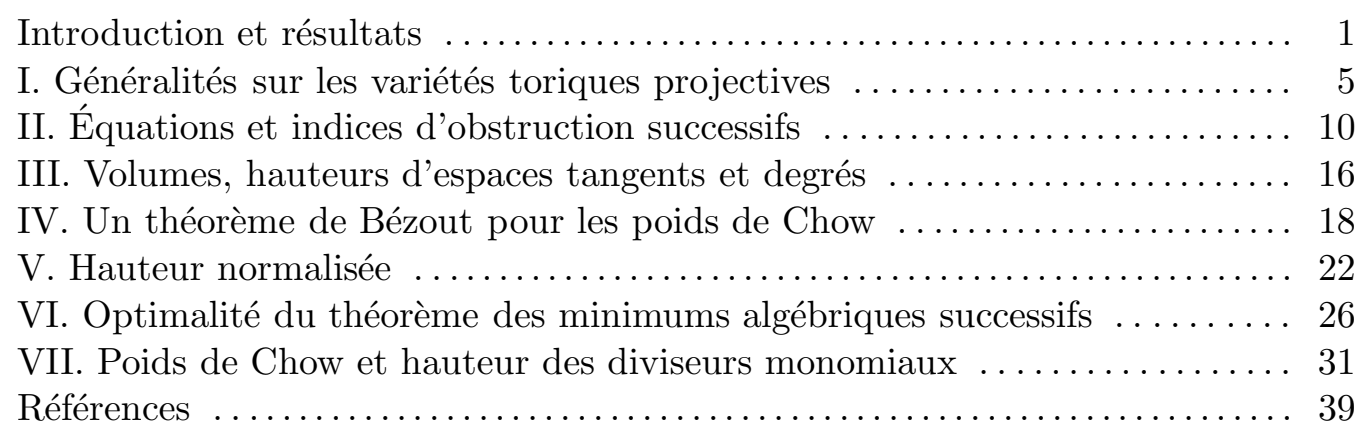

\section{Généralités sur les variétés toriques projectives}

On note $\mathbf{G}_{m}^{n}:=\left(\mathbf{K}^{\times}\right)^{n}$ le tore algébrique et $\mathbf{P}^{N}$ l'espace projectif sur $\mathbf{K}$, de dimension $n$ et $N$ respectivement. Une variété est toujours supposée réduite et irréductible. Pour une famille de polynômes homogènes $f_{1}, \ldots, f_{s} \in \mathbf{K}\left[x_{0}, \ldots, x_{N}\right]$ on pose $Z\left(f_{1}, \ldots, f_{s}\right) \subset \mathbf{P}^{N}$ l'ensemble de ses zéros communs. Réciproquement, pour un ensemble algébrique $Z \subset \mathbf{P}^{N}$ on pose $I(Z)$ son idéal de définition dans $\mathbf{K}\left[x_{0}, \ldots, x_{N}\right]$.

On note $\mathbf{R}_{+}$et $\mathbf{R}_{+}^{\times}$les ensembles des nombres réels non-négatifs et positifs, respectivement. On note encore $\mathbf{N}$ et $\mathbf{N}^{\times}$les entiers naturels avec et sans 0 , respectivement. Pour $N, D \in \mathbf{N}$ on pose $\mathbf{N}_{D}^{N+1}:=\left\{a \in \mathbf{N}^{N+1}: a_{0}+\cdots+a_{N}=D\right\}$.

Dans ce paragraphe on donne un bref aperçu des propriétés géométriques des variétés toriques. Pour plus de détails, on renvoie le lecteur à GKZ94, Ful93, Ewa96, Cox01.

Soit $\mathcal{A}=\left(a_{0}, \ldots, a_{N}\right) \in\left(\mathbf{Z}^{n}\right)^{N+1}$ une suite de $N+1$ vecteurs de $\mathbf{Z}^{n}$, on considère l'action diagonale de $\mathbf{G}_{m}^{n}$ sur $\mathbf{P}^{N}$

$$
*_{\mathcal{A}}: \mathbf{G}_{m}^{n} \times \mathbf{P}^{N} \rightarrow \mathbf{P}^{N} \quad, \quad(s, x) \mapsto\left(s^{a_{0}} x_{0}: \cdots: s^{a_{N}} x_{N}\right) .
$$

On s'intéressera à l'adhérence de Zariski des orbites de cette action; pour un point $\alpha=\left(\alpha_{0}\right.$ : $\left.\cdots: \alpha_{N}\right) \in \mathbf{P}^{N}$ on pose

$$
X_{\mathcal{A}, \alpha}:=\overline{\mathbf{G}_{m}^{n} *_{\mathcal{A}} \alpha} \subset \mathbf{P}^{N}
$$

la variété torique projective associée au couple $(\mathcal{A}, \alpha)$. Autrement-dit, $X_{\mathcal{A}, \alpha}$ est l'adhérence de Zariski de l'image de l'application monomiale

$$
\varphi_{\mathcal{A}, \alpha}:=\left.*_{\mathcal{A}}\right|_{\alpha}: \mathbf{G}_{m}^{n} \rightarrow \mathbf{P}^{N}, \quad s \mapsto\left(\alpha_{0} s^{a_{0}}: \cdots: \alpha_{N} s^{a_{N}}\right)
$$

C'est une variété torique projective au sens de GKZ94, c'est-à-dire une sous-variété de $\mathbf{P}^{N}$ stable sous l'action d'un tore $\mathbf{G}_{m}^{n}$, avec une orbite dense $X_{\mathcal{A}, \alpha}^{\circ}:=\mathbf{G}_{m}^{n} *_{\mathcal{A}} \alpha$.

Lorsque le point $\alpha$ est contenu dans un des sous-espaces standard de $\mathbf{P}^{N}$, la sous-variété $X_{\mathcal{A}, \alpha}$ toute entière reste dans cet espace, puisque l'action $*_{\mathcal{A}}$ est diagonale. Quitte à se restreindre au sous-espace standard minimal contenant $\alpha$, on peut supposer sans perte de 
généralité $\alpha \in\left(\mathbf{P}^{N}\right)^{\circ}:=\mathbf{P}^{N} \backslash\left\{x_{0} \cdots x_{N}=0\right\}$ et on fixe désormais pour $\alpha$ des coordonnées $\left(\alpha_{0}, \ldots, \alpha_{N}\right) \in\left(\mathbf{K}^{\times}\right)^{N+1}$.

On notera $X_{\mathcal{A}}$ la variété torique associée à $\mathcal{A} \in\left(\mathbf{Z}^{n}\right)^{N+1}$ et $(1, \ldots, 1) \in\left(\mathbf{K}^{\times}\right)^{N+1}$. Dans ce cas, l'orbite principale $X_{\mathcal{A}}^{\circ}$ est un sous-tore du tore $\mathbf{G}_{m}^{N} \cong\left(\mathbf{P}^{N}\right)^{\circ}$ et en fait tous les sousgroupes connexes de $\left(\mathbf{P}^{N}\right)^{\circ}$ sont de cette forme. Dans le cas général

$$
X_{\mathcal{A}, \alpha}^{\circ}=\alpha \cdot X_{\mathcal{A}}^{\circ}
$$

où · désigne la multiplication dans $\left(\mathbf{P}^{N}\right)^{\circ}$, c'est-à-dire que l'orbite principale de $X_{\mathcal{A}, \alpha}$ est le translaté d'un sous-tore.

Le couple $(\mathcal{A}, \alpha)$ peut s'interpréter comme une suite finie de monômes $\alpha_{0} s^{a_{0}}, \ldots, \alpha_{N} s^{a_{N}}$ de l'anneau des polynômes de Laurent $\mathbf{K}\left[s_{1}^{ \pm 1}, \ldots, s_{n}^{ \pm 1}\right]$, on dit que les $a_{i}$ sont les exposants et les $\alpha_{i}$ les coefficients. Le support

$$
\operatorname{Supp}(\mathcal{A}):=\left\{a_{i_{0}}, \ldots, a_{i_{M}}\right\} \subset \mathbf{Z}^{n}
$$

est l'ensemble des exposants distincts dans $\mathcal{A}$. Les variétés $X_{\mathcal{A}, \alpha}$ et $X_{\operatorname{Supp}(\mathcal{A})}$ sont linéairement isomorphes par l'application

$$
\mathbf{P}^{N} \rightarrow \mathbf{P}^{M}, \quad\left(x_{0}: \cdots: x_{N}\right) \mapsto\left(\alpha_{i_{0}}^{-1} x_{i_{0}}: \cdots: \alpha_{i_{M}}^{-1} x_{i_{M}}\right)
$$

et donc leurs propriétés géométriques sont les mêmes. Pour ces propriétés, on peut donc se ramener au cas habituel où les $a_{i}$ sont tous distincts et $\alpha_{i}=1$ pour tout $i$. Soit $L_{\mathcal{A}} \subset \mathbf{Z}^{n}$ le sous-module engendré par les différences des vecteurs $a_{0}, \ldots, a_{N}$, on a par exemple

$$
\operatorname{dim}\left(X_{\mathcal{A}, \alpha}\right)=\operatorname{rang}_{\mathbf{Z}}\left(L_{\operatorname{Supp}(\mathcal{A})}\right)=\operatorname{rang}_{\mathbf{Z}}\left(L_{\mathcal{A}}\right) .
$$

On introduit encore le polytope $Q_{\mathcal{A}} \subset \mathbf{R}^{n}$ enveloppe convexe des vecteurs $a_{0}, \ldots, a_{N}$.

Lemme I.1. - Soient $\mathcal{A}=\left\{a_{0}, \ldots, a_{N}\right\}$ et $\mathcal{B}=\left\{b_{0}, \ldots, b_{M}\right\} \subset \mathbf{Z}^{n}$ des ensembles de cardinal $N+1$ et $M+1$ respectivement, avec $N \leq M$. Posons $\pi: \mathbf{P}^{M} \rightarrow \mathbf{P}^{N}$ la projection linéaire standard $\left(y_{0}: \cdots: y_{M}\right) \mapsto\left(y_{0}: \cdots: y_{N}\right)$, on a

(a) si $\mathcal{A} \subset \mathcal{B}$ alors $X_{\mathcal{A}}=\overline{\pi\left(X_{\mathcal{B}}\right)}$;

(b) si $Q_{\mathcal{A}}=Q_{\mathcal{B}}$ alors $\pi$ est un morphisme régulier et fini (au sens des fibres), de degré $\operatorname{deg}(\pi)=\left[L_{\mathcal{B}}: L_{\mathcal{A}}\right]$. Si de plus $L_{\mathcal{A}}=L_{\mathcal{B}}$ alors $\pi$ est un isomorphisme entre $X_{\mathcal{B}}^{\circ}$ et $X_{\mathcal{A}}^{\circ}$;

(c) si $Q_{\mathcal{A}}=Q_{\mathcal{B}}=Q$ et si pour toute face $F$ de $Q$ on a $L_{\mathcal{A} \cap F}=L_{\mathcal{B} \cap F}$, alors $\pi: X_{\mathcal{B}} \rightarrow X_{\mathcal{A}}$ est un isomorphisme.

Démonstration. - La partie (a) est conséquence directe des définitions. Pour les parties (b) et (c), l'hypothèse $Q_{\mathcal{A}}=Q_{\mathcal{B}}=Q$ entraîne que la projection $\pi$ est compatible avec la décomposition en orbites (1.3) ci-dessous. Pour chaque face $F$ de $Q, \pi: X_{\mathcal{B}, F}^{\circ} \rightarrow X_{\mathcal{A}, F}^{\circ}$ est une application monomiale de degré $\left[L_{\mathcal{B} \cap F}: \pi^{*}\left(L_{\mathcal{A} \cap F}\right)\right]=\left[L_{\mathcal{B} \cap F}: L_{\mathcal{A} \cap F}\right]$, en particulier on voit que $\pi$ est régulière à fibres finies (et de degré 1 entre $X_{\mathcal{A}}^{\circ}$ et $X_{\mathcal{B}}^{\circ}$ si $L_{\mathcal{A}}=L_{\mathcal{B}}$ ).

Dans (c), l'hypothèse $L_{\mathcal{B} \cap F}=L_{\mathcal{A} \cap F}$ entraîne déjà que $\pi$ est une bijection. Pour chaque exposant $b_{i} \in \mathcal{B}$ considérons la face $F$ de $Q$ de dimension minimale contenant $b_{i}$. On écrit alors $b_{i}=\sum_{j: a_{j} \in \mathcal{A} \cap F} \lambda_{i, j} a_{j}$ avec $\lambda_{i, j} \in \mathbf{Z}, \sum_{j} \lambda_{i, j}=0$. L'inverse est alors $X_{\mathcal{A}} \rightarrow X_{\mathcal{B}}$, $x \mapsto\left(x^{\lambda_{0}}: \cdots: x^{\lambda_{M}}\right)$.

Suivant la philosophie générale, les propriétés géométriques des variétés toriques se traduisent en des énoncés combinatoires sur les vecteurs $a_{0}, \ldots, a_{N} \in \mathbf{Z}^{n}$ définissant l'action. En ce qui concerne la théorie de l'intersection géométrique de ces variétés, le résultat le plus fondamental est que le degré s'identifie au volume du polytope $Q_{\mathcal{A}}$. 
Le sous-module $L_{\mathcal{A}}$ est un réseau de l'espace linéaire $L_{\mathcal{A}} \otimes_{\mathbf{Z}} \mathbf{R} \subset \mathbf{R}^{n}$; on considère la forme volume $\mu_{\mathcal{A}}$ sur cet espace linéaire, invariante par translations et normalisée de sorte que

$$
\mu_{\mathcal{A}}\left(L_{\mathcal{A}} \otimes \mathbf{z} \mathbf{R} / L_{\mathcal{A}}\right)=1 ;
$$

autrement-dit, de sorte que le volume d'un domaine fondamental soit 1. Soit $r:=\operatorname{rang}_{\mathbf{Z}}\left(L_{\mathcal{A}}\right)$, le degré de $X_{\mathcal{A}, \alpha}$ s'explicite comme $r$ ! fois le volume normalisé du polytope associé GKZ94, $\S$ I.6, Thm. 2.3], Ful93, p. 111]

$$
\operatorname{deg}\left(X_{\mathcal{A}, \alpha}\right)=r ! \mu_{\mathcal{A}}\left(Q_{\mathcal{A}}\right) .
$$

Soit maintenant $\eta: \mathbf{Z}^{r} \hookrightarrow \mathbf{Z}^{n}$ une application linéaire injective telle que $\eta\left(\mathbf{Z}^{r}\right)=L_{\mathcal{A}}$ et posons $b_{i}:=\eta^{-1}\left(a_{i}\right) \in \mathbf{Z}^{r}$ puis $\mathcal{B}:=\left(b_{0}, \ldots, b_{N}\right) \in\left(\mathbf{Z}^{r}\right)^{N+1}$, alors $X_{\mathcal{B}, \alpha}=X_{\mathcal{A}, \alpha}$ GKZ94, Ch. 5, Prop. 1.2], et ainsi on peut toujours supposer sans perte de généralité $L_{\mathcal{A}}=\mathbf{Z}^{n}$. Dans cette situation, la forme volume $\mu_{\mathcal{A}}$ coïncide avec la forme volume euclidienne $\operatorname{Vol}_{n}$ sur $\mathbf{R}^{n}$ et donc

$$
\operatorname{dim}\left(X_{\mathcal{A}, \alpha}\right)=n, \quad \operatorname{deg}\left(X_{\mathcal{A}, \alpha}\right)=n ! \operatorname{Vol}_{n}\left(Q_{\mathcal{A}}\right)
$$

Plus généralement, les multidegrés du tore $\mathbf{G}_{m}^{n}$ plongé dans un produit d'espaces projectifs via plusieurs applications monomiales s'expriment comme volumes mixtes des polytopes associés à ces applications. Nous explicitons cela maintenant.

Soit $Z \subset \mathbf{P}^{N_{0}} \times \cdots \times \mathbf{P}^{N_{m}}$ une sous-variété de dimension $n$ et $c=\left(c_{0}, \ldots, c_{m}\right) \in \mathbf{N}_{n}^{m+1}$ avec $0 \leq c_{i} \leq N_{i}$, le multidegré de $Z$ d'indice $c$ est défini par

$$
\operatorname{deg}_{c}(Z):=\operatorname{Card}\left(X \cap \pi_{0}^{-1}\left(E_{0}\right) \cap \cdots \cap \pi_{m}^{-1}\left(E_{m}\right)\right)
$$

où $\pi_{i}$ désigne la projection $\mathbf{P}^{N_{0}} \times \cdots \times \mathbf{P}^{N_{m}} \rightarrow \mathbf{P}^{N_{i}}$ et $E_{i}$ est un sous-espace linéaire générique de $\mathbf{P}^{N_{i}}$ de codimension $c_{i}$.

Pour $i=0, \ldots, m$ on fixe un groupe $x_{i}=\left\{x_{i, 0}, \ldots, x_{i, N_{i}}\right\}$ de $N_{i}+1$ variables chacun et on considère l'anneau $\mathbf{K}\left[x_{0}, \ldots, x_{m}\right]$, multigradué $\operatorname{par} \operatorname{deg}\left(x_{i, j}\right):=e_{i}$ où $e_{0}, \ldots, e_{m} \in \mathbf{Z}^{m+1}$ désignent les vecteurs de la base standard. Ainsi $I(Z) \subset \mathbf{K}\left[x_{0}, \ldots, x_{m}\right]$ est un idéal multihomogène de rang $N_{0}+\cdots+N_{m}-n$. Soient $d_{0}, \ldots, d_{n} \in \mathbf{N}^{m+1}$; pour chaque $d_{i} \in \mathbf{N}^{m+1}$ on introduit un groupe de variables $U_{i}=\left\{U_{i, 0}, \ldots, U_{i, M_{i}}\right\}, M_{i}+1=\prod_{j=0}^{m}\left(\begin{array}{c}d_{j}+N_{j} \\ N_{j}\end{array}\right)$, et on considère la forme résultante d'indice $d_{0}, \ldots, d_{n}$ de $I(Z)$ :

$$
\text { rés }_{d_{0}, \ldots, d_{n}}(I(Z)) \in \mathbf{K}\left[U_{0}, \ldots, U_{n}\right] \text {. }
$$

On renvoie le lecteur à [Rem01a $\S 3]$ ou encore à [PS04, § I.2] pour la définition et propriétés de base des formes résultantes. Maintenant, à un vecteur $c=\left(c_{0}, \ldots, c_{m}\right) \in \mathbf{N}_{n}^{m+1}$ comme ci-dessus on associe l'indice partiel

$$
d(c):=(\underbrace{e_{0}, \ldots, e_{0}}_{c_{0} \text { fois }}, \ldots, \underbrace{e_{m}, \ldots, e_{m}}_{c_{m} \text { fois }}) \in\left(\mathbf{N}^{m+1}\right)^{n},
$$

où $e_{0}, \ldots, e_{m}$ désignent les vecteurs de la base standard de $\mathbf{R}^{m+1}$. Pour toute choix de $d_{0} \in \mathbf{N}^{m+1} \backslash\{\mathbf{0}\}$ on a Rem01a Prop. 3.4 et 2.11]

$$
\operatorname{deg}_{c}(Z)=\operatorname{deg}_{U_{0}}\left(\operatorname{rés}_{d_{0}, d(c)}(I(Z))\right) .
$$

Soit encore $D=\left(D_{0}, \ldots, D_{m}\right) \in\left(\mathbf{N}^{\times}\right)^{m+1}$ et considérons

$$
\begin{aligned}
& \Psi_{D}: \quad \mathbf{P}^{N_{0}} \times \cdots \times \mathbf{P}^{N_{m}} \longrightarrow \mathbf{P}^{\left(\begin{array}{c}
D_{0}+N_{0} \\
N_{0}
\end{array}\right) \cdots\left(\begin{array}{c}
D_{m}+N_{m} \\
N_{m}
\end{array}\right)-1} \\
& \left(x_{0}, \ldots, x_{m}\right) \quad \longmapsto\left(x_{0}^{b_{0}} \cdots x_{m}^{b_{m}}: b_{0} \in \mathbf{N}_{D_{0}}^{N_{0}+1}, \ldots, b_{m} \in \mathbf{N}_{D_{m}}^{N_{m}+1}\right)
\end{aligned}
$$


le plongement mixte associé (composition des plongements de Veronese et Segre), on sait que Rem01b § 2.3]

$$
\operatorname{deg}\left(\Psi_{D}(Z)\right)=\sum_{c \in \mathbf{N}_{n}^{m+1}}\left(\begin{array}{l}
n \\
c
\end{array}\right) \operatorname{deg}_{c}(Z) D^{c} .
$$

Considérons maintenant des ensembles convexes $Q_{1}, \ldots, Q_{n} \subset \mathbf{R}^{n}$, leur volume mixte (ou multi-volume) est défini par la formule de type inclusion-exclusion

$$
\operatorname{MV}\left(Q_{1}, \ldots, Q_{n}\right):=\sum_{j=1}^{n}(-1)^{n-j} \sum_{1 \leq i_{1}<\cdots<i_{j} \leq n} \operatorname{Vol}_{n}\left(Q_{i_{1}}+\cdots+Q_{i_{j}}\right) .
$$

Cette notion généralise le volume d'un ensemble convexe car $\operatorname{MV}(Q, \ldots, Q)=n$ ! $\operatorname{Vol}_{n}(Q)$. Le volume mixte est positif ou nul, symétrique et linéaire en chaque variable $Q_{i}$ par rapport à la somme de Minkowski. On renvoie à [CLO98, § 7.4] ou [Ewa96 pour ses propriétés de base.

Interprétons ces notions dans le cas torique: soient $\mathcal{A}_{0} \in\left(\mathbf{Z}^{n}\right)^{N_{0}+1}, \ldots, \mathcal{A}_{m} \in\left(\mathbf{Z}^{n}\right)^{N_{m}+1}$ tels que $L_{\mathcal{A}_{0}}+\cdots+L_{\mathcal{A}_{m}}=\mathbf{Z}^{n}$. Posons $\underline{\mathcal{A}}:=\left(\mathcal{A}_{0}, \ldots, \mathcal{A}_{m}\right)$ et considérons l'action associée $*_{\underline{\mathcal{A}}}$ de $\mathbf{G}_{m}^{n}$ sur le produit d'espaces projectifs $\mathbf{P}^{N_{0}} \times \cdots \times \mathbf{P}^{N_{m}}$ :

$$
\text { * } \underline{\underline{A}}: \begin{aligned}
\mathbf{G}_{m}^{n} \times \mathbf{P}^{N_{0}} \times \cdots \times \mathbf{P}^{N_{m}} & \longrightarrow \mathbf{P}^{N_{0}} \times \cdots \times \mathbf{P}^{N_{m}} \\
\left(s, x_{0}, \ldots, x_{m}\right) & \longmapsto\left(s * \mathcal{A}_{0} x_{0}, \ldots, s * \mathcal{A}_{m} x_{m}\right) .
\end{aligned}
$$

On considère la variété torique multi-projective $X_{\underline{\mathcal{A}}} \subset \mathbf{P}^{N_{0}} \times \cdots \times \mathbf{P}^{N_{m}}$, adhérence de Zariski de l'orbite du point $((1: \cdots: 1), \ldots,(1: \cdots: 1))$, et plus généralement on pose $X_{\underline{\mathcal{A}}, \underline{\alpha}}$ pour l'adhérence de l'orbite de $\underline{\alpha}=\left(\alpha_{0}, \ldots, \alpha_{m}\right) \in \mathbf{P}^{N_{0}} \times \cdots \times \mathbf{P}^{N_{m}}$.

Proposition I.2. - Soit $c \in \mathbf{N}_{n}^{m+1}$, dans la situation ci-dessus on a

$$
\operatorname{deg}_{c}\left(X_{\underline{\mathcal{A}}}\right)=\operatorname{MV}_{n}(\underbrace{Q_{\mathcal{A}_{0}}, \ldots, Q_{\mathcal{A}_{0}}}_{c_{0} \text { fois }}, \ldots, \underbrace{Q_{\mathcal{A}_{m}}, \ldots, Q_{\mathcal{A}_{m}}}_{c_{m} \text { fois }}) \text {. }
$$

Démonstration. - Pour $D=\left(D_{0}, \ldots, D_{m}\right) \in\left(\mathbf{N}^{\times}\right)^{m+1}$ on pose $D \cdot \underline{\mathcal{A}}:=D_{0} \mathcal{A}_{0}+\cdots+D_{m} \mathcal{A}_{m}$ l'ensemble des sommes de $D_{i}$ éléments pris dans $\mathcal{A}_{i}$ pour $i=0, \ldots, m$ et $Q_{D \cdot \mathcal{A}}=D_{0} Q_{\mathcal{A}_{0}}+\cdots+$ $D_{m} Q_{\mathcal{A}_{m}}$ l'enveloppe convexe de $D \cdot \underline{\mathcal{A}}$. On vérifie $\Psi_{D}\left(X_{\underline{\mathcal{A}}}\right)=X_{D \cdot \underline{\mathcal{A}}}$, et par la multilinéarité du volume mixte

$$
n ! \operatorname{Vol}_{n}\left(Q_{D \cdot \underline{\mathcal{A}}}\right)=\sum_{c \in \mathbf{N}_{n}^{m+1}}\left(\begin{array}{l}
n \\
c
\end{array}\right) \operatorname{MV}_{n}(\underbrace{Q_{\mathcal{A}_{0}}, \ldots, Q_{\mathcal{A}_{0}}}_{c_{0} \text { fois }}, \ldots, \underbrace{Q_{\mathcal{A}_{m}}, \ldots, Q_{\mathcal{A}_{m}}}_{c_{m} \text { fois }}) D^{c} .
$$

On a $\operatorname{deg}\left(\Psi_{D}\left(X_{\underline{\mathcal{A}}}\right)\right)=n ! \operatorname{Vol}_{n}\left(Q_{D \cdot \underline{\mathcal{A}}}\right)$ pour tout $D$ et la comparaison de l'identité ci-dessus avec (I.2) permet de conclure.

Les orbites de l'action $*_{\mathcal{A}}$ sur $X_{\mathcal{A}, \alpha}$ sont en correspondance avec l'ensemble $\mathrm{F}\left(Q_{\mathcal{A}}\right)$ des faces du polytope $Q_{\mathcal{A}}$. Pour chaque face $P$ on associe un point $\alpha_{P}:=\left(\alpha_{P, 0}: \cdots: \alpha_{P, N}\right) \in \mathbf{P}^{N}$ défini par $\alpha_{P, j}:=\alpha_{j}$ si $a_{j} \in P$ et $\alpha_{P, j}:=0$ sinon; la bijection est donnée par [GKZ94, Ch. 5, Prop. 1.9], Ful93, § 3.1]

$$
P \mapsto X_{\mathcal{A}, \alpha, P}^{\circ}:=\mathbf{G}_{m}^{n} *_{\mathcal{A}} \alpha_{P} \subset \mathbf{P}^{N}
$$

On a la décomposition

$$
X_{\mathcal{A}, \alpha}=\bigsqcup_{P \in \mathrm{F}\left(Q_{\mathcal{A}}\right)} X_{\mathcal{A}, \alpha, P}^{\circ}
$$


Posons $N(P):=\operatorname{Card}\left\{i: a_{i} \in P\right\}-1$ et

$$
\mathcal{A}(P)=\left(a_{i}: a_{i} \in P\right) \in\left(\mathbf{Z}^{n}\right)^{N(P)+1}, \quad \alpha(P):=\left(\alpha_{i}: a_{i} \in P\right) \in\left(\mathbf{K}^{\times}\right)^{N(P)+1},
$$

on vérifie que $X_{\mathcal{A}, \alpha, P}^{\circ} \subset \mathbf{P}^{N}$ est l'orbite principale d'une variété torique contenue dans un sous-espace standard de dimension $N(P)$. Restreinte à ce sous-espace, elle s'identifie à la sous-variété $X_{\mathcal{A}(P), \alpha(P)}^{\circ} \subset \mathbf{P}^{N(P)}$, de dimension égale à la dimension (réelle) de la face $P$.

Plus généralement, soit $X_{\underline{\mathcal{A}}} \subset \mathbf{P}^{N_{0}} \times \cdots \times \mathbf{P}^{N_{m}}$ une variété torique multi-projective et posons $Q_{\underline{\mathcal{A}}}:=\left(Q_{\mathcal{A}_{0}}, \ldots, Q_{\mathcal{A}_{m}}\right)$ la famille de $m+1$ polytopes associée.

Pour un polytope $Q \subset \mathbf{R}^{n}$ et un vecteur $b \in \mathbf{R}^{n}$ on note $Q(b)$ la face de $Q$ définie comme l'ensemble des points $u \in Q$ maximisant la fonctionnelle linéaire $u \mapsto\langle b, u\rangle$. Considérons les combinaisons des faces des $Q_{\mathcal{A}_{i}}$ obtenues par cette méthode:

$$
\mathrm{F}\left(Q_{\underline{\mathcal{A}}}\right):=\left\{\left(Q_{\mathcal{A}_{0}}(b), \ldots, Q_{\mathcal{A}_{m}}(b)\right): b \in \mathbf{R}^{n}\right\} \subset \mathrm{F}\left(Q_{\mathcal{A}_{0}}\right) \times \cdots \times \mathrm{F}\left(Q_{\mathcal{A}_{m}}\right) .
$$

On peut vérifier que l'ensemble des sommes de Minkowski $Q_{\mathcal{A}_{0}}(b)+\cdots+Q_{\mathcal{A}_{m}}(b)$ pour $b \in \mathbf{R}^{n}$, coïncide avec l'ensemble des faces de la somme de Minkowski $Q_{\mathcal{A}_{0}}+\cdots+Q_{\mathcal{A}_{m}}$.

Soit $\underline{\alpha}=\left(\alpha_{0}, \ldots, \alpha_{m}\right) \in \mathbf{P}^{N_{0}} \times \cdots \times \mathbf{P}^{N_{m}}$, les orbites de l'action $*_{\underline{\mathcal{A}}}$ sur $X_{\underline{\mathcal{A}}, \underline{\alpha}}$ sont en correspondance avec l'ensemble $\mathrm{F}\left(Q_{\underline{\mathcal{A}}}\right)$. Pour chaque $\underline{P}=\left(P_{0}, \ldots, P_{m}\right) \in \overline{\mathrm{F}}\left(Q_{\underline{\mathcal{A}}}\right)$ on considère le point $\alpha_{\underline{P}}:=\left(\alpha_{P_{0}}, \ldots, \alpha_{P_{m}}\right) \in \mathbf{P}^{N_{0}} \times \cdots \times \mathbf{P}^{N_{m}}$; la bijection est donnée par

$$
\underline{P} \mapsto X_{\underline{\mathcal{A}}, \underline{\alpha}, \underline{P}}^{\circ}:=\mathbf{G}_{m}^{n} *_{\underline{\mathcal{A}}} \alpha_{\underline{P}} \subset \mathbf{P}^{N_{0}} \times \cdots \times \mathbf{P}^{N_{m}},
$$

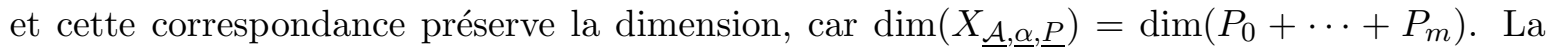
démonstration est une légère variante de [GKZ94, Ch. 5, Prop. 1.9].

Classiquement les variétés toriques sont construites à partir d'éventails, voir par exemple Ful93. Rappelons qu'un éventail est un ensemble de cônes simpliciaux de sommet l'origine engendrés par un nombre fini de points de $\mathbf{Z}^{n}$, tels que toute face et toute intersection de cônes de l'éventail appartienne encore à l'éventail. Le lien avec notre présentation des variétés toriques via des polytopes résulte de ce qu'un polytope $Q$ à sommets dans $\mathbf{Z}^{n}$ détermine un éventail et donc aussi une variété torique au sens classique, qui de plus est munie d'un fibré en droites ample. Dans les alinéa suivants (tirés essentiellement de Cox01 et Ful93]) on explicite cette construction.

Supposons sans perte de généralité $\operatorname{dim}(Q)=n$ et posons $\mathrm{F}_{i}(Q)$ l'ensemble des faces de $Q$ de dimension $i$, de sorte que $\mathrm{F}(Q):=\sqcup_{i=0}^{n} \mathrm{~F}_{i}(Q)$. Pour chaque hyperface (c'est-à-dire face de codimension 1) $F$ de $Q$ on prends le vecteur normal intérieur primitif $v_{F} \in \mathbf{Z}^{n}$ et on note

$$
m_{F}:=-\min \left\{\left\langle x, v_{F}\right\rangle: x \in Q\right\} \in \mathbf{Z},
$$

où $\langle\cdot, \cdot\rangle$ désigne le produit scalaire ordinaire sur $\mathbf{R}^{n}$. On peut donc décrire $Q$ comme intersection de demi-espaces

$$
Q=\bigcap_{F \in \mathrm{F}_{n-1}(Q)}\left\{x \in \mathbf{R}^{n}:\left\langle x, v_{F}\right\rangle \geq-m_{F}\right\}
$$

À chaque face $P$ de $Q$ on associe le cône $\sigma_{P}$ engendré par les vecteurs $v_{F}$ correspondant aux hyperfaces $F \supset P$; on a $\operatorname{dim}(P)+\operatorname{dim}\left(\sigma_{P}\right)=n$. L'ensemble de ces cônes forme un éventail complet $\Sigma$ de $\mathbf{R}^{n}$, c'est-à-dire un éventail recouvrant $\mathbf{R}^{n}$.

La figure suivante montre la correspondance $P \mapsto \sigma_{P}$ entre faces de $Q$ et cônes de $\Sigma$, pour $Q$ le simplexe standard de $\mathbf{R}^{2}$. 


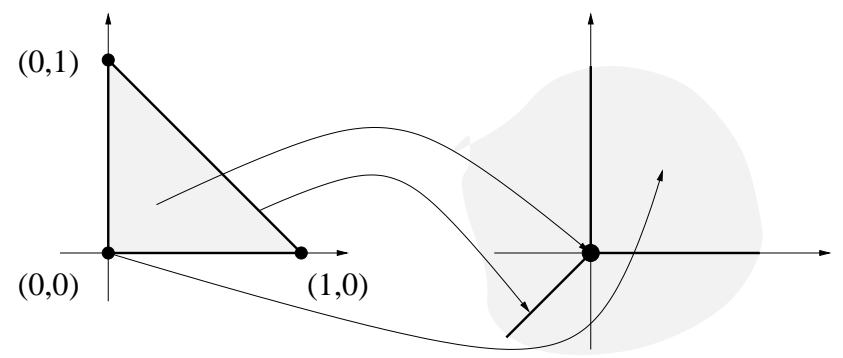

Notons $\mathcal{X}_{\Sigma}$ la variété torique (non plongée dans un espace projectif) définie par cet éventail. Pour chaque hyperface $F$ de $Q$ on note $D_{F}$ le diviseur correspondant à la sous-variété torique de $\mathcal{X}_{\Sigma}$ associée à l'arête $\sigma_{F}$ de $\Sigma$ et on introduit un diviseur de Weil sur $\mathcal{X}_{\Sigma}$ invariant sous l'action de $\mathbf{G}_{m}^{n}$

$$
D_{Q}:=\sum_{F \in \mathrm{F}_{n-1}(Q)} m_{F} D_{F}
$$

qui est en fait un diviseur de Cartier ample [Ful93 § 3.4]. Pour $a \in \mathbf{Z}^{n}$, l'application monomiale $\chi^{a}: \mathbf{G}_{m}^{n} \rightarrow \mathbf{G}_{m}, s \mapsto s^{a}$ peut se voir comme une fonction rationnelle sur $\mathcal{X}_{\Sigma}$. Cette fonction rationnelle s'étend en une section globale de $\mathcal{O}\left(D_{Q}\right)$ si et seulement si $a \in Q$, et en fait

$$
\Gamma\left(\mathcal{X}_{\Sigma}, \mathcal{O}\left(D_{Q}\right)\right)=\bigoplus_{a \in Q \cap \mathbf{Z}^{n}} \mathbf{K} \cdot \chi^{a}
$$

En posant finalement $\mathcal{A}_{Q}:=Q \cap \mathbf{Z}^{n}=\left\{a_{0}, \ldots, a_{N}\right\}$, on vérifie que l'application $\mathbf{G}_{m}^{n} \rightarrow$ $\mathbf{P}^{N}, s \mapsto\left(s^{a_{0}}, \ldots, s^{a_{N}}\right)$ s'étend en un morphisme régulier $\mathcal{X}_{\Sigma} \rightarrow \mathbf{P}^{N}$, dont l'image est la variété torique projective $X_{\mathcal{A}_{Q}}$. De fait, ceci est le morphisme de normalisation de $X_{\mathcal{A}_{Q}}$ Stu96. Cor. 13.6].

Cependant, notons que la notion d'éventail permet de définir des variétés toriques qui, même complètes, n'admettent pas nécessairement de diviseur $\mathbf{G}_{m}^{n}$-invariant ample. Toutefois, lorsqu'une variété torique abstraite possède un tel diviseur, elle admet une application monomiale régulière vers un espace projectif et son image est une variété torique projective du type que nous étudions ici. On notera encore que les variétés toriques abstraites sont des variétés normales, voir [Ful93 § 2.1, p. 29], ce qui n'est pas toujours le cas pour les variétés toriques projectives.

\section{II. Équations et indices d'obstruction successifs}

Soit $X \subset \mathbf{P}^{N}$ une variété projective de dimension $n \geq 0$ et $I(X) \subset \mathbf{K}\left[x_{0}, \ldots, x_{N}\right]$ son idéal de définition, l'indice d'obstruction $\omega(X)$ est défini comme le plus petit degré d'une équation homogène $f \in I(X) \backslash\{0\}$. Plus généralement, pour $i=1, \ldots, N-n$ on définit le $i$-ème indice d'obstruction de $X$ par

$$
\omega_{i}(X):=\min \left\{D \in \mathbf{N}: \operatorname{dim}\left(Z\left(f: f \in I(X)_{D}\right)\right) \leq N-i\right\},
$$

où $I(X)_{D}$ désigne la partie de degré $D$ de l'idéal homogène $I(X)$. Alternativement, $\omega_{i}(X)$ est le plus petit entier $D \geq 0$ tel qu'il existe des polynômes homogènes $f_{1}, \ldots, f_{i} \in I(X)$ de degré borné par $D$ formant une intersection complète. Évidemment $\omega(X)=\omega_{1}(X)$ et

$$
1 \leq \omega_{1}(X) \leq \cdots \leq \omega_{N-n}(X) .
$$

L'invariant $\omega(X)$ joue un rôle important dans les problèmes de Lehmer généralisé et de Bogomolov sur les tores [Dav03, AD04, Rat04. D'un autre côté, les lemmes de zéros 
consistent à minorer le premier indice $\omega(X)$ pour une variété de dimension 0 , éventuellement munie de multiplicités; ces résultats sont des outils fondamentaux en théorie des nombres transcendants, voir Ber87. Signalons que pour ces applications, il est souvent important de considérer des indices d'obstruction sur des sous-corps de $\overline{\mathbf{Q}}$ (i.e. dont les équations sont à coefficients dans un sous-corps fixé) et pour des schémas projectifs quelconques.

Plus généralement encore, on peut considérer des indices d'obstruction successifs relatifs à un ouvert donné $U \subset \mathbf{P}^{N}$ :

$$
\omega_{i}(X ; U):=\min \left\{D \in \mathbf{N}: \operatorname{dim}\left(Z\left(f: f \in I(X)_{D}\right) \cap U\right) \leq N-i\right\} .
$$

Les idéaux des variétés toriques sont binomiaux, c'est-à-dire engendrés par des famille de polynômes de la forme $\alpha x^{a}-\beta x^{b}$ avec $a, b \in \mathbf{N}^{n}$ et $\alpha, \beta \in \mathbf{K}^{\times}$. Dans la suite on explicite la relation entre variétés toriques et idéaux binomiaux, qui est la clé de notre étude des indices d'obstruction successifs de ces variétés.

Soit $\mathcal{A}=\left(a_{0}, \ldots, a_{N}\right) \in\left(\mathbf{Z}^{n}\right)^{N+1}$ tel que $L_{\mathcal{A}}=\mathbf{Z}^{n}$ et $\alpha=\left(\alpha_{0}, \ldots, \alpha_{N}\right) \in\left(\mathbf{K}^{\times}\right)^{N+1}$. Considérons l'application linéaire

$$
\eta_{\mathcal{A}}: \mathbf{Z}^{N+1} \rightarrow \mathbf{Z} \times \mathbf{Z}^{n}, \quad \lambda \mapsto\left(\lambda_{0}+\cdots+\lambda_{N}, \lambda_{0} a_{0}+\cdots+\lambda_{N} a_{N}\right),
$$

et posons $\Gamma_{\mathcal{A}}:=\operatorname{ker}\left(\eta_{\mathcal{A}}\right)$ son noyau. C'est un sous-module saturé de $\mathbf{Z}^{N+1}$ (c'est-à-dire que le quotient $\mathbf{Z}^{N+1} / \Gamma_{\mathcal{A}}$ est sans torsion) de rang $N-n$. Notons $\Delta \subset \mathbf{R}^{N+1}$ l'hyperplan d'équation $\lambda_{0}+\cdots+\lambda_{N}=0$ et posons $\Delta^{\mathbf{Z}}:=\Delta \cap \mathbf{Z}^{N+1}$; évidemment on a $\Gamma_{\mathcal{A}} \subset \Delta^{\mathbf{Z}}$.

Pour $b \in \mathbf{R}^{N+1}$ on écrit de façon unique $b=b_{+}-b_{-}$avec $b_{+}, b_{-} \in \mathbf{R}_{+}^{N+1}$ à supports disjoints, c'est-à-dire $\left(b_{+}\right)_{i}=b_{i}$ si $b_{i}>0$ et 0 sinon, et $\left(b_{-}\right)_{i}=-b_{i}$ si $b_{i}<0$ et 0 sinon. Le résultat suivant est une reformulation de [Stu96, Cor. 4.3]:

Proposition II.1. $-I\left(X_{\mathcal{A}, \alpha}\right)=\left(x^{b_{+}}-\alpha^{b} x^{b_{-}}: b \in \Gamma_{\mathcal{A}}\right)$.

Démonstration. - Le cône de $X_{\mathcal{A}, \alpha}$ coïncide avec l'adhérence de Zariski de l'image de

$$
\mathbf{G}_{m}^{n} \times \mathbf{G}_{m} \rightarrow \mathbf{A}^{N+1}, \quad(s, t)=\left(s_{1}, \ldots, s_{n}, t\right) \mapsto\left(\alpha_{0} t s^{a_{0}}, \ldots, \alpha_{N} t s^{a_{N}}\right),
$$

donc $I\left(X_{\mathcal{A}, \alpha}\right)$ coïncide avec le noyau de l'homomorphisme

$$
\mathbf{K}\left[x_{0}, \ldots, x_{N}\right] \rightarrow \mathbf{K}\left[s_{1}^{ \pm 1}, \ldots, s_{n}^{ \pm 1}, t^{ \pm 1}\right], \quad x_{i} \mapsto \alpha_{i} t s^{a_{i}} ;
$$

le résultat devient une conséquence directe de [Stu96, Cor. 4.3].

Ainsi, l'idéal d'une variété torique projective est binomial, il en résulte automatiquement que c'est un idéal premier et homogène (puisque $X_{\mathcal{A}, \alpha}$ est une variété projective) ne contenant aucune des variables $x_{i}$, à cause de l'hypothèse $\alpha \in\left(\mathbf{P}^{N}\right)^{\circ}$.

Soit maintenant $\Gamma \subset \mathbf{Z}^{N+1}$ un sous-module quelconque et $\rho$ un caractère partiel, c'est-à-dire un homomorphisme $\rho: \Gamma \rightarrow \mathbf{K}^{\times}$. Ces données définissent un idéal binomial

$$
I(\Gamma, \rho):=\left(x^{b+}-\rho(b) x^{b_{-}}: b \in \Gamma\right) \subset \mathbf{K}\left[x_{0}, \ldots, x_{N}\right] .
$$

Proposition II.2. - ([ES96, Cor. 2.6]) - La correspondence

$$
(\Gamma, \rho) \mapsto I(\Gamma, \rho)
$$

est une bijection entre les sous-modules saturés $\Gamma \subset \mathbf{Z}^{N+1}$ munis d'un caractère partiel $\rho$, et les idéaux de $\mathbf{K}\left[x_{0}, \ldots, x_{N}\right]$ binomiaux, premiers et ne contenant aucune des variables $x_{i}$. De plus $\operatorname{rang}_{\mathbf{Z}}(\Gamma)=\operatorname{rang}(I(\Gamma, \rho))$. 
On vérifie sans peine que $I(\Gamma, \rho)$ est homogène si et seulement si $\Gamma \subset \Delta^{\mathbf{z}}$. Ainsi, la donnée d'un couple $(\mathcal{A}, \alpha)$ définit un sous-module saturé $\Gamma_{\mathcal{A}} \subset \Delta^{\mathbf{Z}}$ et un caractère partiel $\rho_{\mathcal{A}, \alpha}: b \mapsto \alpha^{b}$; la proposition $\llbracket .1$ peut être reformulée sous la forme

$$
I\left(X_{\mathcal{A}, \alpha}\right)=I\left(\Gamma_{\mathcal{A}}, \rho_{\mathcal{A}, \alpha}\right) .
$$

Réciproquement, à partir d'un idéal binomial de $\mathbf{K}\left[x_{0}, \ldots, x_{N}\right]$ premier, homogène et ne contenant aucune des variables $x_{i}$, on peut construire $(\mathcal{A}, \alpha)$ tel que $I=I\left(X_{\mathcal{A}, \alpha}\right)$ : soient $\Gamma \subset$ $\Delta^{\mathbf{Z}}$ le sous-module saturé et $\rho$ le caractère partiel associés à $I, n:=N-\operatorname{rang}_{\mathbf{Z}}(\Gamma)$ et prenons $v_{0}, \ldots, v_{n} \in \mathbf{Z}^{N+1}$ une base de l'orthogonal $\Gamma^{\perp}$ de $\Gamma$ dans $\mathbf{Z}^{N+1}$ relativement au produit scalaire usuel. Puisque $\Gamma \subset \Delta^{\mathbf{Z}}$ on peut supposer sans perte de généralité $v_{0}=(1, \ldots, 1)$; on pose alors

$$
a_{i}:=\left(v_{1, i}, \ldots, v_{n, i}\right) \in \mathbf{Z}^{n} \quad \text { pour } i=0, \ldots, N,
$$

et $\mathcal{A}:=\left(a_{0}, \ldots, a_{N}\right) \in\left(\mathbf{Z}^{n}\right)^{N+1}$. En outre, $\rho$ peut s'étendre (de manière pas forcément unique) en un caractère total $\rho: \mathbf{Z}^{N+1} \rightarrow \mathbf{K}^{\times}$et on prend $\alpha:=\left(\rho\left(e_{0}\right), \ldots, \rho\left(e_{N}\right)\right) \in\left(\mathbf{K}^{\times}\right)^{N+1}$ où les $e_{i}$ désignent les vecteurs de la base standard de $\mathbf{Z}^{N+1}$.

Par construction $\Gamma_{\mathcal{A}}=\Gamma$ et $L_{\mathcal{A}}=\mathbf{Z}^{n}$, parce que $v_{0}, \ldots, v_{n}$ est une base de $\Gamma^{\perp}$ et $\Gamma$ est saturé. La proposition $\llbracket$ entraîne $I\left(X_{\mathcal{A}, \alpha}\right)=I$. En particulier, on en déduit que la correspondance $X \mapsto I(X)$ est une bijection entre l'ensemble des variétés toriques de $\mathbf{P}^{N}$ et l'ensemble des idéaux de $\mathbf{K}\left[x_{0}, \ldots, x_{N}\right]$ binomiaux, premiers, homogènes, ne contenant aucune des variables de $x_{i}, i=0, \ldots, N$. Dans la suite, on notera $\Gamma_{X}$ et $\rho_{X}$ le $\mathbf{Z}$-module et le caractère partiel associés à une variété torique donnée $X$.

Exemple II.3. - Soit

$$
\mathbf{G}_{m}^{2} \rightarrow \mathbf{P}^{3}, \quad(s, t) \mapsto\left(1: s: 3 s^{2} t: s t^{2}\right)
$$

et posons $S \subset \mathbf{P}^{3}$ la surface torique, adhérence de Zariski de l'image de cette application. Soit

$$
\left[\eta_{\mathcal{A}}\right]=\left[\begin{array}{llll}
1 & 1 & 1 & 1 \\
0 & 1 & 2 & 1 \\
0 & 0 & 1 & 2
\end{array}\right]
$$

la matrice de l'application linéaire $\eta_{\mathcal{A}}: \mathbf{Z}^{4} \rightarrow \mathbf{Z}^{3}$ dans les bases canoniques, alors $\Gamma_{\mathcal{A}}=\operatorname{ker}\left(\eta_{\mathcal{A}}\right)$ est engendré par le seul vecteur

$$
\gamma=\left(M_{0},-M_{1}, M_{2},-M_{3}\right)=(2,-3,2,-1) \in \mathbf{Z}^{4}
$$

où $M_{i}$ désigne le $i$-ème mineur de la matrice $\left[\eta_{\mathcal{A}}\right]$. Et donc une équation de $S$ est

$$
x^{\gamma_{+}}-\alpha^{\gamma} x^{\gamma_{-}}=x_{0}^{2} x_{2}^{2}-9 x_{1}^{3} x_{3} \in \mathbf{K}\left[x_{0}, x_{1}, x_{2}, x_{3}\right] .
$$

Réciproquement, partons de l'équation binomiale $f:=x_{0}^{2} x_{2}^{2}-9 x_{1}^{3} x_{3} \in \mathbf{K}\left[x_{0}, x_{1}, x_{2}, x_{3}\right]$. Le $\mathbf{Z}$-module saturé $\Gamma \subset \Delta^{\mathbf{Z}}$ correspondant est engendré par $\gamma=(2,-3,2,-1)$ et le caractère partiel est $\rho: \Gamma \rightarrow \mathbf{K}^{\times}, m \cdot \gamma \mapsto 9^{m}$.

On vérifie que $(1,1,1,1),(0,1,2,1),(0,0,1,2) \in \mathbf{Z}^{4}$ forment bien une base de $\Gamma^{\perp}$ et que $\mathbf{Z}^{4} \rightarrow \mathbf{K}^{\times}, b \mapsto(1,1,3,1)^{b}=3^{b_{2}}$, est une extension possible de $\rho$, donc $Z(f)=X_{\mathcal{A}, \alpha}$ avec $\mathcal{A}=((0,0),(1,0),(2,1),(1,2)) \in\left(\mathbf{Z}^{2}\right)^{4}$ et $\alpha=(1,1,3,1) \in\left(\mathbf{K}^{\times}\right)^{4}$.

La figure suivante montre les exposants et le polytope associés à cette surface: 


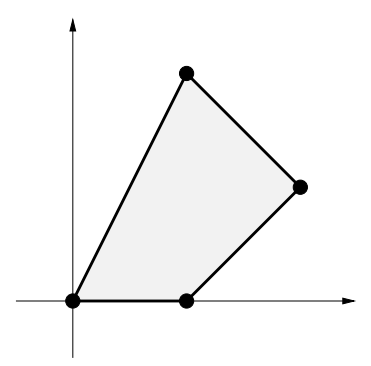

Considérons la norme $\ell^{1}$

$$
\|v\|_{1}=\sum_{i=0}^{N}\left|v_{i}\right| \quad \text { pour } v \in \mathbf{R}^{N+1}
$$

et pour un $\mathbf{Z}$-module quelconque $\Gamma \subset \mathbf{R}^{N+1}$ notons $\mu_{i}:=\mu_{i}\left(\Gamma ;\|\cdot\|_{1}\right)$ le $i$-ème minimum successif de $\Gamma$ relativement à cette norme. Rappelons que $\mu_{i}$ est défini comme le plus petit $\nu \in \mathbf{R}_{+}$tel qu'il existe $i$ vecteurs indépendants $v_{1}, \ldots, v_{i} \in \Gamma$ de norme bornée par $\nu$. Alternativement

$$
\mu_{i}\left(\Gamma ;\|\cdot\|_{1}\right):=\min \left\{\nu \in \mathbf{R}_{+}: \operatorname{rang}_{\mathbf{Z}}\left(\nu \cdot B_{\|\cdot\|_{1}} \cap \Gamma\right) \geq i\right\}
$$

où $B_{\|\cdot\|_{1}}$ désigne la boule unité pour la norme $\ell^{1}$. Le résultat suivant montre que les indices d'obstruction d'une variété torique relatifs à l'ouvert $\left(\mathbf{P}^{N}\right)^{\circ}$ coïncident avec les minimums successifs du Z-module associé:

Proposition II.4. - Soit $X \subset \mathbf{P}^{N}$ une variété torique de dimension n, alors

$$
\omega_{i}\left(X ;\left(\mathbf{P}^{N}\right)^{\circ}\right)=\frac{1}{2} \mu_{i}\left(\Gamma_{X} ;\|\cdot\|_{1}\right) \quad \text { pour } i=1, \ldots, N-n .
$$

Démonstration. - On peut se restreindre sans perte de généralité au cas $\alpha=(1, \ldots, 1)$. Soient $v_{1}, \ldots, v_{N-n}$ des vecteurs formant une base de $\Gamma_{X}$. D'après [ES96. Thm. 2.1(b)], $X^{\circ}=X \cap\left(\mathbf{P}^{N}\right)^{\circ}$ est une intersection complète decoupé par les binômes

$$
x^{\left(v_{1}\right)_{+}}-x^{\left(v_{1}\right)_{-}}, \ldots, x^{\left(v_{N-n}\right)_{+}}-x^{\left(v_{N-n}\right)_{-}} \in \mathbf{K}\left[x_{0}, \ldots, x_{N}\right]
$$

et on a $\operatorname{deg}\left(x^{\left(v_{j}\right)_{+}}-x^{\left(v_{j}\right)_{-}}\right)=\frac{1}{2}\left\|v_{j}\right\|_{1}\left(\operatorname{car} \sum_{k=0}^{N} v_{j, k}=0\right)$. En prenant $v_{1}, \ldots, v_{N-n}$ réalisant les minimums successifs du module $\Gamma_{X}$ on obtient

$$
\omega_{i}\left(X ;\left(\mathbf{P}^{N}\right)^{\circ}\right) \leq \max \left\{\operatorname{deg}\left(x^{\left(v_{j}\right)_{+}}-x^{\left(v_{j}\right)_{-}}\right): 1 \leq j \leq i\right\}=\frac{1}{2} \mu_{i}\left(\Gamma_{X} ;\|\cdot\|_{1}\right) .
$$

Dans l'autre direction, soit $f_{1}, \ldots, f_{N-n} \in I(X)$ une suite de polynômes homogènes réalisant les indices d'obstruction successifs de $X \operatorname{sur}\left(\mathbf{P}^{N}\right)^{\circ}$. Par la proposition \I.1

$$
f_{j}(x)=\sum_{b \in \Gamma_{X}} g_{j, b}(x)\left(x^{b_{+}}-x^{b_{-}}\right)
$$

pour certains $g_{j, b} \in \mathbf{K}\left[x_{0}, \ldots, x_{N}\right]$ et, pour tout $b \in \Gamma_{\mathcal{A}}$ tel que $g_{j, b} \neq 0$, on a

$$
\operatorname{deg}\left(f_{j}\right)=\operatorname{deg}\left(g_{j, b}\right)+\operatorname{deg}\left(x^{b_{+}}-x^{b_{-}}\right)=\operatorname{deg}\left(g_{j, b}\right)+\frac{1}{2}\|b\|_{1} \geq \frac{1}{2}\|b\|_{1} .
$$

Soit $1 \leq i \leq N-n$, posons $L_{i}$ le $\mathbf{Z}$-module engendré par les $b \in \Gamma_{X}$ tels que $g_{j, b} \neq 0$ pour un certain $1 \leq j \leq i$. Alors, $f_{j} \in J_{i}:=\left(x^{b_{+}}-x^{b_{-}}: b \in L_{i}\right) \subset \mathbf{K}\left[x_{0}, \ldots, x_{N}\right]$ pour $j=1, \ldots, i$ et donc

$$
i=\operatorname{rang}\left(f_{1}, \ldots, f_{i}\right) \leq \operatorname{rang}\left(J_{i}\right)=\operatorname{rang}_{\mathbf{Z}}\left(L_{i}\right)
$$


par la proposition $\llbracket .2$ ci-dessus. Ainsi $L_{i}$ est un sous-module de $\Gamma_{X}$ de rang au moins $i$ et on peut trouver $i$ vecteurs linéairement indépendants parmi les $b \in \Gamma_{\mathcal{A}}$ tels que $g_{j, b} \neq 0$ pour un certain $1 \leq j \leq i$. L'un au moins de ces vecteurs est de norme $\|b\|_{1} \geq \mu_{i}\left(\Gamma_{X} ;\|\cdot\|_{1}\right)$ et on en déduit avec (II.5)

$$
\omega_{i}\left(X ;\left(\mathbf{P}^{N}\right)^{\circ}\right)=\max \left\{\operatorname{deg}\left(f_{j}\right): j=1, \ldots, i\right\} \geq \frac{1}{2} \mu_{i}\left(\Gamma_{X},\|\cdot\|_{1}\right) .
$$

Soit $X \subset \mathbf{P}^{N}$ une variété de dimension $n$, comme conséquence directe de sa majoration de la fonction de Hilbert géométrique, Chardin a montré qu'on a toujours

$$
\omega_{1}(X) \leq N \operatorname{deg}(X)^{\frac{1}{N-n}}
$$

et aussi $\omega_{2}(X)^{\frac{1}{2}} \leq N \operatorname{deg}(X)^{\frac{1}{N-n}}$ Cha89. Ultérieurement, Chardin et Philippon ont considéré le problème de l'interpolation algébrique, qui consiste à estimer le degré minimal de sous-variétés

$$
X=Y_{N-n} \subset \cdots \subset Y_{1} \subset \mathbf{P}^{N}
$$

telles que $\operatorname{codim}\left(Y_{j}\right)=j$. Ils ont montré qu'on peut choisir $Y_{1}, \ldots, Y_{N-n}$ comme ci-dessus, satisfaisant CP99

$$
\operatorname{deg}\left(Y_{j}\right)^{\frac{1}{j}} \leq N 4^{N-1} \operatorname{deg}(X)^{\frac{1}{N-n}} .
$$

L'interpolation algébrique est très proche des problèmes d'estimation des indices d'obstruction successifs. En fait, une reformulation de leur démonstration montre qu'il existe des équations $f_{1}, \ldots, f_{N-n} \in I(X)$ formant une intersection complète au voisinage du point générique de $X$ et telles que

$$
\operatorname{deg}(X) \leq \operatorname{deg}\left(f_{1}\right) \cdots \operatorname{deg}\left(f_{N-n}\right) \leq c(N) \operatorname{deg}(X)
$$

pour une constante $c(N)>0$ explicite. Ceci équivaut aux inégalités

$$
\operatorname{deg}(X) \leq \omega_{1}(X ; U) \cdots \omega_{N-n}(X ; U) \leq c(N) \operatorname{deg}(X)
$$

pour un ouvert $U \subset \mathbf{P}^{N}$ tel que $X \cap U \neq \emptyset$. L'interprétation des indices d'obstruction des variétés toriques comme minimums successifs d'un module permet de traduire le deuxième théorème de Minkowski en des estimations pour le produit de ces indices relatifs à l'ouvert $\left(\mathbf{P}^{N}\right)^{\circ}$ :

Corollaire II.5. - Soit $X \subset \mathbf{P}^{N}$ une variété torique de dimension n, alors

$$
\operatorname{deg}(X) \leq \omega_{1}\left(X ;\left(\mathbf{P}^{N}\right)^{\circ}\right) \cdots \omega_{N-n}\left(X ;\left(\mathbf{P}^{N}\right)^{\circ}\right) \leq c(N, n) \operatorname{deg}(X)
$$

avec

$$
c(N, n):=\left(\begin{array}{c}
N+1 \\
n+1
\end{array}\right)^{\frac{1}{2}}\left(\frac{N+1}{\pi}\right)^{\frac{N-n}{2}} \Gamma\left(1+\frac{N-n}{2}\right) \leq\left(\frac{N+1}{\sqrt{\pi}}\right)^{N-n} .
$$

Ceci est la proposition 0.1 dans l'introduction, avec une constante plus précise.

Démonstration. - La première inégalité est conséquence directe du théorème de Bézout; passons à la deuxième. Posons $\Gamma_{X}^{\mathbf{R}}:=\Gamma_{X} \otimes \mathbf{R} \cong \mathbf{R}^{N-n}$, de sorte que $\Gamma_{X}$ est un réseau de $\Gamma_{X}^{\mathbf{R}}$. Posons aussi $B:=B_{\|\cdot\|_{1}} \cap \Gamma_{X}^{\mathbf{R}}$ la boule unité de $\Gamma_{X}^{\mathbf{R}}$ par rapport à la restriction de la norme $\ell^{1}$. Ceci étant un ensemble convexe et symétrique par rapport à l'origine, le deuxième théorème de Minkowski sur les minimums successifs d'un réseau entraîne

$$
\frac{2^{N-n}}{(N-n) !} \leq \frac{\operatorname{Vol}_{N-n}(B)}{\operatorname{Vol}_{N-n}\left(\Gamma_{X}^{\mathbf{R}} / \Gamma_{X}\right)} \prod_{i=1}^{N-n} \mu_{i}\left(\Gamma_{X} ;\|\cdot\|_{1}\right) \leq 2^{N-n}
$$


et donc

$$
\prod_{i=1}^{N-n} \omega_{i}\left(X ;\left(\mathbf{P}^{N}\right)^{\circ}\right) \leq \frac{\operatorname{Vol}_{N-n}\left(\Gamma_{X}^{\mathbf{R}} / \Gamma_{X}\right)}{\operatorname{Vol}_{N-n}(B)}
$$

par la proposition $\llbracket .4$

Pour conclure, il suffit de majorer le quotient $\operatorname{Vol}_{N-n}\left(\Gamma_{X}^{\mathbf{R}} / \Gamma_{X}\right) / \operatorname{Vol}_{N-n}(B)$. Soient $\mathcal{A} \in$ $\left(\mathbf{Z}^{n}\right)^{N+1}$ et $\alpha \in\left(\mathbf{K}^{\times}\right)^{N+1}$ tels que $X=X_{\mathcal{A}, \alpha}$; en particulier $\Gamma_{\mathcal{A}}=\Gamma_{X}$. Soient

$$
v_{i}:=\left(a_{0, i}, \ldots, a_{N, i}\right) \in \mathbf{Z}^{N+1} \quad \text { pour } i=0, \ldots, n
$$

les lignes de la matrice (dans les bases standard) de l'application $\eta_{\mathcal{A}}: \mathbf{Z}^{N+1} \rightarrow \mathbf{Z}^{n+1}$. Le module $\Gamma_{X}$ est l'orthogonal du sous-module $V \subset \mathbf{Z}^{N+1}$ engendré par $v_{0}, \ldots, v_{n}$. C'est aussi un sous-module saturé à cause de l'hypothèse $L_{\mathcal{A}}=\mathbf{Z}^{n}$; la formule de Brill-Gordan (voir par exemple [PS04, formule (II.2)]) entraîne alors

$$
\operatorname{Vol}_{N-n}\left(\Gamma_{X}^{\mathbf{R}} / \Gamma_{X}\right)=\operatorname{Vol}_{n+1}\left(V^{\mathbf{R}} / V\right)
$$

et par la formule de Cauchy-Binet

$$
\operatorname{Vol}_{N-n}\left(\Gamma_{X}^{\mathbf{R}} / \Gamma_{X}\right)=\operatorname{Vol}_{n+1}\left(V^{\mathbf{R}} / V\right)=\left\|v_{0} \wedge \cdots \wedge v_{n}\right\|_{2}=\left(\sum_{J: \operatorname{Card}(J)=n+1} \operatorname{det}\left(\left[\eta_{\mathcal{A}}\right]_{J}\right)^{2}\right)^{1 / 2}
$$

où $\left[\eta_{\mathcal{A}}\right]_{J}$ désigne le mineur $(n+1) \times(n+1)$ de la matrice de $\eta_{\mathcal{A}}$ dont les colonnes sont indexées par $J$. Chaque terme $\operatorname{det}\left(\left[\eta_{\mathcal{A}}\right]_{J}\right)$ est $\pm n$ ! fois le volume d'un simplexe contenu dans le polytope $Q_{\mathcal{A}}$; ainsi on peut majorer cette quantité par

$$
\operatorname{Vol}_{N-n}\left(\Gamma_{X}^{\mathbf{R}} / \Gamma_{X}\right) \leq\left(\begin{array}{c}
N+1 \\
n+1
\end{array}\right)^{1 / 2} n ! \operatorname{Vol}_{n}\left(Q_{\mathcal{A}}\right)=\left(\begin{array}{c}
N+1 \\
n+1
\end{array}\right)^{1 / 2} \operatorname{deg}(X)
$$

En outre, notons que $B$ contient la boule euclidienne de $\Gamma_{X}^{\mathbf{R}}$ de rayon $(N+1)^{-1 / 2}$ centrée en l'origine, ainsi

$$
\operatorname{Vol}_{N-n}(B) \geq \operatorname{Vol}_{N-n}\left((N+1)^{-1 / 2} B_{\|\cdot\|_{2}} \cap \Gamma_{X}^{\mathbf{R}}\right)=\left(\frac{\pi}{N+1}\right)^{\frac{N-n}{2}} \cdot \frac{1}{\Gamma\left(1+\frac{N-n}{2}\right)} .
$$

On en déduit

$$
\begin{aligned}
\prod_{i=1}^{N-n} \omega_{i}\left(X ;\left(\mathbf{P}^{N}\right)^{\circ}\right) & \leq \frac{\operatorname{Vol}_{N-n}\left(\Gamma_{X}^{\mathrm{R}} / \Gamma_{X}\right)}{\operatorname{Vol}_{N-n}(B)} \\
& \leq\left(\begin{array}{c}
N+1 \\
n+1
\end{array}\right)^{\frac{1}{2}} \cdot\left(\frac{N+1}{\pi}\right)^{\frac{N-n}{2}} \cdot \Gamma\left(1+\frac{N-n}{2}\right) \cdot \operatorname{deg}(X) \\
& \leq \frac{(N+1)^{\frac{N-n}{2}}}{(N-n)^{\frac{1}{2}}} \cdot\left(\frac{N+1}{\pi}\right)^{\frac{N-n}{2}} \cdot\left[\prod_{k=0}^{\left[\frac{N-n}{2}\right]-1}\left(\frac{N-n}{2}-k\right) \cdot \operatorname{deg}(X)\right. \\
& \leq\left(\frac{N+1}{\sqrt{\pi}}\right)^{N-n} \cdot \operatorname{deg}(X) .
\end{aligned}
$$

Ce résultat améliore la constante $c(N)$ dans (II.7) pour le cas torique et précise l'ouvert $U$ par rapport auquel les indices peuvent être considérés.

En revenant au cas général d'une variété $X \subset \mathbf{P}^{N}$ quelconque de dimension $n$, il est naturel de se demander si l'estimation (III.7) reste valide pour $U=\mathbf{P}^{N}$. Autrement-dit, s'il existe 
toujours des polynômes homogènes $f_{1}, \ldots, f_{N-n} \in I(X)$ formant une intersection complète globale et tels que

$$
\operatorname{deg}(X) \leq \operatorname{deg}\left(f_{1}\right) \cdots \operatorname{deg}\left(f_{N-n}\right) \leq c(N) \operatorname{deg}(X) .
$$

Il serait intéressant de décider cette question déjà sur la classe des variétés toriques. Pour ce faire, on voudrait expliciter les indices $\omega_{i}\left(X ; \mathbf{P}^{N}\right)$ de manière analogue à la proposition ஹ.4 pour $\omega_{i}\left(X ;\left(\mathbf{P}^{N}\right)^{\circ}\right)$.

\section{Volumes, hauteurs d'espaces tangents et degrés}

Dans la définition du $\S$ 【 les sous-groupes algébriques connexes de $\mathbf{G}_{m}^{N}$ correspondent aux variétés toriques $X_{\mathcal{A}}^{\circ}$ via l'identification $\iota: \mathbf{G}_{m}^{N} \hookrightarrow\left(\mathbf{P}^{N}\right)^{\circ}$. D'un autre côté, l'ensemble des points complexes d'un sous-groupe algébrique connexe $G$ de $\mathbf{G}_{m}^{N}$, de dimension $n$, est décrit via l'application exponentielle par son espace tangent $T G(\mathbf{C}) \subset \mathbf{C}^{N}$ à l'origine et son réseau de périodes

$$
\Lambda:=T G(\mathbf{C}) \cap(2 \mathrm{i} \pi \mathbf{Z})^{N},
$$

de rang $n$ sur $\mathbf{Z}$.

Munissons l'espace tangent à $\mathbf{G}_{m}^{N}$ en l'origine $T \mathbf{G}_{m}^{N}(\mathbf{C}) \simeq \mathbf{C}^{N}$ de la structure euclidienne pour laquelle les $2 N$ éléments $e_{i}$ et $2 \mathrm{i} \pi e_{i}(i=1, \ldots, N)$ forment une base orthonormée.

Soit $\mathcal{A}:=\left(a_{0}, \ldots, a_{N}\right) \in\left(\mathbf{Z}^{n}\right)^{N+1}$ tel que $L_{\mathcal{A}}=\mathbf{Z}^{n}$ et $G:=\iota^{-1}\left(X_{\mathcal{A}}^{\circ}\right)$. Des générateurs de $\Lambda$ sont donnés par les vecteurs $2 \mathrm{i} \pi \lambda_{1}, \ldots, 2 \mathrm{i} \pi \lambda_{n} \in(2 \mathrm{i} \pi \mathbf{Z})^{N}$ où $\lambda_{i, j}=a_{j, i}-a_{0, i}$ pour $i=1, \ldots, n$ et $j=1, \ldots, N$. En fait, le $\mathbf{Z}$-module $\frac{1}{2 \mathrm{i} \pi} \Lambda$ est l'orthogonal de la projection par $\pi: \mathbf{R}^{N+1} \rightarrow \mathbf{R}^{N},\left(x_{0}, x_{1}, \ldots, x_{N}\right) \mapsto\left(x_{1}, \ldots, x_{N}\right)$, du Z Z-module $\Gamma_{\mathcal{A}} \subset \Delta^{\mathbf{Z}}$, introduit au $\S$ II précédent.

Considérons encore l'adhérence de Zariski $\bar{G}$ de $G$ dans la compactification $\left(\mathbf{P}^{1}\right)^{N}$ de $\mathbf{G}_{m}^{N}$, ses multidegrés sont indexés par des multi-indices $c=\left(c_{1}, \ldots, c_{N}\right) \in \mathbf{N}_{n}^{N}$ où $c_{j} \in\{0,1\}$. À toute suite croissante $1 \leq j_{1}<\cdots<j_{n} \leq N$ on associe un multi-indice $c\left(j_{1}, \ldots, j_{n}\right)$ défini par $c\left(j_{1}, \ldots, j_{n}\right)_{j}=1$ si $j \in\left\{j_{1}, \ldots, j_{n}\right\}$ et 0 sinon. Indiquons comment on retrouve par ce biais la proposition 4 de [BP88.

Proposition III.1. - Avec les notation ci-dessus, le volume euclidien de la projection de $\Lambda$ sur le produit des $n$ facteurs de $\mathbf{R}^{N}$ indexés par $j_{1}, \ldots, j_{n}$ est égal à $\mathrm{MV}_{n}\left(\overline{a_{0} a_{j_{1}}}, \ldots, \overline{a_{0} a_{j_{n}}}\right)=$ $\operatorname{deg}_{c\left(j_{1}, \ldots, j_{n}\right)}(\bar{G})$, où $\overline{a_{0} a_{j}}$ désigne le segment de droite joignant $a_{0} \grave{a} a_{j}$ dans $\mathbf{R}^{n}$.

Démonstration. - En effet, la projection de $\Lambda$ considérée est un réseau de $(2 \mathrm{i} \pi \mathbf{R})^{n}$ engendré par les vecteurs $\left(2 \mathrm{i} \pi \lambda_{i, j_{1}}, \ldots, 2 \mathrm{i} \pi \lambda_{i, j_{n}}\right)$ pour $i=1, \ldots, n$. Son volume pour la structure euclidienne fixée est donc égal à $\left|\Lambda_{j_{1}, \ldots, j_{n}}\right|=\operatorname{MV}_{n}\left(\overline{a_{0} a_{j_{1}}}, \ldots, \overline{a_{0} a_{j_{n}}}\right)$ où

$$
\Lambda_{j_{1}, \ldots, j_{n}}=\operatorname{det}\left[\begin{array}{ccc}
\lambda_{1, j_{1}} & \ldots & \lambda_{1, j_{n}} \\
\vdots & & \vdots \\
\lambda_{n, j_{1}} & \ldots & \lambda_{n, j_{n}}
\end{array}\right] \in \mathbf{Z} \text {. }
$$

Finalement, par la formule (1.2) ce multivolume est égal au multidegré $\operatorname{deg}_{c\left(j_{1}, \ldots, j_{n}\right)}(\bar{G})$ correspondant.

On notera que les $\Lambda_{j_{1}, \ldots, j_{n}}$ introduits dans la démonstration précédente sont des coordonnées grasmaniennes de l'espace tangent $T G(\mathbf{C})$ en l'origine de $G$ dans $\mathbf{C}^{N}$. Comme $\Lambda$ est un réseau primitif de $T G(\mathbf{C})$ on a

$$
\operatorname{ppcm}\left(\Lambda_{j_{1}, \ldots, j_{n}}: 1 \leq j_{1}<\cdots<j_{n} \leq N\right)=1 .
$$


L'image de $G$ par le plongement de Segre $s:\left(\mathbf{P}^{1}\right)^{N} \rightarrow \mathbf{P}^{2^{N}-1}$ est décrite par la somme de Minkowski

$$
\overline{a_{0} a_{1}}+\cdots+\overline{a_{0} a_{N}} \subset \mathbf{R}^{n}
$$

dont le volume est égal à la somme des volumes mixtes pour toutes les projections du type envisagé dans la proposition 11.1 En particulier

$$
\begin{aligned}
\operatorname{deg}_{\mathbf{P}_{2^{N}-1}}(\overline{s(G)}) & =n ! \operatorname{Vol}_{n}\left(\overline{a_{0} a_{1}}+\cdots+\overline{a_{0} a_{N}}\right) \\
& =n ! \sum_{1 \leq j_{1}<\cdots<j_{n} \leq N} \operatorname{MV}_{n}\left(\overline{a_{0} a_{j_{1}}}, \ldots, \overline{a_{0} a_{j_{n}}}\right) \\
& =n ! \sum_{1 \leq j_{1}<\cdots<j_{n} \leq N}\left|\Lambda_{j_{1}, \ldots, j_{n}}\right| .
\end{aligned}
$$

Ainsi, le degré de $\overline{s(G)}$ est égal à $n$ ! fois la hauteur associée à la norme $\ell^{1}$ des coordonnées grasmaniennes de son espace tangent dans $\mathbf{C}^{N}$.

Si l'on considère la hauteur de Schmidt $h_{S}(T)$ d'un sous-espace $T \subset \overline{\mathbf{Q}}^{N}$ définie comme la hauteur projective du point qui le représente dans la variété grasmanienne [Sch91, page 28], on a la formule

$$
h_{S}(T G)=\left(\sum_{1 \leq j_{1}<\cdots<j_{n} \leq N}\left|\Lambda_{j_{1}, \ldots, j_{n}}\right|^{2}\right)^{\frac{1}{2}}=\operatorname{Vol}_{n}\left(T G(\mathbf{C}) \cap(2 \mathrm{i} \pi \mathbf{R})^{N} / \Lambda\right)=\operatorname{Vol}_{N-n}\left(\Gamma_{\mathcal{A}}^{\mathbf{R}} / \Gamma_{\mathcal{A}}\right)
$$

car $T G(\mathbf{C}) \subset \mathbf{C}^{N}$ est défini sur $\mathbf{Q}$ et ses coordonnées grasmaniennes $\Lambda_{j_{1}, \ldots, j_{n}}$ dans $\mathbf{Z}$ sont premières entre elles dans leur ensemble. La seconde égalité n'est autre que la formule de Cauchy-Binet déjà utilisée au $\S$ II La troisième égalité s'obtient en identifiant l'hyperplan $\Delta=\left\{\lambda_{0}+\cdots+\lambda_{N}=0\right\} \subset \mathbf{R}^{N+1}$ avec $\mathbf{R}^{N}$ par la projection sur les $N$ dernières coordonnées, de sorte que l'image de $\Delta^{\mathbf{Z}}$ est $\mathbf{Z}^{N}$.

En remarquant que $Q_{\mathcal{A}}=\operatorname{Conv}\left(a_{0}, \ldots, a_{N}\right)$ contient tous les simplexes de sommets pris parmi les $a_{i}$ et est contenu dans l'union de ces mêmes simplexes ayant $a_{0}$ comme sommet fixe, on vérifie facilement (le volume d'un simplexe $\operatorname{Conv}\left(a_{0}, a_{i_{1}}, \ldots, a_{i_{n}}\right)$ est égal à $\left.\frac{1}{n !}\left|\Lambda_{i_{1}, \ldots, i_{n}}\right|\right)$

$$
\frac{1}{n !} \max _{1 \leq j_{1}, \ldots, j_{n} \leq N}\left|\Lambda_{j_{1}, \ldots, j_{n}}\right| \leq \operatorname{Vol}_{n}\left(Q_{\mathcal{A}}\right) \leq \frac{1}{n !} \sum_{1 \leq j_{1}<\cdots<j_{n} \leq N}\left|\Lambda_{j_{1}, \ldots, j_{n}}\right| .
$$

D'après la formule (1.1), on sait que $\operatorname{deg}_{\mathbf{P}^{N}}(\bar{G})=n ! \operatorname{Vol}_{n}\left(Q_{\mathcal{A}}\right)$ et en particulier

Proposition III.2. - Pour tout sous-groupe algébrique $G \subset \mathbf{G}_{m}^{N}$ de dimension $n$ on a

$$
n ! \operatorname{deg}_{\mathbf{P}^{N}}(\bar{G}) \leq \operatorname{deg}_{\mathbf{P}^{2^{N}-1}}(\overline{s(G)}) \leq n !\left(\begin{array}{c}
N \\
n
\end{array}\right) \operatorname{deg}_{\mathbf{P}^{N}}(\bar{G}) .
$$

On pourra comparer avec le résultat analogue pour la hauteur normalisée dans DP99, Prop. 2.2].

Si l'on introduit la fonction $f_{\mathcal{A}}$ sur l'enveloppe convexe $Q_{\mathcal{A}}$ des points $\mathcal{A}=\left(a_{0}, \ldots, a_{N}\right)$ dans $\mathbf{R}^{n}$, à valeur dans $\mathbf{N}$, définie pour $u \in Q_{\mathcal{A}}$ par :

$$
f_{\mathcal{A}}(u):=\operatorname{Card}\left\{\left(j_{1}, \ldots, j_{n}\right) ; 1 \leq j_{1}<\cdots<j_{n} \leq N \text { et } u \in \operatorname{Conv}\left(a_{0}, a_{j_{1}}, \ldots, a_{j_{n}}\right)\right\},
$$

on vérifie facilement l'égalité

$$
\frac{\operatorname{deg}_{\mathbf{P}^{2}{ }^{N}}(\overline{s(G)})}{n ! \operatorname{deg}_{\mathbf{P}^{N}}(\bar{G})}=\frac{1}{\operatorname{Vol}_{n}\left(Q_{\mathcal{A}}\right)} \cdot \int_{Q_{\mathcal{A}}} f_{\mathcal{A}}(u) d u .
$$


Exemple III.3. - En considérant des points $a_{0}, \ldots, a_{N}$ dans $\mathbf{Z}^{n}$ tels que $a_{n+1}, \ldots, a_{N}$ soient très proches de $a_{0}$ comparativement à $a_{1}, \ldots, a_{n}$ (voir figure ci-dessous pour $n=2$ ), on a

$$
\sum_{1 \leq j_{1}<\cdots<j_{n} \leq N}\left|\Lambda_{j_{1}, \ldots, j_{n}}\right| \approx \max _{1 \leq j_{1}, \ldots, j_{n} \leq N}\left|\Lambda_{j_{1}, \ldots, j_{n}}\right|=\left|\Lambda_{1, \ldots, n}\right|
$$

On obtient ainsi une variété torique pour laquelle $n ! \operatorname{deg}_{\mathbf{P}_{N}}(\bar{G}) \approx \operatorname{deg}_{\mathbf{P}_{2^{N}-1}}(\overline{s(G)})$, montrant que l'inégalité de gauche dans la proposition III.2 est optimale.

Exemple III.4. - Considérons maintenant $N=m n$ points $a_{1}, \ldots, a_{N}$ de $\mathbf{Z}^{n}$ également concentrés autour de $a_{1}, \ldots, a_{n}$, par exemple $a_{k n+i} \approx a_{i}$ à l'intérieur de $\operatorname{Conv}\left(a_{0}, a_{1}, \ldots, a_{n}\right)$, pour $k=1, \ldots, m-1$ et $i=1, \ldots, n$ (voir figure ci-dessous pour $n=2$ ). On a alors

$$
\sum_{1 \leq j_{1}<\cdots<j_{n} \leq N}\left|\Lambda_{j_{1}, \ldots, j_{n}}\right| \gtreqless\left(\frac{N}{n}\right)^{n} n ! \operatorname{Vol}_{n}\left(a_{0}, a_{1}, \ldots, a_{n}\right) .
$$

On obtient ainsi une variété torique pour laquelle $\operatorname{deg}_{\mathbf{P}_{2^{N}-1}}(\overline{s(G)}) \gtrsim n !\left(\frac{N}{n}\right)^{n} \operatorname{deg}_{\mathbf{P}_{N}}(\bar{G})$, laissant supposer que l'inégalité de droite dans la proposition III.Q pourrait être améliorée d'un facteur $\mathrm{e}^{n}$. De fait, lorsque $n=2$ on vérifie pour toute configuration $\mathcal{A}$ de points et tout $u \in Q_{\mathcal{A}}$ la majoration $f_{\mathcal{A}}(u) \leq\left(\frac{N}{2}\right)^{2}$, d'où $\operatorname{deg}_{\mathbf{P}_{2^{N-1}}}(\overline{s(G)}) \leq 2\left(\frac{N}{2}\right)^{2} \operatorname{deg}_{\mathbf{P}_{N}}(\bar{G})$, qui est optimal.

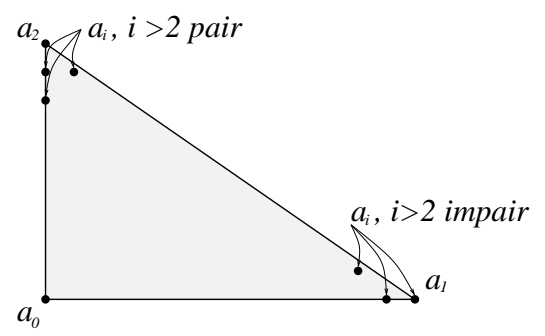

\section{Un théorème de Bézout pour les poids de Chow}

Soit $X \subset \mathbf{P}^{N}$ et $\tau=\left(\tau_{0}, \ldots, \tau_{N}\right) \in \mathbf{R}^{N+1}$, rappelons que $e_{\tau}(X) \in \mathbf{R}$ désigne le $\tau$-poids de Chow de $X$ défini dans l'introduction.

Pour $\lambda \in \mathbf{R}^{+}$on a $e_{\lambda \tau}(X)=\lambda e_{\tau}(X)$. De même, on vérifie facilement que pour $\tau^{\prime} \in$ $\mathbf{R}$ on a $e_{\tau+\left(\tau^{\prime}, \ldots, \tau^{\prime}\right)}(X)=e_{\tau}(X)+\tau^{\prime}(n+1) \operatorname{deg}(X)$. On vérifie encore que si $\sigma_{\delta}: \mathbf{P}^{N} \rightarrow$ $\mathbf{P}^{M}, M+1=\left(\begin{array}{c}N+\delta \\ N\end{array}\right)$, désigne le plongement de Veronese de degré $\delta$ et $\tau^{(\delta)}$ le vecteur des $\tau$-poids des monômes de degrés $\delta$, on a $e_{\tau^{(\delta)}}\left(\sigma_{\delta}(X)\right)=\delta^{n+1} \cdot e_{\tau}(X)$. Plus profonde est l'expression de $e_{\tau}(X)$ comme coefficient dominant d'une fonction poids de Hilbert démontrée par Mumford Mum77, Prop. 2.11] :

$$
s_{\tau}(X ; D):=\max _{J} \sum_{\lambda \in J}\left(\tau_{0} \lambda_{0}+\cdots+\tau_{N} \lambda_{N}\right)=\frac{e_{\tau}(X)}{(n+1) !} D^{n+1}+O\left(D^{n}\right),
$$

où le maximum porte sur tous les ensembles $J$ d'éléments de $\mathbf{N}_{D}^{N+1}$ tels que les monômes associés induisent une base de la partie graduée de degré $D$ de l'anneau de la variété $X$. 
Exemple IV.1. - Si $H$ est une hypersurface de $\mathbf{P}^{N}$ d'équation $f \in \mathbf{K}\left[x_{0}, \ldots, x_{N}\right]$ et $\tau \in$ $\mathbf{R}^{N+1}$, on a

$$
e_{\tau}(H)=\left(\tau_{0}+\cdots+\tau_{N}\right) \operatorname{deg}(H)-w_{t}\left(\lambda_{\tau}^{*}(f)\right)
$$

où $\lambda_{\tau}^{*}(f):=f\left(t^{\tau_{0}} x_{0}, \ldots, t^{\tau_{N}} x_{N}\right)$ et $w_{t}$ désigne la valuation $t$-adique.

Lorsque $\tau \in \mathbf{Z}^{N+1}$ le poids de Chow peut s'interpréter en termes de bidegré de la déformation torique $X_{\tau} \subset \mathbf{P}^{1} \times \mathbf{P}^{N}$ de $X$ relative à $\tau$. Une forme résultante d'indice $(1, n+1)$ de $X_{\tau}$ s'écrit

$$
v_{1}^{e_{\tau}(X)} v_{0}^{e_{-\tau}(X)} C h_{X}\left(\ldots,\left(-v_{0} / v_{1}\right)^{\tau_{j}} u_{i, j}, \ldots\right),
$$

son degré en $\left(v_{0}, v_{1}\right)$ est $e_{\tau}(X)+e_{-\tau}(X)$ d'après la définition du poids de Chow et c'est par ailleurs le bidegré $\operatorname{deg}_{(0, n+1)}\left(X_{\tau}\right)$, d'où

$$
\operatorname{deg}_{(0, n+1)}\left(X_{\tau}\right)=e_{\tau}(X)+e_{-\tau}(X) .
$$

Rappelons que le degré $\operatorname{deg}_{(0, n+1)}$ est obtenu en intersectant par $n+1$ formes linéaires relevées du second facteur $\mathbf{P}^{2 N+1}$, voir $\S$ 凹 pour plus de détails.

Lorsque $\tau \in \mathbf{N}^{N+1}$ on peut aussi interpréter le poids de Chow $e_{\tau}(X)$ isolément comme un bidegré en considérant une variation de la déformation torique précédente. Précisément, il s'agit maintenant de l'adhérence de Zariski $\widetilde{X}_{\tau} \subset \mathbf{P}^{1} \times \mathbf{P}^{2 N+1}$ de

$$
\left\{(1: t) \times\left(t^{\tau_{0}} x_{0}: \cdots: t^{\tau_{N}} x_{N}: x_{0}: \cdots: x_{N}\right) ; t \in \mathbf{G}_{m}, x \in X\right\} .
$$

En appliquant ce qui précède on a

$$
\operatorname{deg}_{(0, n+1)}\left(\widetilde{X}_{\tau}\right)=e_{\widetilde{\tau}}(\widetilde{X})+e_{-\widetilde{\tau}}(\widetilde{X}),
$$

où $\widetilde{X}$ désigne le plongement diagonal de $X$ dans $\mathbf{P}^{2 N+1}$ et $\widetilde{\tau}=\left(\tau_{0}, \ldots, \tau_{N}, 0, \ldots, 0\right) \in \mathbf{N}^{2 N+2}$. On vérifie sans difficulté $e_{\widetilde{\tau}}(\widetilde{X})=e_{\tau}(X)$ et $e_{-\widetilde{\tau}}(\widetilde{X})=0$, d'où $\operatorname{deg}_{(0, n+1)}\left(\widetilde{X}_{\tau}\right)=e_{\tau}(X)$.

Donnons maintenant la démonstration du théorème [0.2, en commençant par une variante de ce théorème de Bézout pour les poids de Chow. Notons

$$
\pi: \mathbf{P}^{1} \times \mathbf{P}^{2 N+1} \rightarrow \mathbf{P}^{N}, \quad\left(\left(t_{0}: t_{1}\right),\left(x_{0}: \cdots: x_{N}: y_{0}: \cdots: y_{N}\right)\right) \mapsto\left(y_{0}: \cdots: y_{N}\right)
$$

la projection de $\mathbf{P}^{1} \times \mathbf{P}^{2 N+1}$ sur $\mathbf{P}^{N}$ donnée par les $N+1$ dernières coordonnées de $\mathbf{P}^{2 N+1}$ et

$$
\tilde{\iota}: \mathbf{P}^{N} \rightarrow \mathbf{P}^{1} \times \mathbf{P}^{2 N+1} \quad, \quad\left(x_{0}: \cdots: x_{N}\right) \mapsto\left((0: 1),\left(x_{0}: \cdots: x_{N}: 0: \cdots: 0\right)\right) .
$$

Théorème $\boldsymbol{I} \boldsymbol{V}$.2. - Soit $X \subset \mathbf{P}^{N}$ une variété projective et $H$ un diviseur de $\mathbf{P}^{N}$ ne contenant pas $X$, alors pour tout $\tau \in \mathbf{Z}^{N+1}$ on a

$$
e_{\tau}(X \cdot H)=e_{\tau}(X) \operatorname{deg}(H)-\sum_{Y \in \operatorname{Irr}\left(\operatorname{init}_{\tau}(X)\right)} m\left(\tilde{X}_{\tau} \cdot \pi^{*}(H) ; \tilde{\iota}(Y)\right) \operatorname{deg}(Y)
$$

De plus, si $H$ est une hypersurface et $f \in \mathbf{K}\left[x_{0}, \ldots, x_{N}\right]$ est une équation de $H$ on a, avec les notations du théorème 0.2.

$$
m\left(\tilde{X}_{\tau} \cdot \pi^{*}(H) ; \tilde{\iota}(Y)\right)=m\left(X_{\tau} \cdot \operatorname{div}\left(\lambda_{\tau}^{*}(f)\right) ; \iota(Y)\right)
$$

où $\lambda_{\tau}^{*}(f):=f\left(t_{0}^{\tau_{0}} x_{0}, \ldots, t_{0}^{\tau_{N}} x_{N}\right) \in \mathbf{K}\left[t_{0}, t_{1}\right]\left[x_{0}, \ldots, x_{N}\right]$.

Démonstration. - Il suffit d'établir le résultat pour $\tau \in \mathbf{N}^{N+1}$, on se ramène à ce cas en ajoutant à $\tau$ un vecteur $\left(\tau^{\prime}, \ldots, \tau^{\prime}\right)$ où $\tau^{\prime} \in \mathbf{N}$ est suffisamment grand et on remarque qu'avec les propriétés d'homogénéité du poids de Chow l'égalité désirée reste invariante.

Dans ce cas, comme $H$ ne contient pas $X$, le cycle intersection $\widetilde{X}_{\tau} \cdot \pi^{*}(H)$ s'écrit comme la somme du cycle $(\widetilde{X \cdot H})_{\tau}$ et d'un cycle supporté par $\tilde{\iota}\left(\operatorname{init}_{\tau}(X)\right)$, le second vivant donc dans 
$\{(0: 1)\} \times \mathbf{P}^{2 N+1}$. Comme $\operatorname{deg}_{(0, n+1)}\left(\widetilde{X}_{\tau}\right)=e_{\tau}(X)$ et $\left.\operatorname{deg}_{(0, n)}(\widetilde{(X \cdot H})_{\tau}\right)=e_{\tau}(X \cdot H)$ on a, d'après le théorème de Bézout multi-projectif [Rem01b, Thm. 3.4],

$$
\begin{aligned}
\operatorname{deg}(H) e_{\tau}(X) & =\operatorname{deg}(H) \operatorname{deg}_{(0, n+1)}\left(\widetilde{X}_{\tau}\right) \\
& =\operatorname{deg}_{(0, n)}\left(\widetilde{X}_{\tau} \cdot \pi^{*}(H)\right) \\
& =\operatorname{deg}_{(0, n)}\left((X \cdot H)_{\tau}\right)+\sum_{Y \in \operatorname{Irr}\left(\operatorname{init}_{\tau}(X)\right)} m\left(\widetilde{X}_{\tau} \cdot \pi^{*}(H) ; \tilde{\iota}(Y)\right) \cdot \operatorname{deg}(Y) \\
& =e_{\tau}(X \cdot H)+\sum_{Y \in \operatorname{Irr}\left(\operatorname{init}_{\tau}(X)\right)} m\left(\widetilde{X}_{\tau} \cdot \pi^{*}(H) ; \tilde{\iota}(Y)\right) \cdot \operatorname{deg}(Y) .
\end{aligned}
$$

Notons finalement que le morphisme

$$
\pi^{\prime}: \begin{array}{ccc}
\mathbf{P}^{1} \times \mathbf{P}^{2 N+1} & \rightarrow & \mathbf{P}^{1} \times \mathbf{P}^{N} \\
\left(\left(t_{0}: t_{1}\right),\left(x_{0}: \cdots: x_{N}: y_{0}: \cdots: y_{N}\right)\right) & \mapsto & \left(\left(t_{0}: t_{1}\right),\left(x_{0}: \cdots: x_{N}\right)\right)
\end{array}
$$

est un isomorphisme de $\widetilde{X}_{\tau}$ sur $X_{\tau}$ dans l'ouvert $t_{1} \neq 0$, tel que $\pi^{\prime} \circ \tilde{\iota}=\iota$ et $\pi_{*}^{\prime}\left(\pi^{*}(H)\right)=$

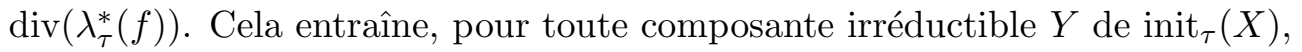

$$
m\left(\widetilde{X}_{\tau} \cdot \pi^{*}(H) ; \tilde{\iota}(Y)\right)=m\left(X_{\tau} \cdot \operatorname{div}\left(\lambda_{\tau}^{*}(f)\right) ; \iota(Y)\right)
$$

Démonstration du théorème 0.2 - On reprend la fin de la démonstration du théorème IV.2 en se placant dans une carte affine contenant une composante $Y \operatorname{de}^{\operatorname{init}_{\tau}}(X)$ et sur laquelle $H$ est décrit par une équation $f$, on a

$$
\begin{aligned}
m\left(\tilde{X}_{\tau} \cdot \pi^{*}(H) ; \tilde{\iota}(Y)\right) & =\operatorname{long}\left(\mathbf{K}\left[\tilde{X}_{\tau}\right] /\left(\pi^{*}(f)\right)\right)_{\tilde{\imath}(Y)} \\
& =\operatorname{long}\left(\mathbf{K}\left[X_{\tau}\right] /\left(\lambda_{\tau}^{*}(f)\right)\right)_{\iota(Y)} \\
& =m\left(X_{\tau} \cdot \operatorname{div}\left(\lambda_{\tau}^{*}(f)\right) ; \iota(Y)\right) \\
& =m\left(X_{\tau} \cdot H_{\tau} ; \iota(Y)\right)+w_{t_{0}}\left(\lambda_{\tau}^{*}(f)\right) m\left(\operatorname{init}_{\tau}(X) ; Y\right) \\
& =m\left(X_{\tau} \cdot H_{\tau} ; \iota(Y)\right)+\left(\left(\tau_{0}+\cdots+\tau_{N}\right) \operatorname{deg}(H)-e_{\tau}(H)\right) m\left(\operatorname{init}_{\tau}(X) ; Y\right)
\end{aligned}
$$

d'après le calcul de l'exemple IV.1 Enfin, en sommant sur toutes les composantes $Y$ de $\operatorname{init}_{\tau}(X)$ on a $\sum_{Y} m\left(\operatorname{init}_{\tau}(X) ; Y\right) \operatorname{deg}(Y)=\operatorname{deg}\left(\operatorname{init}_{\tau}(X)\right)=\operatorname{deg}(X)$.

Dans les notations du théorème [V.2 soit $f \in \mathbf{K}\left[x_{0}, \ldots, x_{N}\right]$ une équation de $H$ et $\tau \in$ $\mathbf{R}^{N+1}$, posons

$$
w_{X, \tau}(f):=\frac{1}{\operatorname{deg}(X)} \cdot\left(e_{\tau}(X \cdot H)-e_{\tau}(X) \operatorname{deg}(H)\right),
$$

c'est une fonction continue de $\tau$. Lorsque $\tau \in \mathbf{N}^{N+1}$ on a donc par le théorème IV.2

$$
w_{X, \tau}(f)=-\sum_{Y \in \operatorname{Irr}\left(\operatorname{init}_{\tau}(X)\right)} m\left(\tilde{X}_{\tau} \cdot \pi^{*}(H) ; \tilde{\iota}(Y)\right) \cdot \frac{\operatorname{deg}(Y)}{\operatorname{deg}(X)} .
$$

Toujours dans ce cas, on vérifie $w_{X, k \tau}(f)=k w_{X, \tau}(f)$ pour tout $k \in \mathbf{N}^{\times}$et $w_{X, \tau+\tau^{\prime}(1, \ldots, 1)}(f)=$ $w_{X, \tau}(f)-\tau^{\prime} \operatorname{deg}(H)$ pour $\tau^{\prime} \in \mathbf{N}$. On peut donc écrire en général (c'est-à-dire pour $\tau \in \mathbf{R}^{N+1}$ ) l'égalité

en posant

$$
e_{\tau}(X \cdot H)=e_{\tau}(X) \operatorname{deg}(H)+w_{X, \tau}(f) \operatorname{deg}(X)
$$

$$
w_{X, \tau}(f):=\lim _{k \rightarrow \infty} \frac{1}{k} w_{X,\left[k\left(\tau-\tau^{\prime}(1, \ldots, 1)\right)\right]}(f)+\tau^{\prime} \operatorname{deg}(H)
$$


où $\tau^{\prime}:=\min \left(\tau_{0}, \ldots, \tau_{N}\right)$ et $[\cdot]$ désigne le vecteur des parties entières dans $\mathbf{N}^{N+1}$.

Dans le cas d'une variété torique $X_{\mathcal{A}, \alpha}$ le poids de Chow $e_{\tau}\left(X_{\mathcal{A}, \alpha}\right)=e_{\tau}\left(X_{\mathcal{A}}\right)$ s'exprime naturellement en termes d'un polytope construit à l'aide du vecteur $\tau$, au-dessus du polytope $Q_{\mathcal{A}}$ déjà introduit au paragraphe \.

Proposition IV.3. - (PS04 Prop. III.1]) - Avec les notations introduites on suppose $\mathbf{Z} a_{0}+\cdots+\mathbf{Z} a_{N}=\mathbf{Z}^{n}$. Soit $Q_{\mathcal{A}, \tau}$ l'enveloppe convexe des points $\left(a_{0}, \tau_{0}\right), \ldots,\left(a_{N}, \tau_{N}\right)$ dans $\mathbf{R}^{n+1}$ et $\vartheta_{\mathcal{A}, \tau}: Q_{\mathcal{A}} \rightarrow \mathbf{R}$ la paramétrisation de la toiture de $Q_{\mathcal{A}, \tau}$ au-dessus de $Q_{\mathcal{A}}$, alors

$$
e_{\tau}\left(X_{\mathcal{A}}\right)=(n+1) ! \int_{Q_{\mathcal{A}}} \vartheta_{\mathcal{A}, \tau}(u) d u_{1} \ldots d u_{n} .
$$

La démonstration qu'on donne de cette proposition dans [PS04 § III] est basée sur un calcul explicite des poids de Hilbert des variétés toriques; nous y montrons également qu'on peut l'obtenir comme conséquence des résultats de I.M. Gelfand, M.M. Kapranov et A.V. Zelevinski sur le polytope de Newton du $\mathcal{A}$-résultant GKZ94, Ch. 7 et 8]. Elle est également implicite dans Don02 § 4.2]. L'interprétation ci-dessus du poids de Chow comme bi-degré permet d'en donner encore une autre démonstration simple et directe :

Démonstration. - Tout d'abord on remarque qu'il suffit de démontrer l'énoncé pour un choix générique (au sens de Zariski) du vecteur $\tau$ dans $\mathbf{R}^{N+1}$, puisque les termes considérés sont continus par rapport à $\tau$. L'identité étant invariante par homothéties et translations, on peut se ramener à supposer $\tau \in \mathbf{N}^{N+1}$ à coordonnées premières entre elles dans leur ensemble.

On a $L_{\mathcal{A}, \tau}=\mathbf{Z}^{n+1}$; la proposition $\amalg .2$ entraîne alors

$$
e_{\tau}(X)=\operatorname{deg}_{(0, n+1)}\left(\widetilde{X}_{\tau}\right)=\operatorname{MV}_{n+1}\left(Q_{\mathcal{A}, \widetilde{\tau}}, \ldots, Q_{\mathcal{A}, \widetilde{\tau}}\right)=(n+1) ! \int_{Q_{\mathcal{A}}} \vartheta_{\mathcal{A}, \tau}(u) d u_{1} \cdots d u_{n}
$$

où $\widetilde{\tau}=\left(\tau_{0}, \ldots, \tau_{N}, 0, \ldots, 0\right) \in \mathbf{N}^{2 N+2}$.

La proposition IV.3 permet de reformuler de façon amusante le théorème du sous-espace de Schmidt, dans la version projective qu'en ont donné Evertse et Ferretti [EF02] mais dans le cas très particulier des variétés toriques.

Convention IV.4. - Si $K$ est un corps de nombres et $v$ une place de $K$ on notera $|\cdot|_{v}$ la valeur absolue de $K$ dans $v$ étendant la valeur absolue usuelle de $\mathbf{Q}$ si $v$ est archimédienne et la valeur absolue $p$-adique standard de $\mathbf{Q}$ (i.e. $|p|_{v}=p^{-1}$ ) si v est ultramétrique. On notera encore $M_{K}$ l'ensemble de ces valeurs absolues représentant les places de $K$.

Soit $K$ un corps de nombres et $S$ un ensemble fini de places de $K$. On considère une variété torique $X_{\mathcal{A}, \alpha} \subset \mathbf{P}_{N}$ définie sur $K$, pour toute place $v \in S$ des réels $\tau_{v, 0}, \ldots, \tau_{v, N} \geq 0$ et le système d'inéquations en $x \in X_{\mathcal{A}, \alpha}(\overline{\mathbf{Q}})$ :

(IV.10) $\log \left(\frac{\left|x_{i}\right|_{w}}{\|x\|_{w}}\right) \leq-\tau_{v, i} . h(x) \quad$ pour tout $i=0, \ldots, N$ et toute place $w \mid v$ de $K(x)$,

où $\|x\|_{w}:=\max \left(\left|x_{i}\right|_{w} ; i=0, \ldots, N\right)$ si $w$ est ultramétrique, $\|x\|_{w}=\left(\sum_{i=0}^{N}\left|x_{i}\right|_{w}^{2}\right)^{1 / 2}$ si $w$ est archimédienne, et $h(x)$ désigne la hauteur projective.

Dans cette situation notons $\vartheta_{\mathcal{A}, \tau}(u):=\sum_{v \in S} \frac{\left[K_{v}: \mathbf{Q}_{v}\right]}{[K: \mathbf{Q}]} \cdot \vartheta_{\mathcal{A}, \tau_{v}}(u)$ pour $u \in Q_{\mathcal{A}}$. Le théorème 3.2 de [EF02] explicite pour tout $\varepsilon>0$ des réels

$$
c_{1}\left(N, n, \operatorname{deg}\left(X_{\mathcal{A}, \alpha}\right), \varepsilon\right), \quad c_{2}\left(N, n, \operatorname{deg}\left(X_{\mathcal{A}, \alpha}\right), \varepsilon\right) \geq 1
$$


tels que l'énoncé suivant soit vrai $\left(\tau_{v}:=\left(\tau_{v, 0}, \ldots, \tau_{v, N}\right), v \in S\right)$ :

Théorème IV.5. - (EF02, Thm. 3.2) - Si la valeur moyenne de $\vartheta_{\mathcal{A}, \tau}$ sur $Q_{\mathcal{A}}$ est $\geq 1+\varepsilon$, alors les points $x \in X_{\mathcal{A}, \alpha}(\overline{\mathbf{Q}})$ solutions du système d'inéquations (IV.10) et satisfaisant

$$
h(x) \geq c_{1}\left(N, n, \operatorname{deg}\left(X_{\mathcal{A}, \alpha}\right), \varepsilon\right) \cdot\left(1+h\left(X_{\mathcal{A}, \alpha}\right)\right)
$$

appartiennent à un sous-ensemble algébrique propre de $X_{\mathcal{A}, \alpha}$, défini sur $K$ et de degré $\leq$ $c_{2}\left(N, n, \operatorname{deg}\left(X_{\mathcal{A}, \alpha}\right), \varepsilon\right)$.

Démonstration. - Notons $X=X_{\mathcal{A}, \alpha}$, la condition donnée dans le théorème 3.2 de [EF02 s'écrit

$$
\frac{1}{(n+1) \operatorname{deg}(X)} \cdot \sum_{v \in S} \frac{\left[K_{v}: \mathbf{Q}_{v}\right]}{[K: \mathbf{Q}]} \cdot e_{\tau_{v}}(X) \geq 1+\varepsilon .
$$

Or $\operatorname{deg}(X)=n ! \operatorname{Vol}_{n}\left(Q_{\mathcal{A}}\right)$ d'après la formule (I.1) et, vue la proposition IV.3. on a

$$
\sum_{v \in S} \frac{\left[K_{v}: \mathbf{Q}_{v}\right]}{[K: \mathbf{Q}]} \cdot \frac{e_{\tau_{v}}(X)}{(n+1) \operatorname{deg}(X)}=\frac{1}{\operatorname{Vol}_{n}\left(Q_{\mathcal{A}}\right)} \cdot \int_{Q_{\mathcal{A}}} \vartheta_{\mathcal{A}, \tau}(u) d u_{1} \ldots d u_{n}
$$

qui est bien la valeur moyenne de $\vartheta_{\mathcal{A}, \tau}$ au-dessus $Q_{\mathcal{A}}$.

Le résultat trivial, provenant de la formule du produit, permet d'affirmer que le sytème (IV.10) n'a pas de solution dans $\mathbf{P}_{N}^{\circ}(\overline{\mathbf{Q}})$ dès qu'il existe $i \in\{0, \ldots, N\}$ tel que

$$
\sum_{v \in S} \frac{\left[K_{v}: \mathbf{Q}_{v}\right]}{[K: \mathbf{Q}]} \cdot \tau_{v, i}>1 \text {. }
$$

On donne un exemple où ce résultat trivial s'applique bien que la condition du théorème IV.5 ne soit pas remplie et un autre exemple où le théorème IV.5 donne un résultat non trivial.

Exemple IV.6. - Soient $0<\varepsilon<n+1, \mathcal{A} \subset \mathbf{Z}^{n}$ et $\tau_{v}$ tels que $\tau_{v, i}=0$ pour tout $v \in S$ et $i=1, \ldots, N$ et $\sum_{v \in S} \frac{\left[K_{v}: \mathbf{Q}_{v}\right]}{[K: \mathbf{Q}]} \tau_{v, 0}=1+\varepsilon>1$. On calcule à l'aide de la proposition IV.3 $e_{\tau_{v}}\left(X_{\mathcal{A}}\right) \leq \operatorname{deg}\left(X_{\mathcal{A}}\right) \cdot \tau_{v, 0}$, d'où $\sum_{v \in S} \frac{\left[K_{v}: \mathbf{Q}_{v}\right]}{[K: \mathbf{Q}]} \cdot \frac{e_{\tau_{v}}\left(X_{\mathcal{A}}\right)}{(n+1) \operatorname{deg}\left(X_{\mathcal{A}}\right)} \leq \frac{1+\varepsilon}{n+1}<1$. On sait donc que dans ce cas le système d'inéquations (IV.10) n'a pas de solution telle que $x_{0} \neq 0$, bien que l'hypothèse principale du théorème IV.5 ne soit pas satisfaite.

Exemple IV.7. - Soit maintenant $N^{\prime}>3$ un entier, $N:=2 N^{\prime}$ et $D>N^{\prime}\left(N^{\prime}-1\right)$ un autre entier. On considère $\mathcal{A}:=\left(0, \ldots, N^{\prime}-1, D-N^{\prime}+1, \ldots, D\right) \in \mathbf{Z}$ et $S=\left\{v_{1}, \ldots, v_{N^{\prime}}\right\}$ un ensemble de $N^{\prime}$ places de $\mathbf{Q}$. À chaque place $v_{i}$ de $S$ on associe le vecteur $\tau_{v_{i}} \in \mathbf{R}^{N+1}$ dont toutes les coordonnées sont nulles sauf celles d'indices $i$ et $D-N^{\prime}+i$ qui valent $\frac{1}{N^{\prime}-1}$. On a ainsi $e_{\tau_{v_{i}}}\left(X_{\mathcal{A}}\right) \geq 2 \frac{D-N^{\prime}+1}{N^{\prime}-1}$ et $\sum_{v \in S} \frac{e_{\tau_{v}}\left(X_{\mathcal{A}}\right)}{2 \operatorname{deg}\left(X_{\mathcal{A}}\right)} \geq \frac{D-N^{\prime}+1}{D} \cdot \frac{N^{\prime}}{N^{\prime}-1}>1$, tandis que $\sum_{v \in S} \tau_{v, i}=$ $\frac{1}{N^{\prime}-1}<1$ pour tout $i=0, \ldots, N$. Le théorème IV.5 s'applique donc et donne un résultat a priori non trivial dans ce cas.

\section{Hauteur normalisée}

L'espace projectif peut être vu comme une compactification équivariante du groupe multiplicatif $\mathbf{G}_{m}^{N} \simeq\left(\mathbf{P}^{N}\right)^{\circ}$, cette structure de groupe permettant de définir une notion de hauteur pour les sous-variétés de $\mathbf{P}^{N}$ plus canonique que les autres, appelée hauteur normalisée. Cette notion joue un rôle central dans l'approximation diophantienne sur les tores, et tout particulièrement dans les problèmes de Lehmer généralisé et de Bogomolov sur les tores, voir DP99], AD03 et leurs références, voir également Dav03 pour un aperçu historique. 
Suivant DP99, la hauteur normalisée peut se définir par un procédé «à la Tate ». De façon précise, pour $k \in \mathbf{N}$ on pose $[k]: \mathbf{P}^{N} \rightarrow \mathbf{P}^{N},\left(x_{0}: \cdots: x_{N}\right) \mapsto\left(x_{0}^{k}: \cdots: x_{N}^{k}\right)$ l'application puissance $k$-ième; restreinte au tore $\left(\mathbf{P}^{N}\right)^{\circ}$ c'est l'application de multiplication par $k$. La hauteur normalisée d'une variété projective $X$ est par définition

$$
\widehat{h}(X):=\operatorname{deg}(X) \cdot \lim _{k \rightarrow \infty} \frac{h([k] X)}{k \operatorname{deg}([k] X)} \in \mathbf{R}_{+},
$$

où deg et $h$ désignent le degré et la hauteur projective, voir [DP99, § 2] ou [PS04 § I.2]. Lorsque $X$ est de dimension 0, il s'agit de la hauteur de Gauss-Weil des points.

On sait que le comportement de cette hauteur normalisée est lié à la nature géométrique de la variété $X$. En particulier, $\widehat{h}(X)=0$ si et seulement si $X$ est une variété de torsion, c'est-à-dire un translaté de sous-tore un point de torsion. Dans [PS04] on a établi un analogue arithmétique de l'expression du degré d'une variété torique comme volume du polytope associé, que nous rappelons maintenant.

Soit $M_{K}$ l'ensemble des places du corps $K$ et rappelons la convention IV.4 Pour chaque $v \in M_{K}$ on considère le vecteur $\tau_{\alpha v}:=\left(\log \left|\alpha_{0}\right|_{v}, \ldots, \log \left|\alpha_{N}\right|_{v}\right) \in \mathbf{R}^{N+1}$ et le polytope

$$
Q_{\mathcal{A}, \tau_{\alpha v}}:=\operatorname{Conv}\left(\left(a_{0}, \log \left|\alpha_{0}\right|_{v}\right), \ldots,\left(a_{N}, \log \left|\alpha_{N}\right|_{v}\right)\right) \subset \mathbf{R}^{n+1},
$$

dont la toiture au-dessus de $Q_{\mathcal{A}}$ (c'est-à-dire l'enveloppe supérieure) s'envoie bijectivement sur $Q_{\mathcal{A}}$ par la projection standard $\mathbf{R}^{n+1} \rightarrow \mathbf{R}^{n}$. On pose alors

$$
\vartheta_{\mathcal{A}, \tau_{\alpha v}}: Q_{\mathcal{A}} \rightarrow \mathbf{R}, \quad x \mapsto \max \left\{y \in \mathbf{R}:(x, y) \in Q_{\mathcal{A}, \tau_{\alpha v}}\right\}
$$

la paramétrisation de cette toiture; c'est une fonction concave et affine par morceaux.

Théorème V.1. - (PS04, Thm. 0.1]) - Soit $\mathcal{A} \in\left(\mathbf{Z}^{n}\right)^{N+1}$ tel que $L_{\mathcal{A}}=\mathbf{Z}^{n}$ et $\alpha \in$ $\left(K^{\times}\right)^{N+1}$, alors

$$
\widehat{h}\left(X_{\mathcal{A}, \alpha}\right)=\sum_{v \in M_{K}} \frac{\left[K_{v}: \mathbf{Q}_{v}\right]}{[K: \mathbf{Q}]} e_{\tau_{\alpha v}}\left(X_{\mathcal{A}}\right)=(n+1) ! \sum_{v \in M_{K}} \frac{\left[K_{v}: \mathbf{Q}_{v}\right]}{[K: \mathbf{Q}]} \int_{Q_{\mathcal{A}}} \vartheta_{\mathcal{A}, \tau_{\alpha v}}(u) d u_{1} \cdots d u_{n}
$$

Notons que $\tau_{\alpha v}=0$ pour presque tout $v$, donc cette somme ne contient qu'un nombre fini de termes non nuls. Dans les cas des points $(n=0)$ la formule se réduit à la définition usuelle de la hauteur normalisée (hauteur de Gauss-Weil) d'un point de $\mathbf{P}^{N}$.

Comme on a $\widehat{h}\left(X_{\mathcal{A}}\right)=0$, on peut s'interroger si cette formule n'est pas la manifestation d'une propriété générale de la hauteur normalisée par translation. Dans ce sens, B. Sturmfels nous a demandé si pour toute variété projective $X$ on a

$$
\widehat{h}(\alpha X)=\widehat{h}(X)+\sum_{v \in M_{K}} \frac{\left[K_{v}: \mathbf{Q}_{v}\right]}{[K: \mathbf{Q}]} e_{\tau_{\alpha v}}(X) ?
$$

Soit $\prod_{i=0}^{n} \prod_{j=0}^{N} U_{i, j}^{b_{i, j}}$ un monôme apparaissant dans la forme de Chow de $X$, on vérifie facilement

$$
e_{\tau_{\alpha v}}(X) \geq\left(\sum_{i=0}^{n} b_{i, 0}\right) \tau_{\alpha v, 0}+\cdots+\left(\sum_{i=0}^{n} b_{i, N}\right) \tau_{\alpha v, N}
$$

Comme $\sum_{v \in M_{K}} \frac{\left[K_{v}: \mathbf{Q}_{v}\right]}{[K: \mathbf{Q}]} \tau_{\alpha v, i}=0$ pour tout $i=0, \ldots, N$ par la formule du produit, on en déduit

$$
\sum_{v \in M_{K}} \frac{\left[K_{v}: \mathbf{Q} v\right]}{[K: \mathbf{Q}]} e_{\tau_{\alpha v}}(X) \geq 0 \quad \text { pour tout } \alpha \in\left(\overline{\mathbf{Q}}^{\times}\right)^{N+1}
$$


Une formule du type précédent ne peut donc être valable en général.

Illustrons cette formule par un exemple en dimension 1. Soient $\mathcal{A}_{N}:=(0,1,2, \ldots, N) \in$ $\mathbf{Z}^{N+1}$ et $\alpha_{N}:=(1,2,3, \ldots, N+1) \in\left(\overline{\mathbf{Q}}^{\times}\right)^{N+1} ;$ ainsi

$$
\varphi_{\mathcal{A}_{N}, \alpha_{N}}: \mathbf{G}_{m} \rightarrow \mathbf{P}^{N} \quad, \quad s \mapsto\left(1: 2 s: \cdots:(N+1) s^{N}\right) .
$$

Les figures suivantes montrent pour $N=3$ les polytopes associés et leurs toitures, pour chaque place $v \in M_{\mathbf{Q}}$ :
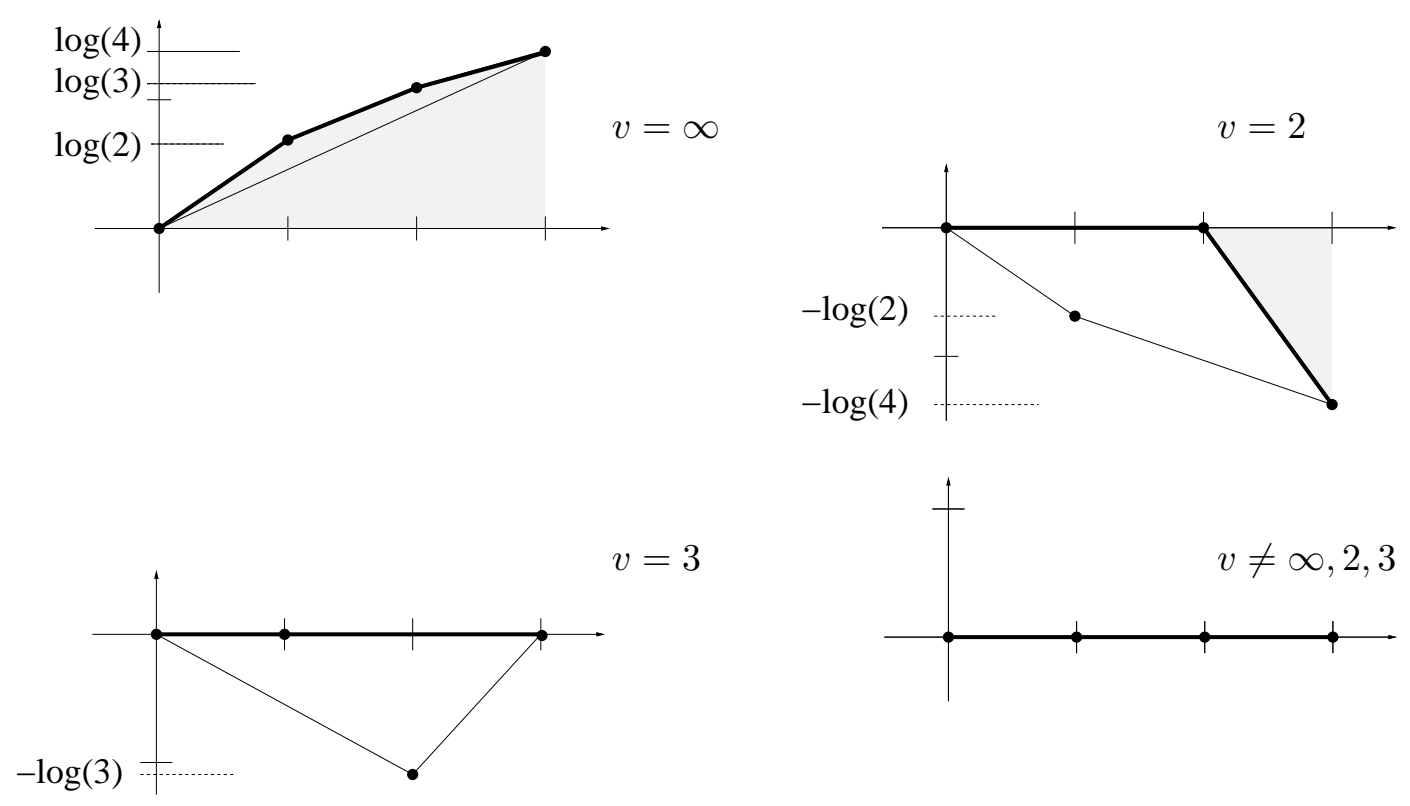

Ainsi $\vartheta_{v} \equiv 0$ pour $v \neq \infty, 2$, d'où

$$
\widehat{h}\left(X_{\mathcal{A}_{3}, \alpha_{3}}\right)=2 !\left(\int_{0}^{3} \vartheta_{\infty}(u) d u+\int_{0}^{3} \vartheta_{2}(u) d u\right)=2 \log (2)+2 \log (3)=\log (36) .
$$

En général

$$
\begin{aligned}
\widehat{h}\left(X_{\mathcal{A}_{N}, \alpha_{N}}\right) & =2 !\left(\int_{0}^{N} \vartheta_{\infty}(u) d u+\sum_{p \mid N+1} \int_{0}^{N} \vartheta_{p}(u) d u\right) \\
& =2\left(\log (2)+\cdots+\log (N)+\frac{1}{2} \log (N+1)+\sum_{p \mid N+1} \frac{1}{2} \log |N+1|_{p}\right) \\
& =2 \log (N !) .
\end{aligned}
$$

À l'instar des multidegrés, les multihauteurs du tore $\mathbf{G}_{m}^{n}$ plongé dans un produit d'espaces projectifs via plusieurs applications monomiales peuvent aussi s'expliciter à l'aide d'intégrales mixtes des fonctions concaves apparaissant dans le théorème $\mathbf{D . 1}$

Soit $Z \subset \mathbf{P}^{N_{0}} \times \cdots \times \mathbf{P}^{N_{m}}$ de dimension $n$ et $c=\left(c_{0}, \ldots, c_{m}\right) \in \mathbf{N}_{n+1}^{m+1}$ avec $0 \leq c_{i} \leq N_{i}$. Soit rés $\left._{d(c)}(I(Z))\right)$ la forme résultante associée, dans les notations du $\S$ 【 La multihauteur projective de $Z$ d'indice $c$ est définie par

$$
h_{c}(Z):=h\left(\operatorname{rés}_{d(c)}(I(Z))\right) \text {, }
$$

où $h$ désigne la hauteur des polynômes multihomogènes définie à l'aide de la $S_{N_{0}+1} \times \cdots \times$ $S_{N_{n}+1}$-mesure pour les places archimediennes; on renvoie à [Rem01a ou encore [PS04, $\S$ I.2] 
pour les détails. Notons $s: \mathbf{P}^{N_{0}} \times \cdots \times \mathbf{P}^{N_{m}} \rightarrow \mathbf{P}^{\left(N_{0}+1\right) \cdots\left(N_{m}+1\right)-1}$ le plongement de Segre, la suite

$$
k \mapsto \operatorname{deg}(s(Z)) \cdot \frac{h_{c}([k] Z)}{k \operatorname{deg}([k] s(Z))}
$$

converge lorsque $k$ tend vers l'infini [PS04, Prop. I.2]). Sa limite est par définition la multihauteur normalisée de $Z$ d'indice $c$, notée $\widehat{h}_{c}(Z)$.

Pour $D=\left(D_{0}, \ldots, D_{m}\right) \in\left(\mathbf{N}^{\times}\right)^{m+1}$ soit $\Psi_{D}: \mathbf{P}^{N_{0}} \times \cdots \times \mathbf{P}^{N_{m}} \rightarrow \mathbf{P}^{\left(\begin{array}{c}D_{0}+N_{0} \\ N_{0}\end{array}\right) \cdots\left(\begin{array}{c}D_{m}+N_{m} \\ N_{m}\end{array}\right)-1}$ le plongement mixte introduit au paragraphe \alors on a la formule [PS04, (I.4)]

$$
\widehat{h}\left(\Psi_{D}(Z)\right)=\sum_{c \in \mathbf{N}_{n+1}^{m+1}}\left(\begin{array}{c}
n+1 \\
c
\end{array}\right) \widehat{h}_{c}(Z) D^{c} .
$$

Les multihauteurs des variétés toriques s'explicitent en termes de ce qu'on appelle des intégrales mixtes d'une famille de fonctions concaves [PS04 § IV.3], notion analogue au volume mixte. Soient $f: Q \rightarrow \mathbf{R}$ et $g: R \rightarrow \mathbf{R}$ des fonctions concaves définies sur des ensembles convexes $Q, R \subset \mathbf{R}^{n}$ respectivement. On pose

$$
f \boxplus g: Q+R \rightarrow \mathbf{R}, \quad x \mapsto \max \{f(y)+g(z): y \in Q, z \in R, y+z=x\},
$$

qui est une fonction concave définie sur la somme de Minkowski $Q+R$; on obtient ainsi une structure de semi-groupe commutatif sur l'ensemble des fonctions concaves (définies sur des ensembles convexes). Pour une famille de fonctions concaves $f_{0}: Q_{0} \rightarrow \mathbf{R}, \ldots, f_{n}: Q_{n} \rightarrow \mathbf{R}$ l'intégrale mixte (ou multi-intégrale) est définie via la formule

$$
\operatorname{MI}\left(f_{0}, \ldots, f_{n}\right):=\sum_{j=0}^{n}(-1)^{n-j} \sum_{0 \leq i_{0}<\cdots<i_{j} \leq n} \int_{Q_{i_{0}}+\cdots+Q_{i_{j}}}\left(f_{i_{0}} \boxplus \cdots \boxplus f_{i_{j}}\right)(u) d u_{1} \cdots d u_{n} .
$$

Comme pour le volume mixte, l'intégrale mixte est une fonctionnelle positive, symétrique et linéaire en chaque variable $f_{i}$, voir [PS04, $\S$ IV.3].

Soit $\mathcal{A}_{0} \in\left(\mathbf{Z}^{n}\right)^{N_{0}+1}, \ldots, \mathcal{A}_{m} \in\left(\mathbf{Z}^{n}\right)^{N_{m}+1}$ tels que $L_{\mathcal{A}_{0}}+\cdots+L_{\mathcal{A}_{m}}=\mathbf{Z}^{n}$ et $\underline{\mathcal{A}}:=$ $\left(\mathcal{A}_{0}, \ldots, \mathcal{A}_{m}\right)$. Soit $\alpha_{0} \in\left(K^{\times}\right)^{N_{0}+1}, \ldots, \alpha_{m} \in\left(K^{\times}\right)^{N_{m}+1}$ et posons $\underline{\alpha}:=\left(\alpha_{0}, \ldots, \alpha_{m}\right)$. Considérons alors l'action monomiale $*_{\mathcal{A}}$ de $\mathbf{G}_{m}^{n}$ sur le produit d'espaces projectifs $\mathbf{P}^{N_{0}} \times \cdots \times \mathbf{P}^{N_{m}}$ associée; on note $X_{\underline{\mathcal{A}}, \underline{\alpha}}$ l'adhérence de Zariski de l'orbite du point $\underline{\alpha} \in \mathbf{P}^{N_{0}} \times \cdots \times \mathbf{P}^{N_{m}}$.

Pour chaque $v \in \bar{M}_{K}$ on note aussi $\vartheta_{\mathcal{A}_{i}, \tau_{\alpha_{i} v}}: Q_{\mathcal{A}_{i}} \rightarrow \mathbf{R}$ la fonction paramétrant la toiture du polytope $Q_{\mathcal{A}_{i}, \tau_{\alpha_{i} v}} \subset \mathbf{R}^{n+1}$ associé au vecteur $\mathcal{A}_{i}$ et au poids $\tau_{\alpha_{i} v}$.

Théorème V.2. - PS04, Thm. 0.3 et Rem. IV.7] - Soit $c \in \mathbf{N}_{n+1}^{m+1}$, dans la situation ci-dessus on a

$$
\widehat{h}_{c}\left(X_{\underline{\mathcal{A}}, \underline{\alpha}}\right)=\sum_{v \in M_{K}} \frac{\left[K_{v}: \mathbf{Q}_{v}\right]}{[K: \mathbf{Q}]} \operatorname{MI}_{c}\left(\vartheta_{\underline{\mathcal{A}}, \tau_{\underline{\alpha}} v}\right)
$$

$\operatorname{avec} \operatorname{MI}_{c}\left(\vartheta_{\underline{\mathcal{A}}, \tau_{\underline{\alpha} v}}\right):=\operatorname{MI}(\underbrace{\vartheta_{\mathcal{A}_{0}, \tau_{\alpha_{0} v}, \ldots, \vartheta_{\mathcal{A}_{0}, \tau_{\alpha_{0} v}}}}_{c_{0} \text { fois }}, \ldots, \underbrace{\vartheta_{\mathcal{A}_{m}, \tau_{\alpha_{m} v}, \ldots, \vartheta_{\mathcal{A}_{m}, \tau_{\alpha_{m} v}}}}_{c_{m} \text { fois }})$.

Exemple V.3. - Soient $\xi_{1}, \ldots, \xi_{N} \in K^{\times}$et considérons l'application monomiale

$$
\begin{aligned}
& \mathbf{G}_{m} \stackrel{\varphi}{\longrightarrow} \quad\left(\mathbf{P}^{1}\right)^{N} \quad \stackrel{\text { Segre }}{\longrightarrow} \mathbf{P}^{2^{N}-1} \\
& s \longmapsto\left(\left(1: \xi_{1} s\right), \ldots,\left(1: \xi_{N} s\right)\right) \longmapsto\left(\left(\prod_{j \in J} \xi_{j}\right) \cdot s^{\operatorname{Card}(J)}: J \subset\{1, \ldots, N\}\right) .
\end{aligned}
$$


Soit $X$ l'image de $\varphi$ dans $\left(\mathbf{P}^{1}\right)^{N}$ et pour $1 \leq i, j \leq N$ notons $c(i, j) \in \mathbf{N}^{N}$ le vecteur dont les coordonnées d'indices $i$ et $j$ valent 1 et les autres 0 , on vérifie à l'aide du théorème $\nabla . \mathbb{Q}$ et de la définition des multi-intégrales

$$
\begin{aligned}
\widehat{h}_{c(i, j)}(X) & =\sum_{v \in M_{K}} \frac{\left[K_{v}: \mathbf{Q}_{v}\right]}{[K: \mathbf{Q}]} \operatorname{MI}_{c}\left(\vartheta_{\underline{\mathcal{A}}, \tau_{\underline{\alpha}}}\right) \\
& =\sum_{v \in M_{K}} \frac{\left[K_{v}: \mathbf{Q}_{v}\right]}{[K: \mathbf{Q}]} \max \left(\log \left|\xi_{i}\right|_{v} ; \log \left|\xi_{j}\right|_{v}\right) \\
& =h\left(\xi_{i}: \xi_{j}\right) .
\end{aligned}
$$

Dans la figure suivante les parties grisées montrent pour $N=2$ et en supposant $\log \left|\xi_{i}\right| \leq$ $\log \left|\xi_{j}\right|$, pour chaque cas le calcul de la multi-intégrale $\operatorname{MI}_{c}\left(\vartheta_{\underline{\mathcal{A}}, \tau_{\underline{\alpha}}}\right)$.
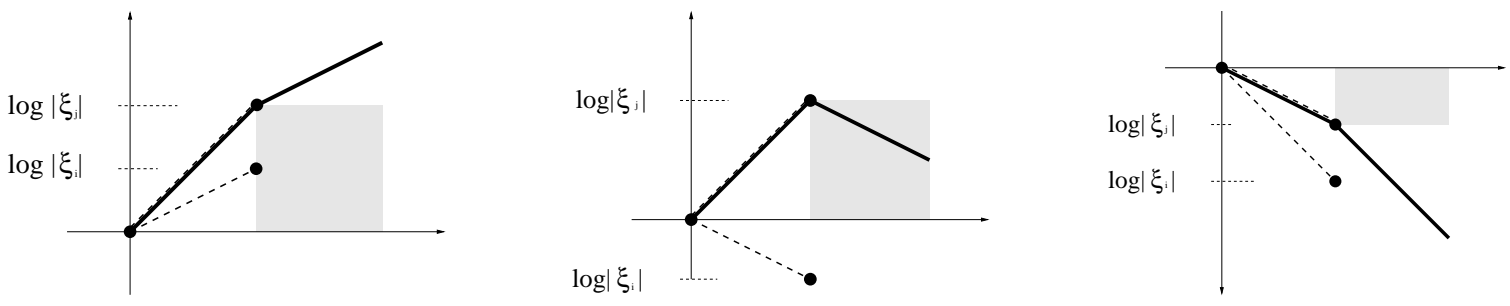

Par la formule (V.11), on en déduit que la hauteur de l'image de Segre $\odot \varphi$ dans $\mathbf{P}^{2^{N}-1}$ est égale à

$$
\widehat{h}(\operatorname{Segre}(X))=2 \sum_{1 \leq i<j \leq N} \widehat{h}_{c(i, j)}(X)=2 \sum_{1 \leq i<j \leq N} h\left(\left(\xi_{i}: \xi_{j}\right)\right)=\sum_{1 \leq i, j \leq N} h\left(\left(\xi_{i}: \xi_{j}\right)\right) .
$$

Une conséquence du théorème $\nabla .1$ est que $\widehat{h}\left(X_{\mathcal{A}, \alpha}\right)$ est le logarithme d'un nombre algébrique. En effet, $e_{\tau_{\alpha} v}\left(X_{\mathcal{A}}\right)=0$ pour toutes les places $v$ sauf un nombre fini et, comme le poids de Chow est combinaison à coefficients entiers des composantes du vecteur poids $\tau_{\alpha v}$, c'est à dire des $\log |\alpha|_{v}$, notre assertion est claire. Un tel logarithme de nombre algébrique étant nul ou transcendant, on peut énoncer:

Proposition V.4. - Soit $X$ une variété torique qui n'est pas de torsion, alors $\widehat{h}(X) \notin \overline{\mathbf{Q}}$.

Les hauteurs normalisées des variétés toriques qui ne sont pas de torsion, sont donc des nombres transcendants parmi les plus simples que l'on connaisse, à savoir les logarithmes de nombres algébriques. On sait qu'il devrait résulter des conjectures les plus générales sur les variétés et les motifs que les hauteurs des variétés projectives s'expriment rationnellement en termes de valeurs de fonctions $L$ et de leurs dérivées, ou encore en termes de polylogarithmes.

Dès qu'on considère des variétés projectives qui ne sont plus toriques on doit s'attendre à ce que leur hauteur normalisée fasse effectivement intervenir des polylogarithmes supérieurs. De même, si l'on s'intéresse à la hauteur projective d'une variété torique, une quantité supplémentaire s'ajoute aux intégrales des fonctions $\vartheta_{\mathcal{A}, \tau_{\alpha} v}$, qui s'écrit bien sur des exemples à l'aide de polylogarithmes mais est en général difficile à évaluer exactement (calculs non publiés).

\section{Optimalité du théorème des minimums algébriques successifs}

Dans ce paragraphe on étudie les minimums algébriques successifs des variétés toriques, définis dans l'introduction. Comme résultat principal, on démontre le théorème 0.4 énoncé dans l'introduction qui entraîne l'optimalité des estimations dans le théorème des minimums algébriques successifs: on construit des exemples montrant qu'à des $\varepsilon$-près, toute configuration 
possible des minimums successifs se réalise et que le quotient $\widehat{h}(X) / \operatorname{deg}(X)$ peut atteindre n'importe quel valeur dans l'intervalle autorisé par les inégalités (II).

Notons que l'analogue abélien du théorème 0.4 est faux: soit $A$ une variété abélienne définie sur $\overline{\mathbf{Q}}$ munie d'un fibré en droites $L$ ample et symétrique, permettant de définir une notion de hauteur normalisé $\widehat{h}=\widehat{h}_{L}$ pour les sous-variétés de $A$ Phi91. En particulier, on a une notion de minimums successifs et le théorème de Zhang y est toujours valable, voir Zha95. Thm. 5.2].

Soit $\alpha+B \subset A$ le translaté d'une sous-variété abélienne $B$ par un point $\alpha$ et $\operatorname{Tors}(B)$ le sousgroupe des points de torsion de $B$. Soit $\beta$ un point quelconque dans $\alpha+B$, alors $\beta+\operatorname{Tors}(B)$ est un sous-ensemble de points de hauteur $\widehat{h}(\beta)$ dense dans $\alpha+B$ (puisque Tors $(B)$ est Zariski dense dans $B)$, donc $\widehat{\mu}^{\text {ess }}(\alpha+B) \leq \widehat{h}(\beta)$. On en déduit $\widehat{\mu}^{\text {ess }}(\alpha+B)=\widehat{\mu}^{\text {abs }}(\alpha+B)$ et donc

$$
\widehat{\mu}_{i}(\alpha+B)=\widehat{\mu}^{\text {ess }}(\alpha+B) \quad \text { pour } i=1, \ldots, n+1 .
$$

Ainsi, dans cette situation l'intervalle du théorème des minimums successifs se réduit à un point, et on a les égalités

$$
\frac{\widehat{h}(\alpha+B)}{\operatorname{deg}(\alpha+B)}=(n+1) \widehat{\mu}^{\mathrm{ess}}(\alpha+B)=\widehat{\mu}_{1}(\alpha+B)+\cdots+\widehat{\mu}_{n+1}(\alpha+B) .
$$

La situation est plus riche dans le cas torique, la différence tenant au fait que les translatés de sous-tores de $\left(\mathbf{P}^{N}\right)^{\circ}$ ne sont pas des ensembles fermés.

Soit $\mathcal{A}=\left(a_{0}, \ldots, a_{N}\right) \in\left(\mathbf{Z}^{n}\right)^{N+1}$ et $\alpha=\left(\alpha_{0}, \ldots, \alpha_{N}\right) \in\left(\overline{\mathbf{Q}}^{\times}\right)^{N+1}$. L'ouvert principal $X_{\mathcal{A}, \alpha}^{\circ}$ est le translaté de sous-tore $\alpha \cdot X_{\mathcal{A}}$ et avec le même raisonnement que pour le cas abélien

$$
\widehat{\mu}^{\mathrm{ess}}\left(X_{\mathcal{A}, \alpha}\right)=\widehat{\mu}^{\mathrm{ess}}\left(X_{\mathcal{A}, \alpha}^{\circ}\right)=\widehat{\mu}_{i}\left(X_{\mathcal{A}, \alpha}^{\circ}\right) \quad \text { pour } i=1, \ldots, n+1 .
$$

Cependant, les autres minimums successifs de $X_{\mathcal{A}, \alpha}$ dépendent des orbites de dimension inférieure, et peuvent donc différer du minimum essentiel.

Lemme VI.1. - Avec les notations ci-dessus, pour $i=1, \ldots, n+1$

$$
\widehat{\mu}_{i}\left(X_{\mathcal{A}, \alpha}\right)=\min \left\{\widehat{\mu}^{\mathrm{abs}}\left(X_{\mathcal{A}, \alpha, P}^{\circ}\right): P \in \mathrm{F}\left(Q_{\mathcal{A}}\right), \operatorname{dim}(P)=n-i+1\right\},
$$

où $P$ parcours l'ensemble des faces $\mathrm{F}\left(Q_{\mathcal{A}}\right)$ du polytope $Q_{\mathcal{A}}$ de dimension $n-i+1$.

Démonstration. - Considérons la décomposition en orbites: $X_{\mathcal{A}, \alpha}=\bigcup_{P \in \mathrm{F}\left(Q_{\mathcal{A}}\right)} X_{\mathcal{A}, \alpha, P}^{\circ}$ (formule ([.3) ). C'est un recouvrement de $X_{\mathcal{A}, \alpha}$ et donc par Som05 Lem. 2.2] on a

$$
\begin{aligned}
\widehat{\mu}_{i}\left(X_{\mathcal{A}}\right) & =\min \left\{\widehat{\mu}_{\operatorname{dim}(P)-n+i}\left(X_{\mathcal{A}, \alpha, P}^{\circ}\right): \operatorname{dim}(P) \geq n-i+1\right\} \\
& =\min \left\{\widehat{\mu}^{\mathrm{abs}}\left(X_{\mathcal{A}, \alpha, P}^{\circ}\right): \operatorname{dim}(P)=n-i+1\right\}
\end{aligned}
$$

car ce minimum est atteint sur les faces de dimension $n-i+1$.

Ainsi, le calcul des minimums successifs de $X_{\mathcal{A}, \alpha}$ se réduit à celui du minimum essentiel (ou absolu) d'un sous-tore. Le lemme suivant donne le minimum essentiel pour certaines variétés toriques particulières.

Lemme VI.2. - Soit $\mathcal{A}=\left(a_{0}, \ldots, a_{N}\right) \in\left(\mathbf{Z}^{n}\right)^{N+1}$ et $\alpha=\left(\alpha_{0}, \ldots, \alpha_{N}\right) \in\left(K^{\times}\right)^{N+1}$, et supposons qu'il existe $a \in \operatorname{Supp}(\mathcal{A})$ tel que pour toute place $v \in M_{K}$ le maximum des $\left|\alpha_{i}\right|_{v}$ pour $i=0, \ldots, N$ est atteint au-dessus de a, autrement-dit

$$
\max \left\{\left|\alpha_{i}\right|_{v}: 0 \leq i \leq N\right\}=\max \left\{\left|\alpha_{\ell}\right|_{v}: 0 \leq \ell \leq N, a_{\ell}=a\right\} .
$$

Alors $\widehat{\mu}^{\mathrm{ess}}\left(X_{\mathcal{A}, \alpha}\right)=\widehat{\mu}^{\mathrm{abs}}\left(X_{\mathcal{A}, \alpha}^{\circ}\right)=\widehat{h}(\alpha)$. 
Démonstration. - Notons $0 \leq \ell_{0}, \ldots, \ell_{M} \leq N$ les indices pour lesquels $a_{\ell}=a$ et soit $\varpi$ : $\mathbf{P}^{N} \rightarrow \mathbf{P}^{M}$ la projection $x \mapsto\left(x_{\ell_{0}}: \cdots: x_{\ell_{M}}\right)$. Alors pour un point quelconque $\xi=\left(\alpha_{0} s^{a_{0}}\right.$ : $\left.\cdots: \alpha_{N} s^{a_{N}}\right) \in X_{\mathcal{A}, \alpha}^{\circ}$ on a $\varpi(\xi)=\left(\alpha_{\ell_{0}} s^{a}: \cdots: \alpha_{\ell_{M}} s^{a}\right)=\left(\alpha_{\ell_{0}}: \cdots: \alpha_{\ell_{M}}\right)$ et donc

$$
\widehat{h}(\xi) \geq \widehat{h}(\varpi(\xi))=\widehat{h}\left(\alpha_{\ell_{0}}: \cdots: \alpha_{\ell_{M}}\right),
$$

et $\widehat{h}\left(\alpha_{\ell_{0}}: \cdots: \alpha_{\ell_{M}}\right)=\widehat{h}(\alpha)$ grâce à l'hypothèse du lemme, donc $\widehat{\mu}^{\text {abs }}\left(X_{\mathcal{A}, \alpha}^{\circ}\right) \geq \widehat{h}(\alpha)$. En outre $\widehat{h}(\alpha) \geq \widehat{\mu}^{\mathrm{ess}}\left(X_{\mathcal{A}, \alpha}\right)$ d'où la conclusion.

Démonstration du théorème 0.4 - On peut supposer sans perte de généralité $N=3 n+1$, puisque le cas général se déduit de celui-ci par immersion de $\mathbf{P}^{3 n+1}$ comme un sous-espace standard de $\mathbf{P}^{N}$.

Soient $d$ un nombre premier, $1 \leq k \leq n, 1 \leq f \leq d-1$ des paramètres entiers à fixer ultérieurement et encore $q_{0} \geq \cdots \geq q_{n} \geq 0$ des paramètres rationnels. Rappelons que $e_{1}, \ldots, e_{n}$ désigne la base standard de $\mathbf{Z}^{n}$ et $S$ le simplexe standard $\operatorname{Conv}\left(0, e_{1}, \ldots, e_{n}\right) \subset \mathbf{R}^{n}$, on pose

$$
\begin{aligned}
& a_{i}:=d e_{i} \\
& b_{i}:= \begin{cases}(d-1) e_{i} & \text { pour } i=1, \ldots, n \\
f e_{i} & \text { pour } 1 \leq i \leq k-1 \\
e_{i} & \text { pour } i=k\end{cases} \\
& \hline
\end{aligned}
$$

puis

$$
\begin{aligned}
& \mathcal{A}:=\left(0, a_{1} \ldots, a_{n}, 0, a_{1}, \ldots, a_{n}, b_{1}, \ldots, b_{n}\right) \in\left(\mathbf{Z}^{n}\right)^{3 n+2}, \\
& \alpha:=(\underbrace{1, \ldots \ldots \ldots \ldots, 1}_{n+1 \text { fois }}, 2^{q_{0}}, 2^{q_{1}}, \ldots \ldots, 2^{q_{n}}, \underbrace{2^{q_{0}}, \ldots, 2^{q_{0}}}_{k \text { fois }}, \underbrace{1, \ldots, 1}_{n-k \text { fois }}) \in\left(\overline{\mathbf{Q}}^{\times}\right)^{3 n+2}
\end{aligned}
$$

et on considère $X \subset \mathbf{P}^{3 n+1}$ la variété torique associée au couple $(\mathcal{A}, \alpha)$ ainsi défini. On a $L_{\mathcal{A}}=\mathbf{Z}^{n}$ et $Q_{\mathcal{A}}=d S$, donc la dimension et le degré de cette variété sont égaux à $n$ et $n ! \operatorname{Vol}_{n}\left(Q_{\mathcal{A}}\right)=d^{n}$ respectivement, et si $\ell$ est un dénominateur commun de $q_{0}, \ldots, q_{n}$, cette variété est définie sur l'extension kummerienne $K:=\mathbf{Q}\left(2^{\frac{1}{\ell}}\right)$. De plus, il résulte du lemmeVI.1 et du lemme VI.2 appliqué à toutes les faces de $Q_{\mathcal{A}}$

$$
\widehat{\mu}_{i}(X)=q_{i-1} \log (2), \quad i=1, \ldots, n+1,
$$

le $i$-ème minimum se réalisant sur la face $\operatorname{Conv}\left(a_{i-1}, \ldots, a_{n}\right)$. En outre, on estime la hauteur en utilisant la formule dans le théorème $\nabla .1$ On décompose le polytope de base en

$$
Q_{\mathcal{A}}=Q_{1} \cup Q_{2} \cup Q_{3}
$$

avec $Q_{1}:=\operatorname{Conv}\left(0, b_{1}, \ldots, b_{k}, a_{k+1}, \ldots, a_{n}\right), Q_{2}:=\operatorname{Conv}\left(b_{1}, \ldots, b_{k}, a_{k}, \ldots, a_{n}\right)$ et $Q_{3}:=Q_{\mathcal{A}} \backslash$ $\left(Q_{1} \cup Q_{2}\right)$. Pour $n=3$ on a une figure du type montré ci-dessous :

Pour toute place finie $v$ de $K$ on vérifie $\vartheta_{\mathcal{A}, \tau_{\alpha v}} \equiv 0$; la formule pour $\widehat{h}(X)$ se réduit alors aux contributions des places archimédiennes. Pour $v \in M_{K}^{\infty}$, la décomposition du domaine considérée sépare l'intégrale en trois morceaux $I_{i}:=\int_{Q_{i}} \vartheta_{\mathcal{A}, \tau_{\alpha v}}(u) d u(i=1,2,3)$. On vérifie d'abord que la fonction $\vartheta_{\mathcal{A}, \tau_{\alpha v}}$ est linéaire sur chacun des simplexes $Q_{1}$ et $Q_{2}$. L'intégrale 

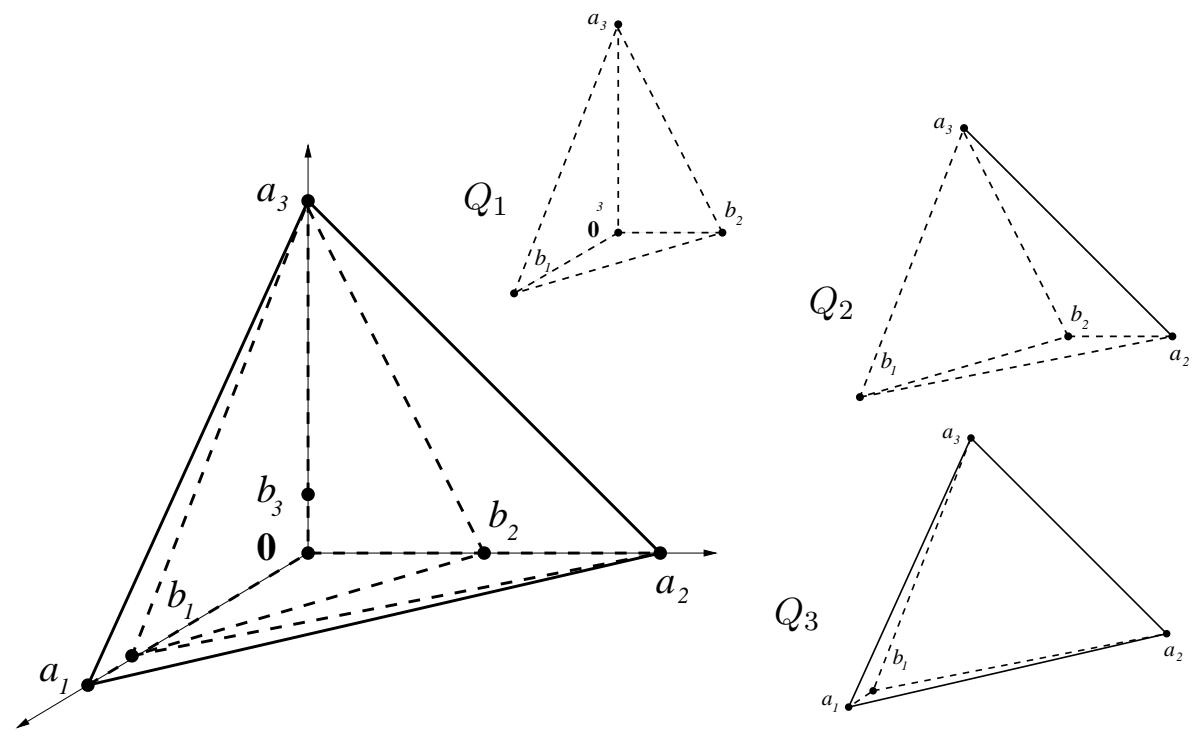

d'une fonction linéaire sur un simplexe étant égale au volume du simplexe multiplié par la valeur moyenne de la fonction, on trouve

$$
\begin{aligned}
I_{1} & =\operatorname{Vol}_{n}\left(Q_{1}\right) \frac{\left((k+1) q_{0}+q_{k+1}+\cdots+q_{n}\right) \log (2)}{n+1} \\
& =\frac{f(d-1)^{k-1} d^{n-k}}{(n+1) !}\left((k+1) q_{0}+q_{k+1}+\cdots+q_{n}\right) \log (2), \\
I_{2} & =\operatorname{Vol}_{n}\left(Q_{2}\right) \frac{\left(k q_{0}+q_{k}+\cdots+q_{n}\right) \log (2)}{n+1} \\
& =\frac{(d-f)(d-1)^{k-1} d^{n-k}}{(n+1) !}\left(k q_{0}+q_{k}+\cdots+q_{n}\right) \log (2) .
\end{aligned}
$$

En outre on estime brutalement la troisième intégrale

$$
0 \leq I_{3} \leq \operatorname{Vol}_{n}\left(Q_{3}\right) \cdot \max \left(\vartheta_{\mathcal{A}, \tau_{\alpha v}}\right)=\frac{d^{n}-(d-1)^{k-1} d^{n-k+1}}{n !} q_{0} \log (2)
$$

puisque $\operatorname{Vol}_{n}\left(Q_{3}\right)=\operatorname{Vol}_{n}\left(Q_{\mathcal{A}}\right)-\operatorname{Vol}_{n}\left(Q_{1} \cup Q_{2}\right)=d^{n}-(d-1)^{k-1} d^{n-k+1}$. Il s'ensuit

$$
(n+1) ! \int_{Q_{\mathcal{A}}} \vartheta_{\mathcal{A}, \tau_{\alpha v}}(u) d u=(n+1) !\left(I_{1}+I_{2}+I_{3}\right)=d^{n}(\theta+E(\mathcal{A}, \alpha, v))
$$

avec

$$
\begin{aligned}
\theta & =\left(1-\frac{1}{d}\right)^{k-1}\left(\frac{d-f}{d}\left(k q_{0}+q_{k}+\cdots+q_{n}\right)+\frac{f}{d}\left((k+1) q_{0}+q_{k+1}+\cdots+q_{n}\right)\right) \log (2) \\
& =\left(1-\frac{1}{d}\right)^{k-1}\left(\left(k q_{0}+q_{k}+\cdots+q_{n}\right)+\frac{f}{d}\left(q_{0}-q_{k}\right)\right) \log (2)
\end{aligned}
$$

et

$0 \leq E(\mathcal{A}, \alpha, v)=\frac{(n+1) !}{d^{n}} I_{3} \leq(n+1)\left(1-\left(1-\frac{1}{d}\right)^{k-1}\right) q_{0} \log (2) \leq \frac{(n+1)(k-1)}{d} q_{0} \log (2)$.

On déduit alors du théorème $\$ .1$

$$
0 \leq \theta-\frac{\widehat{h}(X)}{\operatorname{deg}(X)} \leq \frac{(n+1)(k-1)}{d} q_{0} \log (2) .
$$


Maintenant on fixe les paramètres : d'abord on prend $\ell:=\left\lceil\log (2) \varepsilon_{1}^{-1}\right\rceil+1$, et pour $0 \leq i \leq n$ on pose $q_{i}:=\frac{1}{\ell}\left\lfloor\frac{\ell \cdot \mu_{i+1}}{\log (2)}\right\rfloor$ de sorte que $\widehat{\mu}_{i+1}(X)=q_{i} \log (2)$ satisfait

$$
0 \leq \mu_{i+1}-q_{i} \log (2)<\varepsilon_{1}
$$

comme voulu. On vérifie

$$
\left(q_{0}+\cdots+q_{n}\right) \log (2) \leq \mu_{1}+\cdots+\mu_{n+1} \leq \nu \leq(n+1) \mu_{1}-\varepsilon_{1} \leq(n+1) q_{0} \log (2)
$$

et on fixe $1 \leq k \leq n$ tel que

$$
\left(k q_{0}+q_{k}+\cdots+q_{n}\right) \log (2) \leq \nu \leq\left((k+1) q_{0}+q_{k+1}+\cdots+q_{n}\right) \log (2) .
$$

Soit $\lambda \in[0,1]$ tel que

$$
\nu=(1-\lambda)\left(k q_{0}+q_{k}+\cdots+q_{n}\right) \log (2)+\lambda\left((k+1) q_{0}+q_{k+1}+\cdots+q_{n}\right) \log (2) .
$$

On prend alors $1 \leq f \leq d-1$ tel que

$$
|\lambda-f / d| \leq 1 / d
$$

et on considère le $\theta=\theta(d)$ associé à ces paramètres. On vérifie facilement

$$
\begin{aligned}
|\nu-\theta| & \leq\left|\lambda-\frac{f}{d}\right|\left(q_{0}-q_{k}\right) \log (2)+\left(1-\left(1-\frac{1}{d}\right)^{k-1}\right) \cdot\left((k+1) q_{0}+q_{k+1}+\cdots+q_{n}\right) \log (2) \\
& \leq \frac{\mu_{1}}{d}+\frac{(k-1)(n+1) \mu_{1}}{d}=\frac{n^{2} \mu_{1}}{d} .
\end{aligned}
$$

Finalement on obtient le résultat cherché en sommant avec l'inégalité (VI.14)

$$
\left|\frac{\widehat{h}(X)}{\operatorname{deg}(X)}-\nu\right| \leq \frac{(n+1)(k-1)}{d} \mu_{1}+\frac{(n+1)(k-1)+1}{d} \mu_{1} \leq \frac{2 n^{2}}{d} \mu_{1} .
$$

En prenant, grâce au postulat de Bertrand, $d$ un premier entre $2 n^{2} \mu_{1} \varepsilon_{2}^{-1}$ et $4 n^{2} \mu_{1} \varepsilon_{2}^{-1}$ on arrive au résultat annoncé.

Ce résultat montre que déjà dans le cadre torique, toute configuration possible des minimums $\widehat{\mu}_{1}(X), \ldots, \widehat{\mu}_{n+1}(X)$ se réalise, et l'encadrement (11) est optimal en toute dimension, lorsque le degré de $X$ et celui du corps de définition sont assez grands. Toutefois, notre exemple présente une codimension minimale $N-n=2 n+1$ de l'ordre de la dimension de la variété produite. La question se pose donc de savoir ce qu'il en est pour les variétés de petite codimension. Dans le cas de codimension 1 on a le résultat suivant qui laisse ouverte la possibilité de raffinements de (11) en petit codimension.

Proposition VI.3. - Soit $X \subset \mathbf{P}^{N}$ une hypersurface torique d'équation homogène minimale $f_{X}=x^{b}-\lambda x^{c} \in \overline{\mathbf{Q}}\left[x_{0}, \ldots, x_{N}\right]$, alors

$$
\widehat{\mu}^{\mathrm{ess}}(X)=\frac{h(1: \lambda)}{\operatorname{deg}\left(f_{X}\right)}=\frac{\widehat{h}(X)}{\operatorname{deg}(X)}, \quad \widehat{\mu}_{2}(X)=\cdots=\widehat{\mu}_{N}(X)=0 .
$$

Démonstration. - Les contributions des places archimédiennes à la hauteur normalisée de $X$ sont égales aux mesures de Mahler des conjuguées de $f_{X}$ et donc $\widehat{h}(X)=h(1: \lambda)$, voir [PS04, suite de l'exemple III.7. Par la proposition $\llbracket$.1 on a $\lambda=\alpha^{b-c}$ pour tout point $\alpha \in X^{\circ}$. En choisissant $\alpha$ de hauteur normalisée aussi proche du minimum essentiel $\widehat{\mu}^{\text {ess }}(X)$ que l'on veut, on a

$$
\frac{\widehat{h}(X)}{\operatorname{deg}(X)}=\frac{h\left(1: \alpha^{b-c}\right)}{\operatorname{deg}\left(f_{X}\right)}=\frac{h\left(\alpha^{b}: \alpha^{c}\right)}{\operatorname{deg}\left(f_{X}\right)} \leq \widehat{h}(\alpha) \leq \widehat{\mu}^{\operatorname{ess}}(X)+\varepsilon
$$


pour tout $\varepsilon>0$. Avec la formule (11) de l'introduction on obtient $\widehat{\mu}_{1}(X)+\cdots+\widehat{\mu}_{n+1}(X) \leq$ $\widehat{h}(X) / \operatorname{deg}(X) \leq \widehat{\mu}_{1}(X)$, ce qui entraîne l'énoncé.

Il serait très intéressant d'expliciter les minimums successifs d'une variété torique quelconque, en généralisant à la fois le lemme VI.2 et la proposition VI.3. Grâce au lemme VI.1 on sait qu'il suffit de le faire pour le minimum essentiel.

Une question liée est celle de construire explicitement des points de $X_{\mathcal{A}, \alpha}^{\circ}$ de hauteur comparable au minimum essentiel. De plus, on peut se demander s'il existe un point dans la variété réalisant ce minimum essentiel, c'est-à-dire de savoir s'il existe un point de hauteur minimale. Dans le même ordre d'idée, existe-t-il un point de $X_{\mathcal{A}, \alpha}$ dont la hauteur normalisée soit égale au quotient $\widehat{h}\left(X_{\mathcal{A}, \alpha}\right) / \operatorname{deg}\left(X_{\mathcal{A}, \alpha}\right)$ ?

Pour conclure ce paragraphe, explicitons l'encadrement pour le quotient hauteur-sur-degré qui découle de la formule pour la hauteur d'une variété torique:

Proposition VI.4. - Soient $\mathcal{A} \in\left(\mathbf{Z}^{n}\right)^{N+1}$ et $\alpha \in\left(\overline{\mathbf{Q}}^{\times}\right)^{N+1}$, alors

$$
\widehat{h}(\alpha)-n \widehat{h}\left(\alpha_{j}^{-1}: a_{j} \in \mathrm{F}_{0}\left(Q_{\mathcal{A}}\right)\right) \leq \frac{\widehat{h}\left(X_{\mathcal{A}, \alpha}\right)}{\operatorname{deg}\left(X_{\mathcal{A}, \alpha}\right)} \leq(n+1) \widehat{h}(\alpha)
$$

où $a_{j}$ parcours l'ensemble $\mathrm{F}_{0}\left(Q_{\mathcal{A}}\right)$ des sommets de $Q_{\mathcal{A}}$.

Démonstration. - Soit $K$ le corps de définition de $\alpha$ et $v \in M_{K}$. On a $\max \left(\vartheta_{\mathcal{A}, \tau_{\alpha} v}\right)=$ $\log \max \left\{\left|\alpha_{0}\right|_{v}, \ldots,\left|\alpha_{N}\right|_{v}\right\}=: \log \left(\|\alpha\|_{v}\right)$, ce qui entraîne

$$
\int_{Q_{\mathcal{A}}} \vartheta_{\mathcal{A}, \tau_{\alpha v}}(u) d u \leq \operatorname{Vol}_{n}\left(Q_{\mathcal{A}}\right) \log \left(\|\alpha\|_{v}\right)=\frac{1}{n !} \operatorname{deg}\left(X_{\mathcal{A}, \alpha}\right) \log \left(\|\alpha\|_{v}\right)
$$

et donc $\widehat{h}\left(X_{\mathcal{A}, \alpha}\right) \leq(n+1) \operatorname{deg}\left(X_{\mathcal{A}, \alpha}\right) \widehat{h}(\alpha)$, ce qui établit la majoration. Pour la minoration, soit $v \in M_{K}$ et posons

$$
m_{v}:=\min _{u \in Q_{\mathcal{A}}}\left(\vartheta_{\mathcal{A}, \tau_{\alpha v}}(u)\right)=\min \left\{\max \left\{\log \left|\alpha_{i}\right|_{v}: 0 \leq i \leq N, a_{i}=a\right\}: a \in \mathrm{F}_{0}\left(Q_{\mathcal{A}}\right)\right\}
$$

et considérons le polytope $Q_{v}:=\operatorname{Conv}\left(\left(a_{i}, \log \left|\alpha_{i}\right|_{v}\right),\left(a_{i}, m_{v}\right): i=0, \ldots, N\right) \subset \mathbf{R}^{N+1}$. Soit $0 \leq \ell \leq N$ tel que $\log \left|\alpha_{\ell}\right|_{v}$ soit maximal, alors $Q_{v} \supset \operatorname{Conv}\left(\left(a_{\ell}, \log \left|\alpha_{\ell}\right|_{v}\right), Q_{\mathcal{A}} \times\left\{m_{v}\right\}\right)$ et donc $\operatorname{Vol}_{n+1}\left(Q_{v}\right) \geq \frac{1}{n+1}\left(\log \|\alpha\|_{v}-m_{v}\right) \operatorname{Vol}_{n}\left(Q_{\mathcal{A}}\right)$. On en déduit

$$
\int_{Q_{\mathcal{A}}} \vartheta_{\mathcal{A}, \tau_{\alpha v}}(u) d u=\operatorname{Vol}_{n+1}\left(Q_{v}\right)+m_{v} \operatorname{Vol}_{n}\left(Q_{\mathcal{A}}\right) \geq\left(\frac{1}{n+1} \log \left(\|\alpha\|_{v}\right)+\frac{n}{n+1} m_{v}\right) \operatorname{Vol}_{n}\left(Q_{\mathcal{A}}\right) \text {, }
$$

d'où $\widehat{h}\left(X_{\mathcal{A}, \alpha}\right) \geq \operatorname{deg}\left(X_{\mathcal{A}, \alpha}\right)\left(\widehat{h}(\alpha)-n \widehat{h}\left(\alpha_{j}^{-1}: a_{j} \in \mathrm{F}_{0}\left(Q_{\mathcal{A}}\right)\right)\right) \operatorname{car} \sum_{v \in M_{K}} \frac{\left[K_{v}: \mathbf{Q}_{v}\right]}{[K: \mathbf{Q}]}\left(-m_{v}\right) \leq$ $\widehat{h}\left(\alpha_{j}^{-1}: a_{j} \in \mathrm{F}_{0}\left(Q_{\mathcal{A}}\right)\right)$.

Notons qu'en remplaçant le point $\alpha$ par un point de $X_{\mathcal{A}, \alpha}^{\circ}$ de hauteur aussi proche que l'on veut du minimum essentiel, on retrouve simplement la majoration de (1); par contre on obtient une minoration différente.

\section{Poids de Chow et hauteur des diviseurs monomiaux}

Dans ce paragraphe on considère l'intersection d'une variété torique avec un diviseur monomial de $\mathbf{P}^{N}$. On montrera comment dans cette situation, le théorème de Bézout pour les poids de Chow (Théorème 0.2 de l'introduction et Théorème IV.2) s'explicite comme la décomposition polyédrale d'un certain volume, et dans cette situation peut se démontrer de façon indépendante. En combinant ceci avec la formule pour la hauteur d'une variété torique 
(théorème $\mathbf{\nabla . 1}$ ), on obtient un théorème de Bézout arithmétique pour la hauteur normalisée du cycle intersection d'une variété torique avec un diviseur monomial.

Soient $\mathcal{A} \in\left(\mathbf{Z}^{n}\right)^{N+1}$ tel que $L_{\mathcal{A}}=\mathbf{Z}^{n}$ et $b \in \mathbf{Z}^{N+1}$, notons $X_{\mathcal{A}} \subset \mathbf{P}^{N}$ et $\operatorname{div}\left(x^{b}\right) \in \operatorname{Div}\left(\mathbf{P}^{N}\right)$ la variété torique et le diviseur monomial associés. On s'intéressera au cycle intersection découpé sur $X_{\mathcal{A}}$ par le monôme $x^{b}$; le lemme ci-dessous explicite ce cycle.

Pour chaque hyperface $F \in F_{n-1}\left(Q_{\mathcal{A}}\right)$ on considère la variété $X_{\mathcal{A}, F} \subset \mathbf{P}^{N}$, adhérence de Zariski de l'orbite associée $X_{\mathcal{A}, F}^{\circ}$. On considère aussi l'hyperplan d'appui $H_{F} \subset \mathbf{R}^{n}$ de cette face et $H_{F}^{\mathbf{Z}}:=H_{F} \cap \mathbf{Z}^{n}$; fixons également un point quelconque $a_{F} \in F$. Notons $L_{\mathcal{A}, F}$ le $\mathbf{Z}$ module engendré par les différences des éléments de $\mathcal{A} \cap F$, qui est un sous-réseau de $H_{F}^{\mathbf{Z}}-a_{F}$ d'indice

$$
i(\mathcal{A} ; F):=\left[H_{F}^{\mathbf{Z}}-a_{F}: L_{\mathcal{A}, F}\right] .
$$

Rappelons que $v_{F} \in \mathbf{Z}^{n}$ désigne le plus petit vecteur entier, orthogonal à $H_{F}$ et dirigé vers l'intérieur de $Q_{\mathcal{A}}$. On pose $M_{\mathcal{A}}(b)=b_{0} a_{0}+\cdots+b_{N} a_{N} \in \mathbf{Z}^{n}$ et $D:=\operatorname{deg}\left(x^{b}\right)=\sum_{j=0}^{N} b_{j}$.

Lemme VII.1. - Avec les notations ci-dessus, on a

$$
X_{\mathcal{A}} \cdot \operatorname{div}\left(x^{b}\right)=\sum_{F \in \mathrm{F}_{n-1}\left(Q_{\mathcal{A}}\right)}\left\langle M_{\mathcal{A}}(b)-D a_{F}, v_{F}\right\rangle i(\mathcal{A} ; F)\left[X_{\mathcal{A}, F}\right] \in Z_{n-1}\left(\mathbf{P}^{N}\right)
$$

où $\langle\cdot, \cdot\rangle$ désigne le produit scalaire ordinaire de $\mathbf{R}^{n}$ et $\left[X_{\mathcal{A}, F}\right]$ le cycle défini par $X_{\mathcal{A}, F}$.

En particulier, un cycle $\left[X_{\mathcal{A}, F}\right]$ intervient comme composante de $X_{\mathcal{A}} \cdot \operatorname{div}\left(x^{b}\right)$ si et seulement si $M_{\mathcal{A}}(b)$ n'appartient pas à l'hyperplan $H_{F}+(D-1) a_{F}$.

Démonstration. - Grâce à la décomposition en orbites (I.3) on vérifie que le cycle considéré est supporté par la réunion des orbites de codimension 1, celles-ci correspondant aux hyperfaces de $Q_{\mathcal{A}}$, soit

$$
\bigcup_{F \in \mathrm{F}_{n-1}\left(Q_{\mathcal{A}}\right)} X_{\mathcal{A}, F}
$$

Explicitons les multiplicités correspondantes. Par linéarité on peut se ramener sans perte de généralité au cas où $b=e_{i}$ est un des vecteurs de la base standard de $\mathbf{R}^{N+1}$ pour un certain $0 \leq i \leq N$, c'est-à-dire $x^{b}=x_{i}$. Fixons une hyperface $F$ et soit $a_{j} \in F$ un sommet quelconque. Considérons le cône dual de l'angle de $Q_{\mathcal{A}}$ en $a_{j}$ :

$$
\sigma:=\left\{u \in \mathbf{R}^{n}:\left\langle u, a_{k}-a_{j}\right\rangle \geq 0 \text { pour } k=0, \ldots, N\right\} \subset \mathbf{R}^{n},
$$

et soit $U_{\sigma}$ la variété torique affine correspondante, définie par

$$
U_{\sigma}:=\operatorname{Spec}\left(\overline{\mathbf{Q}}\left[S_{\sigma}\right]\right)
$$

où $S_{\sigma}:=\sigma^{\vee} \cap \mathbf{Z}^{n}$ est le semi-groupe des points entiers dans l'angle de $Q_{\mathcal{A}}$ en $a_{j}$ [Ful93 $\left.\S 1.3\right]$. Considérons encore l'application naturelle $N: U_{\sigma} \rightarrow\left(X_{\mathcal{A}}\right)_{x_{j}} \subset\left(\mathbf{P}^{N}\right)_{x_{j}}$ donnée par l'inclusion d'algèbres

$$
\overline{\mathbf{Q}}\left[\left(X_{\mathcal{A}}\right)_{x_{j}}\right]=\overline{\mathbf{Q}}\left[s^{a_{0}-a_{j}}, \ldots, s^{a_{N}-a_{j}}\right] \hookrightarrow \overline{\mathbf{Q}}\left[S_{\sigma}\right]=\overline{\mathbf{Q}}\left[U_{\sigma}\right] .
$$

La variété $U_{\sigma}$ est normale car $S_{\sigma}$ est un semi-groupe saturé, et de ce fait $N$ est le morphisme de normalisation de la carte affine $\left(X_{\mathcal{A}}\right)_{x_{j}}$ Stu96, Cor. 13.6]. 
Soit $\rho$ l'arête du cône $\sigma$ duale de la face $F$, notons $V(\rho)$ la clôture dans $U_{\sigma}$ de l'orbite correspondante [Ful93, §3.1]. On a un diagramme commutatif

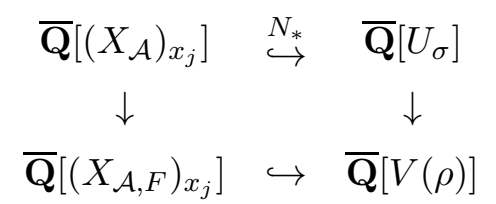

qui implique $N^{-1}\left(X_{\mathcal{A}, F}\right)=V(\rho)$ et $\operatorname{deg}\left(\left.N\right|_{V(\rho)}\right)=\left[H_{F}^{\mathbf{Z}}: L_{\mathcal{A}, F}\right]=i(\mathcal{A} ; F)$. Le monôme $\chi:=N^{*}\left(x_{i}\right)=s^{a_{i}-a_{j}} \in \overline{\mathbf{Q}}\left[s_{1}^{ \pm 1}, \ldots, s_{n}^{ \pm 1}\right]$ définit une fonction rationnelle $U_{\sigma} \rightarrow \overline{\mathbf{Q}}$. On a $\operatorname{ord}_{X_{\mathcal{A}, F}}\left(x_{i}\right)=\operatorname{deg}\left(\left.N\right|_{V(\rho)}\right) \operatorname{ord}_{V(\rho)}(\chi)$ puisque $U_{\sigma}$ est normal Ful84, Exerc. 1.2.3.]. On en déduit

$$
\begin{aligned}
\operatorname{long}_{\overline{\mathbf{Q}}\left[\left(X_{\mathcal{A}, F}\right)_{x_{j}}\right]}\left(\overline{\mathbf{Q}}\left[\left(X_{\mathcal{A}}\right)_{x_{j}}\right] /\left(x_{i}\right)\right) & =\operatorname{ord}_{X_{\mathcal{A}, F}}\left(x_{i}\right) \\
& =\operatorname{deg}\left(\left.N\right|_{V(\rho)}\right) \operatorname{ord}_{V(\rho)}(\chi)=i(\mathcal{A} ; F) \operatorname{ord}_{V(\rho)}(\chi) .
\end{aligned}
$$

Finalement, le lemme de Ful93 $\S 3.3$, p. 61] entraîne $\operatorname{ord}_{V(\rho)}(\chi)=\left\langle a_{i}-a_{j}, v_{F}\right\rangle$ car $v_{F}$ est le générateur du semi-groupe $\rho \cap \mathbf{Z}^{n} \cong \mathbf{N}$, d'où

$$
m\left(X_{\mathcal{A}} \cdot \operatorname{div}\left(x_{i}\right) ; X_{\mathcal{A}, F}\right)=\operatorname{long}_{\overline{\mathbf{Q}}\left[\left(X_{\mathcal{A}, F}\right)_{x_{j}}\right]}\left(\overline{\mathbf{Q}}\left[\left(X_{\mathcal{A}}\right)_{x_{j}}\right] /\left(x_{i}\right)\right)=\left\langle a_{i}-a_{j}, v_{F}\right\rangle i(\mathcal{A} ; F) .
$$

Le théorème de Bézout géométrique

$$
\operatorname{deg}\left(X_{\mathcal{A}} \cdot \operatorname{div}\left(x^{b}\right)\right)=D \operatorname{deg}\left(X_{\mathcal{A}}\right)
$$

s'interprête en termes de décomposition du volume du polytope $Q_{\mathcal{A}}$ : on a $\operatorname{deg}\left(X_{\mathcal{A}}\right)=$ $n ! \operatorname{Vol}_{n}\left(Q_{\mathcal{A}}\right)$ et

$$
\begin{aligned}
\operatorname{deg}\left(X_{\mathcal{A}, F}\right) & =(n-1) ! \mu_{\mathcal{A}(F)}(F) \\
& =\frac{(n-1) !}{\operatorname{Vol}_{n-1}\left(\left(H_{F}-a_{F}\right) / L_{\mathcal{A}, F}\right)} \operatorname{Vol}_{n-1}(F)=\frac{(n-1) !}{i(\mathcal{A} ; F)\left\|v_{F}\right\|_{2}} \operatorname{Vol}_{n-1}(F),
\end{aligned}
$$

à cause de la normalisation de la forme volume $\mu_{\mathcal{A}(F)}$ sur $H_{F}$ et du fait que

$$
\operatorname{Vol}_{n-1}\left(H_{F} / L_{\mathcal{A}, F}\right)=i(\mathcal{A} ; F) \cdot \operatorname{Vol}_{n-1}\left(H_{F} / H_{F}^{\mathbf{Z}}\right)=i(\mathcal{A} ; F) \cdot\left\|v_{F}\right\|_{2},
$$

conséquence de la formule de Brill-Gordan. On a encore

$$
\left\langle M_{\mathcal{A}}(b)-D a_{F}, v_{F}\right\rangle=\varepsilon(b, F)\left\|v_{F}\right\|_{2} \operatorname{dist}\left(M_{\mathcal{A}}(b), H_{F}+(D-1) a_{F}\right),
$$

où $\varepsilon(b, F)=+1$ si $M_{\mathcal{A}}(b)$ et $v_{F}$ sont d'un même côté de l'hyperplan $H_{F}+(D-1) a_{F}$ et $\varepsilon(b, F)=-1$ sinon. Combiné avec le lemme VII.1 cela donne

$$
\begin{aligned}
\operatorname{deg}\left(X_{\mathcal{A}} \cdot \operatorname{div}\left(x^{b}\right)\right) & =\sum_{F \in \mathrm{F}_{n-1}\left(Q_{\mathcal{A}}\right)}\left\langle M_{\mathcal{A}}(b)-D a_{F}, v_{F}\right\rangle i(\mathcal{A}, F) \operatorname{deg}\left(X_{\mathcal{A}, F}\right) \\
& =\sum_{F \in \mathrm{F}_{n-1}\left(Q_{\mathcal{A}}\right)} \varepsilon(b, F)(n-1) ! \operatorname{Vol}_{n-1}(F) \operatorname{dist}\left(M_{\mathcal{A}}(b), H_{F}+(D-1) a_{F}\right) \\
& =\sum_{F \in \mathrm{F}_{n-1}\left(Q_{\mathcal{A}}\right)} n ! \varepsilon(b, F) \operatorname{Vol}_{n}\left(\operatorname{Conv}\left(F+(D-1) a_{F}, M_{\mathcal{A}}(b)\right)\right) .
\end{aligned}
$$

L'identité (VII.17) ci-dessus (multipliée par $n !^{-1}$ ) se traduit ainsi en la décomposition de volume

(VII.18)

$$
\sum_{F \in \mathrm{F}_{n-1}\left(Q_{\mathcal{A}}\right)} \varepsilon(b, F) \operatorname{Vol}_{n}\left(\operatorname{Conv}\left(F+(D-1) a_{F}, M_{\mathcal{A}}(b)\right)\right)=D \operatorname{Vol}_{n}\left(Q_{\mathcal{A}}\right)
$$


La figure suivante illustre cette décomposition, pour $\mathcal{A}=((1,1),(1,0),(3,1),(2,2),(0,2)) \in$ $\left(\mathbf{Z}^{2}\right)^{6}$ et $b=(1,0,0,0,0)\left(\operatorname{donc} M_{\mathcal{A}}(b)=(1,1)=a_{0}\right.$ et $\left.D=1\right)$ :

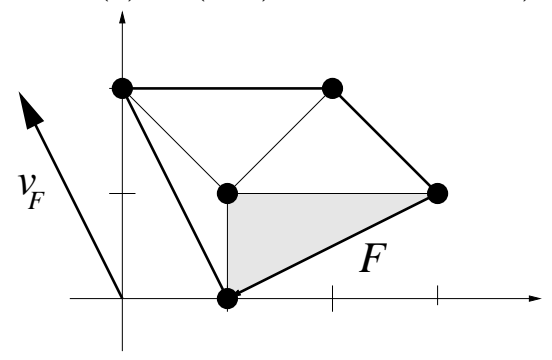

Soit $\tau=\left(\tau_{0}, \ldots, \tau_{N}\right) \in \mathbf{Z}^{N+1}$. Pour le cas $\tau \in\left(\mathbf{N}^{\times}\right)^{N+1}$, le théorème de Bézout pour les poids de Chow (théorème IV.2) s'écrit dans les notations du $\S$ IV

$\left(\right.$ VII.19) $e_{\tau}\left(X_{\mathcal{A}} \cdot \operatorname{div}\left(x^{b}\right)\right)=D e_{\tau}\left(X_{\mathcal{A}}\right)-\sum_{Y \in \operatorname{Irr}\left(\operatorname{init}_{\tau}\left(X_{\mathcal{A}}\right)\right)} m\left(\left(X_{\mathcal{A}}\right)_{\tau} \cdot \operatorname{div}\left(\lambda_{\tau}^{*}\left(x^{b}\right)\right) ; \iota(Y)\right) \cdot \operatorname{deg}(Y)$.

On va expliciter les termes intervenant dans cet enoncé. Soit

$$
Q_{\mathcal{A}, \tau}=\operatorname{Conv}\left(\left(a_{0}, \tau_{0}\right), \ldots,\left(a_{N}, \tau_{N}\right)\right) \subset \mathbf{R}^{n+1}
$$

le polytope associé au couple $(\mathcal{A}, \tau)$, dont la toiture $E_{\mathcal{A}, \tau}$ (c'est-à-dire l'enveloppe supérieure) s'envoie bijectivement sur $Q_{\mathcal{A}}$ par la projection $\mathbf{R}^{n} \times \mathbf{R} \rightarrow \mathbf{R}^{n}$. On appelle pan de la toiture toute face de dimension $n$ de $E_{\mathcal{A}, \tau}$. De même, on appelle mur tout polytope de la forme $\operatorname{Conv}\left(Q_{\mathcal{A}(F)}, F \times\{0\}\right)$ pour une hyperface $F$ de $Q_{\mathcal{A}}$; dans le cas $\tau \geq 0$ ceci est un des murs de la maison $\mathcal{M}_{\mathcal{A}, \tau}:=\operatorname{Conv}\left(Q_{\mathcal{A}, \tau} \cup\left(Q_{\mathcal{A}} \times\{0\}\right)\right)$, c'est-à-dire une face de dimension $n$ se projetant sur une face de dimension $n-1$ de $Q_{\mathcal{A}}$.

La déformation torique $\left(X_{\mathcal{A}}\right)_{\tau}$ est l'adhérence de Zariski de l'application

$$
\mathbf{G}_{m} \times \mathbf{G}_{m}^{n} \rightarrow \mathbf{P}^{1} \times \mathbf{P}^{N}, \quad(t, s) \rightarrow\left((1: t),\left(t^{\tau_{0}} s^{a_{0}}: \cdots: t^{\tau_{N}} s^{a_{N}}\right)\right)
$$

C'est donc la variété torique bi-projective associée aux vecteurs $(0,1) \in\left(\mathbf{Z}^{1}\right)^{2}$ et $(\tau, \mathcal{A})=$ $\left(\left(\tau_{0}, a_{0}\right), \ldots,\left(\tau_{N}, a_{N}\right)\right) \in\left(\mathbf{Z} \times \mathbf{Z}^{n}\right)^{N+1}$, voir $\S$ 【e couple de polytopes associé est donc $\mathbf{0} \times[0,1]$ et $Q_{\mathcal{A}, \tau} ;$ comme conséquence de la décomposition en orbites décrite au $\S$ प on vérifie que les points de $\left(X_{\mathcal{A}}\right)_{\tau}$ contenus dans l'hyperplan $\{(0: 1)\} \times \mathbf{P}^{N}$ correspondent nécessairement aux couples de faces de la forme $(\{(\mathbf{0}, 1)\}, F)$ avec $F \in \mathrm{F}_{n}\left(E_{\mathcal{A}, \tau}\right)$. On voit ainsi qu'il y a bijection entre les pans de $E_{\mathcal{A}, \tau}$ et les orbites de $X_{\mathcal{A}, \tau}$ contenues dans l'hyperplan $\{(0: 1)\} \times \mathbf{P}^{N}$, en particulier le support $\operatorname{de} X_{\mathcal{A}, \tau} \cdot\left(\{(0: 1)\} \times \mathbf{P}^{N}\right)$ est contenu dans

$$
\bigcup_{P \in \mathrm{F}_{n}\left(E_{\mathcal{A}, \tau}\right)} X_{\mathcal{A}, \tau, P}
$$

L'identification $\iota: \mathbf{P}^{N} \rightarrow\{(0: 1)\} \times \mathbf{P}^{N} \subset \mathbf{P}^{1} \times \mathbf{P}^{N}$ met en correspondance le cycle $X_{\mathcal{A}, \tau} \cdot(\{(0$ : 1) $\} \times \mathbf{P}^{N}$ ) et la variété initiale $\operatorname{init}_{\tau}\left(X_{\mathcal{A}}\right)$, on en déduit qu'il y a bijection entre les composantes

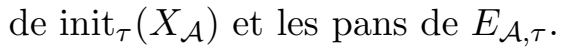

Pour chaque pan $P$ on considère son hyperplan d'appui $H_{P} \subset \mathbf{R}^{n+1}$, posons $L_{\mathcal{A}, \tau, P} \subset \mathbf{Z}^{n+1}$ le $\mathbf{Z}$-module engendré par les différences des éléments de $(\tau, \mathcal{A}) \cap P$. Modulo une translation, ce dernier est un sous-réseau de $H_{P}^{\mathbf{Z}}:=H_{P} \cap \mathbf{Z}^{n+1}$ d'indice

$$
i(\mathcal{A}, \tau ; P):=\left[H_{P}^{\mathbf{Z}}: L_{\mathcal{A}, \tau, P}\right] \text {. }
$$

Avec ces notations, d'après [Stu94, formule (27), page 222] (voir aussi [KSZ92, Thm. 5.3.]), on a

$$
X_{\mathcal{A}, \tau} \cdot\left(\{(0: 1)\} \times \mathbf{P}^{N}\right)=\iota\left(\operatorname{init}_{\tau}\left(X_{\mathcal{A}}\right)\right)=\sum_{P \in \mathrm{F}_{n}\left(E_{\mathcal{A}, \tau}\right)} i(\mathcal{A}, \tau ; P)\left[X_{\mathcal{A}, \tau, P}\right]
$$


Pour chaque pan $P$ on note $\left(v_{P}, w_{P}\right) \in \mathbf{Z}^{n} \times \mathbf{Z}$ le plus petit vecteur entier orthogonal au plan d'appui $H_{P} \subset \mathbf{R}^{n+1}$ tel que $w_{P}<0$. On note aussi $\left(a_{P}, \tau_{P}\right)$ un point quelconque de $P$.

Le vecteur $\tau$ induit une décomposition polyédrale cohérente $\mathrm{DPC}_{\tau}\left(Q_{\mathcal{A}}\right)$ du polytope de base $Q_{\mathcal{A}}$. Les faces $S$ de dimension $n$ de cette décomposition sont en correspondance avec les pans de la toiture; pour $S \in \operatorname{DPC}_{\tau}\left(Q_{\mathcal{A}}\right)$ on écrit $\operatorname{Pan}(S) \in \mathrm{F}_{n}\left(E_{\mathcal{A}, \tau}\right)$ pour le pan correspondant.

Lemme VII.2. - Soient $\tau \in \mathbf{Z}^{N+1}$ et $P \in \mathrm{F}_{n}\left(E_{\mathcal{A}, \tau}\right)$ un pan de la toiture de $Q_{\mathcal{A}, \tau}$ alors,

$$
m\left(X_{\mathcal{A}, \tau} \cdot \operatorname{div}\left(\lambda_{\tau}^{*}\left(x^{b}\right)\right) ; X_{\mathcal{A}, \tau, P}\right)=\left(\left\langle M_{\mathcal{A}}(b)-D a_{P}, v_{P}\right\rangle-D \tau_{P} w_{P}\right) i(\mathcal{A}, \tau ; P),
$$

avec $\lambda_{\tau}^{*}\left(x^{b}\right)=\left(t^{\tau_{0}} x_{0}, \ldots, t^{\tau_{N}} x_{N}\right)^{b}=t_{0}^{b_{0} \tau_{0}+\cdots+b_{N} \tau_{N}} x^{b}$.

Démonstration. - Cette démonstration étant tout à fait analogue à celle du lemme VII.1 on n'indiquera que les pas principaux.

Par linéarité on peut se ramener au cas où $b=e_{i}$ est un des vecteurs de la base standard de $\mathbf{R}^{N+1}$, donc $x^{b}=x_{i}$. Soit $\left(a_{j}, \tau_{j}\right)$ un sommet quelconque du pan $P$, on se place dans la carte affine $\mathbf{A}^{1} \times \mathbf{A}^{N} \subset \mathbf{P}^{1} \times \mathbf{P}^{N}$ correspondant à $t_{1} \neq 0$ et $x_{j} \neq 0$ (puisqu'on veut calculer une multiplicité le long d'une sous-variété de $Z\left(t_{0}\right)$ ). Dans cette carte, l'application monomiale s'écrit

$$
\mathbf{G}_{m} \times \mathbf{G}_{m}^{n} \rightarrow \mathbf{A}^{1} \times \mathbf{A}^{N}, \quad(t, s) \mapsto\left(t ; t^{\tau_{j}-\tau_{0}} s^{a_{0}-a_{j}}, \ldots, t^{\tau_{j}-\tau_{N}} s^{a_{N}-a_{j}}\right)
$$

et donc l'algèbre de cette carte affine de $X_{\mathcal{A}, \tau}$ est

$$
\overline{\mathbf{Q}}\left[\left(X_{\mathcal{A}, \tau}\right)_{t_{1}, x_{j}}\right]=\overline{\mathbf{Q}}\left[t, t^{\tau_{j}-\tau_{0}} s^{a_{0}-a_{j}}, \ldots, t^{\tau_{j}-\tau_{N}} s^{a_{N}-a_{j}}\right] .
$$

La normalisation de cette algèbre correspond au cône

$$
\sigma:=\left\{(u, v) \in \mathbf{R}^{n} \times \mathbf{R}: v \geq 0,\left\langle u, a_{k}-a_{j}\right\rangle+v\left(\tau_{j}-\tau_{k}\right) \geq 0, k=0, \ldots, N\right\} .
$$

Le reste de la démonstration suit les lignes de celle du lemme VII.1 en considérant la normalisation de $\left(X_{\mathcal{A}, \tau}\right)_{t_{1}, x_{j}}$ donnée par le semi-groupe des points entiers $S_{\sigma}:=\sigma^{\vee} \cap \mathbf{Z}^{n}$.

L'hyperplan $H_{P} \subset \mathbf{R}^{n+1}$ est un des plans d'appui du cône $\sigma^{\vee}$, car $P$ est un pan. Il définit donc une arête $\rho$ du cône dual $\sigma$, dont le semi-groupe $\rho \cap \mathbf{Z}^{n}$ est engendré par $\left(v_{P},-w_{P}\right)$. Comme $\lambda_{\tau}^{*}\left(x_{i}\right)=t^{\tau_{i}} x_{i}=t^{\tau_{j}} s^{a_{i}-a_{j}}$ sur la carte considérée, on en conclut

$$
\begin{aligned}
m\left(X_{\mathcal{A}, \tau} \cdot \operatorname{div}\left(\lambda_{\tau}^{*}\left(x_{i}\right)\right) ; X_{\mathcal{A}, \tau, P}\right) & =\left\langle\left(a_{i}, 0\right)-\left(a_{j},-\tau_{j}\right),\left(v_{P},-w_{P}\right)\right\rangle i(\mathcal{A}, \tau ; P) \\
& =\left(\left\langle a_{i}-a_{j}, v_{P}\right\rangle-\tau_{j} w_{P}\right) i(\mathcal{A}, \tau ; P)
\end{aligned}
$$

Proposition VII.3. - Soit $\mathcal{A} \subset\left(\mathbf{Z}^{n}\right)^{N+1}$ tel que $L_{\mathcal{A}}=\mathbf{Z}^{n}, b \in \mathbf{Z}^{N+1}$ et $\tau \in \mathbf{N}^{N+1}$, alors l'égalité (DII.19) correspond terme à terme à la suivante, multipliée par $(n+1)$ !,

$$
\begin{aligned}
D \operatorname{Vol}_{n+1}\left(\mathcal{M}_{\mathcal{A}, \tau}\right)= & \sum_{F \in \mathrm{F}_{n-1}\left(Q_{\mathcal{A}}\right)} \varepsilon(b, F) \operatorname{Vol}_{n+1}\left(\operatorname{Conv}\left(M(F)+(D-1)\left(a_{F}, 0\right),\left(M_{\mathcal{A}}(b), 0\right)\right)\right) \\
& +\sum_{P \in \mathrm{F}_{n}\left(E_{\mathcal{A}, \tau}\right)} \varepsilon(b, P) \operatorname{Vol}_{n+1}\left(\operatorname{Conv}\left(P+(D-1)\left(a_{P}, \tau_{P}\right),\left(M_{\mathcal{A}}(b), 0\right)\right)\right)
\end{aligned}
$$

où $\varepsilon(b, F)=+1$ (resp. $\varepsilon(b, P)=+1)$ si $M_{\mathcal{A}}(b)$ et $v_{F}\left(\right.$ resp. $\left(M_{\mathcal{A}}(b), 0\right)$ et $\left.\left(v_{P}, w_{P}\right)\right)$ sont d'un même côté de l'hyperplan $H_{F}+(D-1) a_{F}$ (resp. $\left.H_{P}+(D-1)\left(a_{P}, \tau_{P}\right)\right)$ et $\varepsilon(b, F)=-1$ (resp. $\varepsilon(b, P)=-1)$ sinon.

La figure suivante (pour $b=e_{i}, D=1$ et $M_{\mathcal{A}}(b)=a_{i}$ ) illustre cette décomposition: 


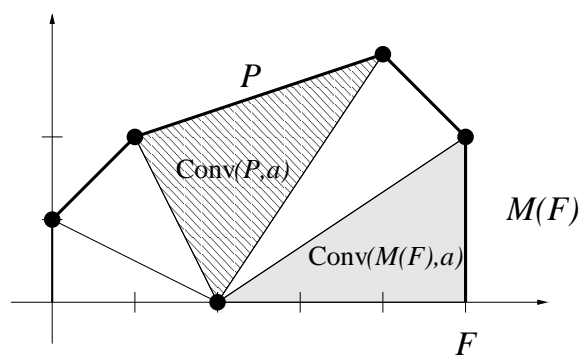

Démonstration. - Montrons comment (VII.19) se traduit en la décomposition de l'intégrale de la fonction $\vartheta_{\mathcal{A}, \tau}: Q_{\mathcal{A}} \rightarrow \mathbf{R}$ paramétrant la toiture $E_{\mathcal{A}, \tau}$ de la proposition VII.3. On a d'abord

$$
e_{\tau}\left(X_{\mathcal{A}}\right)=(n+1) ! \int_{Q_{\mathcal{A}}} \vartheta_{\mathcal{A}, \tau}(u) d u_{1} \cdots d u_{n}=(n+1) ! \operatorname{Vol}_{n+1}\left(\mathcal{M}_{\mathcal{A}, \tau}\right)
$$

puis, par le lemme VII.1 il vient

$$
e_{\tau}\left(X_{\mathcal{A}} \cdot \operatorname{div}\left(x^{b}\right)\right)=\sum_{F \in \mathrm{F}_{n-1}\left(Q_{\mathcal{A}}\right)}\left\langle M_{\mathcal{A}}(b)-D a_{F}, v_{F}\right\rangle i(\mathcal{A} ; F) e_{\tau}\left(X_{\mathcal{A}, F}\right)
$$

et pour chaque face $F$ de $Q_{\mathcal{A}}$

$$
e_{\tau}\left(X_{\mathcal{A}, F}\right)=\frac{n !}{i(\mathcal{A} ; F)\left\|v_{F}\right\|_{2}} \int_{F} \vartheta_{\mathcal{A}, \tau} d \mu_{n-1}=\frac{n !}{i(\mathcal{A} ; F)\left\|v_{F}\right\|_{2}} \operatorname{Vol}_{n}(M(F))
$$

où $M(F) \subset \mathbf{R}^{n+1}$ désigne le mur de la maison $\mathcal{M}_{\mathcal{A}, \tau}$ au-dessus de $F$. Ainsi

$$
\begin{array}{r}
\left\langle M_{\mathcal{A}}(b)-D a_{F}, v_{F}\right\rangle i(\mathcal{A} ; F) e_{\tau}\left(X_{\mathcal{A}, F}\right)=n ! \varepsilon(b, F) \operatorname{dist}\left(M_{\mathcal{A}}(b), F+(D-1) a_{F}\right) \operatorname{Vol}_{n}(M(F)) \\
=(n+1) ! \varepsilon(b, F) \operatorname{Vol}_{n+1}\left(\operatorname{Conv}\left(M(F)+(D-1)\left(a_{F}, 0\right),\left(M_{\mathcal{A}}(b), 0\right)\right)\right) .
\end{array}
$$

Finalement, soit $Y$ une composante irréductible de $\operatorname{init}_{\tau}\left(X_{\mathcal{A}}\right)$ puis $S \in \operatorname{DPC}_{\tau}\left(Q_{\mathcal{A}}\right)$ et $P:=\operatorname{Pan}(S) \in \mathrm{F}_{n}\left(E_{\mathcal{A}, \tau}\right)$ les faces correspondantes dans la subdivision et dans la toiture respectivement, on a

$$
\operatorname{deg}(Y)=\frac{n !}{i(\mathcal{A} ; S)} \operatorname{Vol}_{n}(S)=\frac{n !}{i(\mathcal{A}, \tau ; P)\left\|\left(v_{P}, w_{P}\right)\right\|_{2}} \operatorname{Vol}_{n}(P) .
$$

Le lemme VII.2 entraîne alors

$$
\begin{array}{r}
m\left(X_{\mathcal{A}, \tau} \cdot \operatorname{div}\left(\lambda_{\tau}^{*}\left(x^{b}\right)\right) ; \iota(Y)\right) \operatorname{deg}(Y)=\frac{n !}{\prod\left(v_{P}, w_{P}\right) \|_{2}}\left\langle\left(M_{\mathcal{A}}(b), 0\right)-D\left(a_{P}, \tau_{P}\right),\left(v_{P}, w_{P}\right)\right\rangle \operatorname{Vol}_{n}(P) \\
=(n+1) ! \varepsilon(b, P) \operatorname{Vol}_{n+1}\left(\operatorname{Conv}\left(P+(D-1)\left(a_{P}, \tau_{P}\right),\left(M_{\mathcal{A}}(b), 0\right)\right)\right) .
\end{array}
$$

En regroupant ces calculs on voit que l'identité (VII.19) (multipliée par $\frac{1}{(n+1) !}$ ) se traduit dans la décomposition cherchée.

Pour $S \in \operatorname{DPC}_{\tau}\left(Q_{\mathcal{A}}\right)$ on définit $\theta_{\tau, S}(b) \in \mathbf{R}$ l'unique réel tel que $\left(M_{\mathcal{A}}(b), \theta_{\tau, S}(b)\right) \in$ $H_{\mathrm{Pan}(S)}+(D-1)\left(a_{\operatorname{Pan}(S)}, \tau_{\operatorname{Pan}(S)}\right)$. Le lemme suivant explicite cette quantité.

Lemme VII.4. - Soit $S \in \mathrm{DPC}_{\tau}\left(Q_{\mathcal{A}}\right)$ et $a_{j_{0}}, \ldots, a_{j_{n}} \in S$ des vecteurs affinement indépendants. Soit $b \in \mathbf{Z}^{N+1}$ et $D:=\sum_{j=0}^{N} b_{j}$, alors

$$
\theta_{\tau, S}(b) \cdot \operatorname{det}\left[\begin{array}{ccc}
1 & \cdots & 1 \\
a_{j_{0}, 1} & \cdots & a_{j_{n}, 1} \\
\vdots & \ddots & \vdots \\
a_{j_{0}, n} & \cdots & a_{j_{n}, n}
\end{array}\right]=-\operatorname{det}\left[\begin{array}{cccc}
1 & \cdots & 1 & D \\
a_{j_{0}, 1} & \cdots & a_{j_{n}, 1} & M_{\mathcal{A}}(b)_{1} \\
\vdots & \ddots & \vdots & \vdots \\
a_{j_{0}, n} & \cdots & a_{j_{n}, n} & M_{\mathcal{A}}(b)_{n} \\
\tau_{j_{0}} & \cdots & \tau_{j_{n}} & 0
\end{array}\right] .
$$


En triangulant $S$ par des simplexes et en utilisant la relation entre déterminant et volume, on peut réécrire ceci sous la forme (on pose $P=\operatorname{Pan}(S)$ ) :

$$
\begin{aligned}
\theta_{\tau, S}(b) \operatorname{Vol}_{n}(S) & =(n+1) \varepsilon(b, P) \operatorname{Vol}_{n+1}\left(\operatorname{Conv}\left(P+(D-1)\left(a_{P}, \tau_{P}\right),\left(M_{\mathcal{A}}(b), 0\right)\right)\right) \\
& =m\left(X_{\mathcal{A}, \tau} \cdot \operatorname{div}\left(\lambda_{\tau}^{*}\left(x^{b}\right)\right) ; \iota(Y)\right) \operatorname{deg}(Y)
\end{aligned}
$$

qui s'interprète comme l'égalité des volumes montrés dans la figure suivante (lorsque $D=1$ et en posant $\left.a=M_{\mathcal{A}}(b)\right)$ :

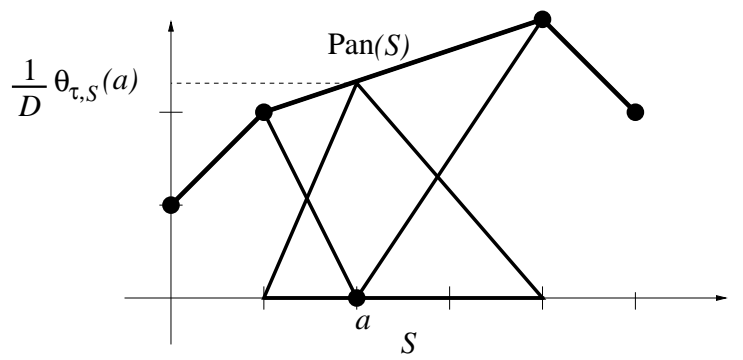

Démonstration. - C'est un calcul direct de l'intersection des espaces linéaires $H_{\mathrm{Pan}(S)}+(D-$ $1)\left(a_{\operatorname{Pan}(S)}, \tau_{\operatorname{Pan}(S)}\right)$ et $\left\{M_{\mathcal{A}}(b)\right\} \times \mathbf{R}:$ on a $\theta_{\tau, S}(b)=\sum_{i=0}^{n} v_{i} \tau_{j_{i}}$ où $v=\left(v_{0}, \ldots, v_{n}\right) \in \mathbf{R}^{n+1}$ est l'unique solution du système linéaire

$$
\sum_{i=0}^{n} v_{i}=D \quad, \quad \sum_{i=0}^{n} v_{i} a_{j_{i}}=M_{\mathcal{A}}(b) .
$$

On résout ce système par les formules de Cramer et on trouve ainsi

$\theta_{\tau, S}(b) \cdot \operatorname{det}\left[\begin{array}{ccc}1 & \cdots & 1 \\ a_{j_{0}, 1} & \cdots & a_{j_{n}, 1} \\ \vdots & \ddots & \vdots \\ a_{j_{0}, n} & \cdots & a_{j_{n}, n}\end{array}\right]=\sum_{i=0}^{n} \tau_{j_{i}} \operatorname{det}\left[\begin{array}{ccccccc}1 & \cdots & 1 & D & 1 & \ldots & 1 \\ a_{j_{0}, 1} & \cdots & a_{j_{i-1}, 1} & M_{\mathcal{A}}(b)_{1} & a_{j_{i+1}, 1} & \ldots & a_{j_{n}, 1} \\ \vdots & \ddots & \vdots & \vdots & \vdots & \ddots & \vdots \\ a_{j_{0}, n} & \cdots & a_{j_{i-1}, n} & M_{\mathcal{A}}(b)_{n} & a_{j_{i+1}, n} & \ldots & a_{j_{n}, n}\end{array}\right]$

qui est le développement du déterminant dans le membre droite de l'énoncé, par rapport à la dernière ligne.

Ceci permet d'écrire l'identité (VII.19) de la façon suivante:

Proposition VII.5. - Soit $\mathcal{A} \in\left(\mathbf{Z}^{n}\right)^{N+1}, b \in \mathbf{Z}^{N+1}$ et $\tau=\left(\tau_{0}, \ldots, \tau_{N}\right) \in \mathbf{R}^{N+1}$, posons $D:=\sum_{j=0}^{N} b_{j}$, alors

$$
e_{\tau}\left(X_{\mathcal{A}} \cdot \operatorname{div}\left(x^{b}\right)\right)=D e_{\tau}\left(X_{\mathcal{A}}\right)-n ! \sum_{S \in \operatorname{DPC}_{\tau}\left(Q_{\mathcal{A}}\right)} \theta_{\tau, S}(b) \operatorname{Vol}_{n}(S)
$$

où la seconde somme porte sur les faces $S$ de dimension $n$ de la décomposition polyédrale $\operatorname{DPC}_{\tau}\left(Q_{\mathcal{A}}\right)$.

Démonstration. - Le cas $\tau \in \mathbf{N}^{N+1}$ résulte de la proposition VII.3 et de (VII.20). Ceci s'étend successivement à $\tau \in \mathbf{Z}^{N+1}$ à cause de l'invariance de la formule par remplacement de $\tau$ par $\tau+c \cdot(1, \ldots, 1)$ avec $c \in \mathbf{N}$. Puis, la formule s'étend à $\tau \in \mathbf{Q}^{N+1}$ par homogénéité et finalement à $\tau \in \mathbf{R}^{N+1}$ par continuité.

Avec la notation (IV.9) on peut encore écrire cet énoncé sous la forme :

$$
w_{X_{\mathcal{A}, \tau}}\left(x^{b}\right)=-\sum_{S \in \operatorname{DPC}_{\tau}\left(Q_{\mathcal{A}}\right)} \theta_{\tau, S}(b) \cdot \frac{\operatorname{Vol}_{n}(S)}{\operatorname{Vol}_{n}\left(Q_{\mathcal{A}}\right)},
$$


dont on vérifie, par continuité et homogénéité, la validité pour tout $\tau \in \mathbf{R}^{N+1}$. Dans le cas où $\operatorname{div}\left(x^{b}\right)$ est effectif, c'est-à-dire quand $b \in \mathbf{N}^{N+1}$, on a $\theta_{\tau, S}(b) \geq \tau_{0} b_{0}+\cdots+\tau_{N} b_{N}$ pour tout $S \in \operatorname{DPC}_{\tau}\left(Q_{\mathcal{A}}\right)$ à cause de la concavité de la toiture du polytope $Q_{\mathcal{A}, \tau}$, et donc

$$
\left(\tau_{0} b_{0}+\cdots+\tau_{N} b_{N}\right) \operatorname{deg}\left(X_{\mathcal{A}}\right) \leq n ! \sum_{S \in \mathrm{DPC}_{\tau}\left(Q_{\mathcal{A}}\right)} \theta_{\tau, S}(b) \operatorname{Vol}_{n}(S),
$$

ainsi

$$
e_{\tau}\left(X_{\mathcal{A}} \cdot \operatorname{div}\left(x^{b}\right)\right) \leq D e_{\tau}\left(X_{\mathcal{A}}\right)-\left(\tau_{0} b_{0}+\cdots+\tau_{N} b_{N}\right) \operatorname{deg}\left(X_{\mathcal{A}}\right) .
$$

Alternativement, on peut démontrer cette inégalité par application directe du théorème 0.2 et de l'exemple IV.1.

On en déduit un théorème de Bézout arithmétique exact pour la hauteur normalisée de l'intersection d'une variété torique avec un diviseur monomial:

Corollaire VII.6. - Soit $K$ un corps de nombres, $\mathcal{A} \in\left(\mathbf{Z}^{n}\right)^{N+1}$ tel que $L_{\mathcal{A}}=\mathbf{Z}^{n}, \alpha \in$ $\left(K^{\times}\right)^{N+1}$ et $b \in \mathbf{Z}^{N+1}$. Posons $\tau_{\alpha v}=\left(\log \left|\alpha_{0}\right|_{v}, \ldots, \log \left|\alpha_{N}\right|_{v}\right)$ pour toute place $v \in M_{K}$ et $D:=\sum_{j=1}^{N} b_{j}$, alors

$$
\begin{aligned}
\widehat{h}\left(X_{\mathcal{A}, \alpha} \cdot \operatorname{div}\left(x^{b}\right)\right) & =D \widehat{h}\left(X_{\mathcal{A}, \alpha}\right)-n ! \sum_{v \in M_{K}} \frac{\left[K_{v}: \mathbf{Q}_{v}\right]}{[K: \mathbf{Q}]} \sum_{S \in \operatorname{DPC}_{\tau_{\alpha} v}\left(Q_{\mathcal{A}}\right)} \theta_{\tau_{\alpha v}, S}(b) \operatorname{Vol}_{n}(S) \\
& =D \widehat{h}\left(X_{\mathcal{A}, \alpha}\right)+\left(\sum_{v \in M_{K}} \frac{\left[K_{v}: \mathbf{Q}_{v}\right]}{[K: \mathbf{Q}]} w_{X_{\mathcal{A}, \tau_{\alpha} v}}\left(x^{b}\right)\right) \operatorname{deg}\left(X_{\mathcal{A}, \alpha}\right) .
\end{aligned}
$$

En particulier, si $\operatorname{div}\left(x^{b}\right)$ est effectif (c'est-à-dire $\left.b \in \mathbf{N}^{N+1}\right)$ on a $\widehat{h}\left(X_{\mathcal{A}, \alpha} \cdot \operatorname{div}\left(x^{b}\right)\right) \leq$ $D \widehat{h}\left(X_{\mathcal{A}, \alpha}\right)$.

Démonstration. - Pour l'identité on remarque que $X_{\mathcal{A}, \alpha} \cdot \operatorname{div}\left(x^{b}\right)=\alpha\left(X_{\mathcal{A}} \cdot \operatorname{div}\left(x^{b}\right)\right)$. En sommant sur $v \in M_{K}$ l'égalité de la proposition VII.5 avec les coefficients $\frac{\left[K_{v}: \mathbf{Q}_{v}\right]}{[K: \mathbf{Q}]}$, on conclut grâce au théorème $\mathbf{V . 1}$

Pour établir l'inégalité, on a par (VII.21)

$$
\begin{aligned}
\widehat{h}\left(X_{\mathcal{A}, \alpha} \cdot \operatorname{div}\left(x^{b}\right)\right) & \leq D \widehat{h}\left(X_{\mathcal{A}, \alpha}\right)-\sum_{v \in M_{K}} \frac{\left[K_{v}: \mathbf{Q}_{v}\right]}{[K: \mathbf{Q}]}\left(\tau_{\alpha v, 0} b_{0}+\cdots+\tau_{\alpha v, N} b_{N}\right) \cdot \operatorname{deg}\left(X_{\mathcal{A}}\right) \\
& \leq D \widehat{h}\left(X_{\mathcal{A}, \alpha}\right)
\end{aligned}
$$

$\operatorname{car} \sum_{v \in M_{K}} \frac{\left[K_{v}: \mathbf{Q}_{v}\right]}{[K: \mathbf{Q}]} \tau_{\alpha v, i}=0$ pour $i=0, \ldots, N$, grâce à la formule du produit.

Si l'on définit la hauteur de $x^{b}$ relative à la variété $X_{\mathcal{A}, \alpha}$ par la formule

$$
\widehat{h}_{X_{\mathcal{A}, \alpha}}\left(x^{b}\right):=\sum_{v \in M_{K}} \frac{\left[K_{v}: \mathbf{Q}_{v}\right]}{[K: \mathbf{Q}]} w_{X_{\mathcal{A}, \tau_{\alpha} v}}\left(x^{b}\right)
$$

le résultat précédent se réécrit

$$
\widehat{h}\left(X_{\mathcal{A}, \alpha} \cdot \operatorname{div}\left(x^{b}\right)\right)=D \widehat{h}\left(X_{\mathcal{A}, \alpha}\right)+\widehat{h}_{X_{\mathcal{A}, \alpha}}\left(x^{b}\right) \cdot \operatorname{deg}\left(X_{\mathcal{A}, \alpha}\right) .
$$




\section{Références}

[AD03] F. Amoroso, S. David, Minoration de la hauteur normalisée dans un tore, J. Inst. Math. Jussieu 2 (2003) 335-381.

[AD04] F. Amoroso, S. David, Le problème de Lehmer en dimension supérieure. II, prépublication de l'université de Caen, 2004.

[Aud91] M. Audin, The topology of torus actions on symplectic manifolds, Progress in Math. 93, Birkhäuser, 1991.

[Ber87] D. Bertrand, Lemmes de zéros et nombres transcendants, Séminaire Bourbaki 1985/86, Astérisque 145 \& 146 (1987) 21-44.

[BP88] D. Bertrand, P. Philippon, Sous-groupes algébriques de groupes algébriques commutatifs, Illinois J. Math. 32 (1988) 263-280.

[Cha89] M. Chardin, Une majoration de la fonction de Hilbert et ses conséquences pour l'interpolation algébrique, Bull. Soc. Math. France 117 (1989) 305-318.

[CP99] M. Chardin, P. Philippon, Régularité et interpolation, J. Algebraic Geom. 8 (1999) 471-481.

[Cox01] D. Cox, Minicourse on toric varieties, notes d'un cours donné à l'université de Buenos Aires en Juillet 2001. Téléchargeable à http//www . amherst. edu/ dacox/.

[CLO98] D. Cox, J. Little, D. O'Shea, Using algebraic geometry, Graduate Texts in Math. 185, Springer, 1998.

[Dav03] S. David, On the height of subvarieties of groups varieties, à paraître dans le J. Ramanujan Math. Soc..

[DP99] S. David, P. Philippon, Minorations des hauteurs normalisées des sous-variétés des tores, Ann. Scuola Norm. Sup. Pisa 28 (1999) 489-543.

[Don02] S.K. Donaldson, Scalar curvature and stability of toric varieties, J. Differential Geom. 62 (2002) 289-349.

[ES96] D. Eisenbud, B. Sturmfels, Binomial ideals, Duke Math. J. 84 (1996) 1-45.

[EF02] J.-H. Evertse, R.G. Ferretti, Diophantine inequalities on projective varieties, Internat. Math. Res. Not. 25 (2002) 1295-1330.

[Ewa96] G. Ewald, Combinatorial convexity and algebraic geometry, Graduate Texts in Math. 168, Springer, 1996.

[Fer03] R.G. Ferretti, Diophantine approximation and toric deformations, Duke Math. J. 118 (2003) 493-522.

[Ful84] W. Fulton, Intersection theory, Ergeb. Math. Grenzgeb. (3) 2, Springer, 1984.

[Ful93] W. Fulton, Introduction to toric varieties, Ann. Math. Studies 131, Princeton Univ. Press, 1993.

[GKZ94] I.M. Gelfand, M.M. Kapranov, A.V. Zelevinsky, Discriminants, resultants and multidimensional determinants, Birkhäuser, 1994.

[KSZ92] M.M. Kapranov, B. Sturmfels, A.V. Zelevinsky, Chow polytopes and general resultants, Duke Math. J. 67 (1992) 189-218.

[Mum77] D. Mumford, Stability of projective varieties, Enseign. Math. 23 (1977) 39-110.

[Phi91] P. Philippon, Sur des hauteurs alternatives, I, Math. Ann. 289 (1991) 255-283.

[PS04] P. Philippon, M. Sombra, Hauteur normalisée des variétés toriques projectives, téléchargeable à http//fr.arxiv.org/abs/math.NT/0406476, 38 pp..

[PS05] P. Philippon, M. Sombra, Géométrie diophantienne et variétés toriques, C. R. Math. Acad. Sci. Paris 340 (2005) 507-512.

[PS06] P. Philippon, M. Sombra, À propos du minimum essentiel des translatés de sous-tores, tapuscrit, 16 pp..

[Rat04] N. Ratazzi, Minoration de la hauteur de Néron-Tate pour les points et les sous-variétés: variations sur le problème de Lehmer, thèse de Doctorat, Université de Paris VI, 2004.

[Rem01a] G. Rémond, Élimination multihomogène, chapitre 5 de Introduction to algebraic independence theory, Lecture Notes in Math. 1752 (2001) 53-81. 
[Rem01b] G. Rémond, Géométrie diophantienne multiprojective, chapitre 7 de Introduction to algebraic independence theory, Lecture Notes in Math. 1752 (2001) 95-131.

[Sch91] W.M. Schmidt, Diophantine approximation and diophantine equations, Lecture Notes in Math. 1467, Springer, 1991.

[Som05] M. Sombra, Minimums successifs des variétés toriques projectives, J. Reine Angew. Math. 586 (2005) 207-233.

[Stu94] B. Sturmfels, On the Newton polytope of the resultant, J. Algebraic Combin. 3 (1994), 207-236.

[Stu96] B. Sturmfels, Gröbner bases and convex polytopes, Amer. Math. Soc., 1996.

[Zha95] S.-W. Zhang, Positive line bundles on arithmetic varieties, J. Amer. Math. Soc. 8 (1995) $187-221$

Patrice Philippon, Institut de Mathématiques de Jussieu (U.M.R. 7586), Projet Géométrie et Dynamique. Case 7012, 2 place Jussieu, 75251 Paris Cedex 05, France.

E-mail : pph@math.jussieu.fr

Url : http://www.math.jussieu.fr/ ${ }^{\sim} p$ ph/

MArtín Sombra, Universitat de Barcelona, Departament d'Àlgebra i Geometria. Gran Via 585, 08007 Barcelona, Espagne.

E-mail : sombra@ub.edu

Url : http://atlas.mat.ub.es/personals/sombra/ 